ANALYSIS \& PDE

Volume $4 \quad$ No. $4 \quad 2011$

\title{
Claudio muñoz C. \\ SOLITON DYNAMICS FOR GENERALIZED KdV EQUATIONS \\ IN A SLOWLY VARYING MEDIUM
}




\title{
SOLITON DYNAMICS FOR GENERALIZED KdV EQUATIONS IN A SLOWLY VARYING MEDIUM
}

\author{
Claudio Muñoz C.
}

\begin{abstract}
We consider the problem of existence and global behavior of solitons for generalized Korteweg-de Vries equations $(\mathrm{gKdV})$ with a slowly varying (in space) perturbation. We prove that such slowly varying media induce on the soliton dynamics large dispersive effects at large times. We also prove that, unlike the unperturbed case, there is no pure-soliton solution to the perturbed $\mathrm{gKdV}$.
\end{abstract}

1. Introduction and main results

2. Preliminaries

3. Construction of a soliton-like solution

4. Description of the soliton-potential interaction 589

$\begin{array}{ll}\text { 5. First stability results } & 598\end{array}$

$\begin{array}{ll}\text { 6. Asymptotics for large times } & 604\end{array}$

7. Proof of the main theorems 616

$\begin{array}{ll}\text { Appendix: Proofs of auxiliary results } & 623\end{array}$

$\begin{array}{ll}\text { Acknowledgments } & 636\end{array}$

$\begin{array}{ll}\text { References } & 636\end{array}$

\section{Introduction and main results}

In this work we consider the following generalized Korteweg-de Vries equation $(\mathrm{gKdV})$ on the real line:

$$
u_{t}+\left(u_{x x}+f(x, u)\right)_{x}=0 \quad \text { in } \mathbb{R}_{t} \times \mathbb{R}_{x} .
$$

Here $u=u(t, x)$ is a real-valued function and $f: \mathbb{R} \times \mathbb{R} \rightarrow \mathbb{R}$ is a nonlinear function. This represents a generalization of the Korteweg-de Vries equation $(\mathrm{KdV})$, which is the case $f(x, s) \equiv s^{2}$ :

$$
u_{t}+\left(u_{x x}+u^{2}\right)_{x}=0 \quad \text { in } \mathbb{R}_{t} \times \mathbb{R}_{x} .
$$

Another physically important case is the cubic one, $f(x, s) \equiv s^{3}$, when (1-1) is often called the (focusing) modified $\mathrm{KdV}$ equation $(\mathrm{mKdV})$, while the case of an arbitrary integer power is what mathematicians generally refer to as the $\mathrm{gKdV}$ :

$$
u_{t}+\left(u_{x x}+u^{m}\right)_{x}=0 \quad \text { in } \mathbb{R}_{t} \times \mathbb{R}_{x} ; \quad m \geq 2 \text { integer. }
$$

The original $\mathrm{KdV}$ equation arises in physics as a model of propagation of dispersive long waves, as

Research supported in part by a CONICYT-Chile and an Allocation de Recherche grants.

MSC2000: primary 35Q51, 35Q53; secondary 37K10, 37K40.

Keywords: generalized $\mathrm{KdV}$ equations, soliton dynamics, slowly varying medium. 
pointed out by J. S. Russel in 1834 [Miura 1976]. The exact formulation of the KdV equation comes from [Korteweg and de Vries 1895]. The equation was rediscovered decades later in a numerical study [Zabusky and Kruskal 1965], after which a great amount of literature — physical, numerical and mathematical has emerged on the subject; see for example [Bona et al. 1980; Kalisch and Bona 2000; Shih 1980; Mizumachi 2003; Miura 1976].

This continuing, focused research on the $\mathrm{KdV}$ (and $\mathrm{gKdV}$ ) equation can be in part explained by some striking algebraic properties. One of the first properties is the existence of localized, exponentially decaying, stable smooth solutions called solitons. For (1-3), solitons are solutions of the form

$$
u(t, x):=Q_{c}\left(x-x_{0}-c t\right), \quad Q_{c}(s):=c^{\frac{1}{m-1}} Q\left(c^{1 / 2} s\right),
$$

where $x_{0}$ and $c>0$ are real numbers and $Q$ is an explicit Schwartz function satisfying the second-order nonlinear differential equation $Q^{\prime \prime}-Q+Q^{m}=0$ :

$$
Q(x)=\left(\frac{m+1}{2 \cosh ^{2}\left(\frac{m-1}{2} x\right)}\right)^{\frac{1}{m-1}} .
$$

This solution represents a "solitary wave" defined for all time moving to the right without any change in shape, velocity, or amplitude.

In addition, Equation (1-3) remains invariant under space and time translations. From Noether's theorem, these symmetries are related to conserved quantities, invariant under the $\mathrm{gKdV}$ flow, usually called mass and energy:

$$
\begin{aligned}
& M[u](t):=\int_{\mathbb{R}} u^{2}(t, x) d x=M[u](0) \\
& E[u](t):=\frac{1}{2} \int_{\mathbb{R}} u_{x}^{2}(t, x) d x-\frac{1}{m+1} \int_{\mathbb{R}} u^{m+1}(t, x) d x=E[u](0) \quad \text { (energy). }
\end{aligned}
$$

We now review some facts about the gKdV equation (1-3), with $m \geq 2$ an integer. The Cauchy problem for Equation (1-1) (that is, the problem with initial condition $u=u_{0}$ at $t=0$ ) is locally well-posed for $u_{0} \in H^{1}(\mathbb{R})$ [Kenig et al. 1993]. In the case $m<5$, any $H^{1}(\mathbb{R})$ solution is global in time, thanks to the conservation equation (1-6), (1-7) and the Gagliardo-Nirenberg inequality

$$
\int_{\mathbb{R}} u^{p+1} \leq K(p)\left(\int_{\mathbb{R}} u^{2}\right)^{\frac{p+3}{4}}\left(\int_{\mathbb{R}} u_{x}^{2}\right)^{\frac{p-1}{4}} .
$$

For $m=5$, solitons are known to be unstable and the Cauchy problem for the corresponding gKdV equation has finite-time blow-up solutions; see [Merle 2001; Martel and Merle 2002b; 2002a] and references therein. It is believed that for $m>5$ the situation is the same. Consequently, in this work, we will discard high-order nonlinearities, at leading order.

In addition, there exists another conservation law, valid only for $L^{1}(\mathbb{R})$ solutions:

$$
\int_{\mathbb{R}} u(t, x) d x=\text { constant. }
$$


The problem to be considered in this paper possesses a long and extensive physical literature. We now briefly describe the main results concerning the propagation of solitons in a slowly varying medium.

Statement of the problem; historical review. The dynamical problem of soliton interaction with a slowly varying medium is by now a classical problem in nonlinear wave propagation. By the soliton-medium interaction we mean, loosely speaking, the following problem: In (1-1), consider a nonlinear function $f=f(t, x, s)$, slowly varying in space and time, possibly of small amplitude, satisfying

$$
f(t, x, s) \sim s^{m} \quad \text { as } x \rightarrow \pm \infty, \quad \text { for all time }
$$

(that is, (1-1) behaves like a gKdV equation at spatial infinity). Consider a soliton solution of the corresponding variable-coefficient equation (1-1) with this nonlinearity, at some early time. We expect that this solution does interact with the medium in space and time, here represented by the nonlinearity $f(t, x, s)$. In a slowly varying medium this interaction, small locally in time, may be important in the long-time behavior of the solution. The resulting solution after the interaction is precisely the object of study. In particular, one considers whether changes in size, position, or shape may be present after some large time, or even the creation or destruction of solitons.

We review some relevant works in this direction. The early works of Fermi, Pasta and Ulam [Fermi et al. 1955] and of Zabusky and Kruskal [1965] established complete integrability for KdV and other equations, leading to a new branch of research devoted to the study of the dynamics of KdV solitons in a slowly varying (in time) medium. (See [Miura 1976] for a review.) In [Kaup and Newell 1978; Karpman and Maslov 1977] the focus is on perturbations of integrable equations, and in particular the perturbed (in time $\tau$ ) gKdV equation

$$
u_{\tau}+\left(\beta(\varepsilon \tau) u_{x x}+\alpha(\varepsilon \tau) u^{m}\right)_{x}=0, \quad m=2,3 ; \quad \alpha, \beta>0 .
$$

This last equation models, for example, the propagation of a wave governed by the KdV equation along a canal of varying depth, among many other physical situations [Karpman and Maslov 1977; Asano 1974].

Note that this equation leaves invariant (1-6) and (1-9), but the corresponding energy for this equation is not conserved anymore. After the transformation

$$
t:=\int_{0}^{\tau} \beta(\varepsilon s) d s, \quad \tilde{u}(t, x):=\left(\frac{\alpha}{\beta}\right)^{\frac{1}{m-1}}(\varepsilon \tau) u(\tau, x),
$$

the preceding equation becomes

$$
\tilde{u}_{t}+\left(\tilde{u}_{x x}+\tilde{u}^{m}\right)_{x}=\varepsilon \gamma(\varepsilon t) \tilde{u}, \quad \text { where } \quad \varepsilon \gamma(\varepsilon t):=\frac{1}{m-1} \partial_{t}\left(\log \left(\frac{\alpha}{\beta}\right) \varepsilon \tau(t)\right) .
$$

The authors performed a perturbative analysis using inverse scattering theory to describe the dynamics of a soliton (for the integrable equation) in this variable regime. Of interest is the existence of a dispersive shelf-like tail behind the soliton, a phenomenon related to the lack of energy conservation (1-7) for the equation (1-11).

The problem was subsequently addressed in several other works and for different integrable models; see, for example, [Ko and Kuehl 1978; Fernandez et al. 1979; Grimshaw 1979a; Grimshaw 1979b]. 
Moreover, using inverse-scattering techniques, the production of a second (and small) solitary wave was pointed out in [Wright 1980] — see also [Grimshaw and Pudjaprasetya 2004] — but a satisfactory analytical proof of this phenomenon is still out of reach. See [Newell 1985, pp. 87-97] for a more detailed account.

Another important motivation comes from Lochak's interesting observation that, based in heuristic conservation laws, well-modulated solitons of (1-11) are good candidates for adiabatically stable objects for this infinite-dimensional dynamical system. See [Lochak 1984; Lochak and Meunier 1988] for details.

In this paper we address the problem of soliton dynamics in the case of an inhomogeneous medium, slowly varying in space but constant in time. This model, from the mathematical point of view, introduces several difficulties, as we will see below; but at the same time it reproduces the creation of a shelf-like tail behind the soliton, as computationally attested by physicists. Our main result is that, as a consequence of this tail, there is no pure soliton solution (unlike gKdV) for this regime. This result illustrates the lack of pure solutions of nontrivial perturbations of $\mathrm{gKdV}$ equations.

Setting and hypotheses. We come back to the general equation (1-1), and consider a small parameter $\varepsilon>0$. Following (1-10), we will assume throughout that the nonlinearity $f$ is a slowly varying $x$-dependent function of the power cases, independent of time, plus a (possibly zero) linear term:

$$
\left\{\begin{array}{l}
f(x, s):=-\lambda s+a_{\varepsilon}(x) s^{m}, \quad \lambda \geq 0, m=2,3 \text { and } 4 . \\
a_{\varepsilon}(x):=a(\varepsilon x) \in C^{3}(\mathbb{R}) .
\end{array}\right.
$$

We will suppose the parameter $\lambda$ fixed and independent of $\varepsilon$. Concerning the function $a$ we will assume that there exist constants $K, \gamma>0$ such that

$$
\begin{cases}1<a(r)<2, \quad a^{\prime}(r)>0 & \text { for } r \in \mathbb{R} \\ 0<a(r)-1 \leq K e^{\gamma r} & \text { for } r \leq 0 \\ 0<2-a(r) \leq K e^{-\gamma r} & \text { for } r \geq 0\end{cases}
$$

Thus

$$
\lim _{r \rightarrow-\infty} a(r)=1 \quad \text { and } \quad \lim _{r \rightarrow+\infty} a(r)=2 ;
$$

however, the special choices (1 and 2) of these limits are irrelevant for the results of this paper. The only necessary conditions are that

$$
0<a_{-\infty}:=\lim _{r \rightarrow-\infty} a(r)<\lim _{r \rightarrow+\infty} a(r)=: a_{\infty}<+\infty .
$$

Finally, to deal with a special stability property of the mass in Theorems 3.1 and 6.1 (see also (6-22)), we will need an additional, but not very restrictive, hypothesis: there exists $K>0$ such that for $m=2,3,4$,

$$
\left|\left(a^{1 / m}\right)^{(3)}(s)\right| \leq K\left(a^{1 / m}\right)^{\prime}(s) \quad \text { for all } s \in \mathbb{R} .
$$

This condition is often satisfied (provided $a^{\prime}$ is not be a compactly supported function). 
Recapitulating, given $0 \leq \lambda<1, \varepsilon_{0}>0$, and a function $a$ satisfying (1-13) and (1-14), we will consider the following equation, for which we use the abbreviation "aKdV" (after the potential $a$ ):

$$
\left\{\begin{array}{l}
u_{t}+\left(u_{x x}-\lambda u+a_{\varepsilon}(x) u^{m}\right)_{x}=0 \quad \text { in } \mathbb{R}_{t} \times \mathbb{R}_{x}, \\
a_{\varepsilon}(x)=a(\varepsilon x), \quad \text { with } m=2,3,4 \text { and } 0<\varepsilon \leq \varepsilon_{0} .
\end{array}\right.
$$

The main issue that we will study in this paper is the interaction problem between a soliton and a slowly varying medium, here represented by the potential $a_{\varepsilon}$. In other words, we intend to study for (1-15) whether it is possible to generalize the well-known soliton-like solution $Q$ of $\mathrm{gKdV}$. It is well-known that in the case $f(t, x, s)=f(s)$, and under reasonable assumptions (see for example [Berestycki and Lions 1983]), there exist soliton-like solutions, constructed via ground states of the corresponding elliptic equation for a bound state. However, in this paper our objective will be the study of soliton solutions for a variable-coefficient equation, where there is no obvious ground state.

As a heuristic introduction to the results to be proved, consider that (1-15) has the form of a $\mathrm{gKdV}$ equation at infinity:

$$
\begin{cases}u_{t}+\left(u_{x x}-\lambda u+1 u^{m}\right)_{x}=0 & \text { as } x \rightarrow-\infty \\ u_{t}+\left(u_{x x}-\lambda u+2 u^{m}\right)_{x}=0 & \text { as } x \rightarrow+\infty .\end{cases}
$$

In particular, if $Q$ is the soliton (1-5) of the standard $\mathrm{gKdV}$ equation, one should be able of to construct a soliton-like solution $u(t)$ of (1-15) such that

$$
u(t) \sim Q(\cdot-(1-\lambda) t) \quad \text { as } t \rightarrow-\infty
$$

in some sense to be defined. Indeed, $Q(\cdot-(1-\lambda) t)$ is an actual solution for the first equation in $(1-16)$ on the whole real line, moving toward the left if $\lambda>1$, toward the right if $\lambda<1$, and stationary if $\lambda=1$.

On the other hand, after passing the interaction region, by stability properties, this solution should behave, for small $\varepsilon$, like

$$
2^{-\frac{1}{m-1}} Q_{c_{\infty}}\left(x-\left(c_{\infty}-\lambda\right) t-\rho(t)\right)+\text { smaller-order terms in } \varepsilon \quad \text { as } t \rightarrow+\infty,
$$

where $c_{\infty}$ is a unknown positive number (a limiting scaling parameter) and $\rho(t)$ is small compared with

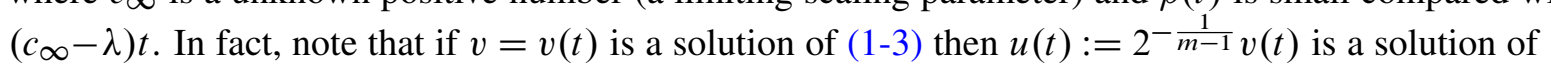

$$
u_{t}+\left(u_{x x}-\lambda u+2 u^{m}\right)_{x}=0 \quad \text { in } \mathbb{R}_{t} \times \mathbb{R}_{x}
$$

In conclusion, this heuristic suggests that even if the potential varies slowly, the soliton will experience nontrivial transformations on its scaling and shape, of the same order as that of the amplitude variation of the potential $a$.

Before we state our results, some important facts are in order. First, Equation (1-15) is unfortunately no longer invariant under scaling and space translations. Moreover, a nonzero solution of (1-15) might lose or gain some mass, depending on the sign of $u$, in the sense that, in the case of rapidly decaying functions, the quantity

$$
M[u](t)=\frac{1}{2} \int_{\mathbb{R}} u^{2}(t, x) d x
$$


satisfies the identity

$$
\partial_{t} M[u](t)=-\frac{\varepsilon}{m+1} \int_{\mathbb{R}} a^{\prime}(\varepsilon x) u^{m+1} .
$$

Another key observation is the following: in the cubic case $m=3$, with our choice of $a_{\varepsilon}$, the mass is always nonincreasing.

On the other hand, when $\lambda \geq 0$, the novel energy

$$
E_{a}[u](t):=\frac{1}{2} \int_{\mathbb{R}} u_{x}^{2}(t, x) d x+\frac{\lambda}{2} \int_{\mathbb{R}} u^{2}(t, x) d x-\frac{1}{m+1} \int_{\mathbb{R}} a_{\varepsilon}(x) u^{m+1}(t, x) d x
$$

remains conserved for all time. Moreover, a simple energy balance at $\pm \infty$ allows one to determine heuristically the limiting scaling in (1-17) in certain cases. For example, if $\lambda=0$, and we suppose that the lower-order terms are of zero mass at infinity, we have from (1-17)

$$
E_{a \equiv 1}[u](-\infty)=E[Q] \sim 2^{-\frac{2}{m-1}} c_{\infty}^{\frac{2}{m-1}+\frac{1}{2}} E[Q]=E_{a \equiv 2}[u](+\infty), \quad E[Q] \neq 0
$$

(see Section A.6 in the Appendix). This implies that $c_{\infty} \sim 2^{\frac{4}{m+3}}>1$. These informal arguments suggest the following definition.

Definition 1.0 (Pure generalized soliton solution for aKdV). Let $0 \leq \lambda<1$ be a fixed number. We will say that (1-15) admits a pure generalized soliton-like solution (of scaling 1) if there exist a $C^{1}$ real valued function $\rho=\rho(t)$ defined for all large times and a global in time $H^{1}(\mathbb{R})$ solution $u(t)$ of (1-15) such that

$$
\lim _{t \rightarrow-\infty}\|u(t)-Q(\cdot-(1-\lambda) t)\|_{H^{1}(\mathbb{R})}=\lim _{t \rightarrow+\infty}\left\|u(t)-2^{-\frac{1}{m-1}} Q_{c_{\infty}}(\cdot-\rho(t))\right\|_{H^{1}(\mathbb{R})}=0,
$$

with $\lim _{t \rightarrow+\infty} \rho(t)=+\infty$, and where $c_{\infty}=c_{\infty}(\lambda)$ is the scaling suggested by the energy conservation law (1-21).

Remark. Note that the existence of a translation parameter $\rho(t)$ is a necessary condition: it is even present in the orbital stability of small perturbations of solitons for $\mathrm{gKdV}$. See [Benjamin 1972; Bona et al. 1987; Cazenave and Lions 1982], for example. We have not included the case $\rho(t) \rightarrow-\infty$ as $t \rightarrow+\infty$, corresponding to a reflected soliton, but we hope to consider this case elsewhere.

Previous analytic results on soliton dynamics in a slowly varying medium. The problem of describing analytically the soliton dynamics of different integrable models in a slowly varying medium has received some increasing attention during the last years. Concerning the $\mathrm{KdV}$ equation, our belief is that the first result in this direction was given in [Dejak and Jonsson 2006; Dejak and Sigal 2006]. These works considered the long time dynamics of solitary waves (solitons) over slowly varying perturbations of $\mathrm{KdV}$ and modified $\mathrm{KdV}$ equations

$$
u_{t}+\left(u_{x x}-b(t, x) u+u^{m}\right)_{x}=0 \quad \text { on } \mathbb{R}_{t} \times \mathbb{R}_{x}, \quad m=2,3,
$$

and where $b$ is assumed having small size and small variation, in the sense that for $\varepsilon$ small,

$$
\left|\partial_{t}^{n} \partial_{x}^{p} b\right| \leq \varepsilon^{n+p+1} \quad \text { for } 0 \leq n+p \leq 2 .
$$


(Actually their conclusions hold in more generality, but for our purposes we state the closest version to our approach; see [Dejak and Jonsson 2006] for the detailed version.) With these hypotheses the authors showed that if $m=2$ and the initial condition $u_{0}$ satisfies the orbital stability condition

$$
\inf _{\substack{0<c_{0}<c<c_{1} \\ a \in \mathbb{R}}}\left\|u_{0}-Q_{c}(\cdot-a)\right\|_{H^{1}(\mathbb{R})} \leq \varepsilon^{2 s}, \quad s<\frac{1}{2}, c_{0}, c_{1} \text { given, }
$$

then for any for time $t \leq K \varepsilon^{-s}$ the solution can be decomposed as

$$
u(t, x)=Q_{c(t)}(x-\rho(t))+w(t, x),
$$

where $\|w(t)\|_{H^{1}(\mathbb{R})} \leq K \varepsilon^{s}$ and $\rho(t), c(t)$ satisfies the following differential system

$$
\rho^{\prime}(t)=c(t)-b(t, a(t))+O\left(\varepsilon^{2 s}\right), \quad c^{\prime}(t)=O\left(\varepsilon^{2 s}\right) ;
$$

during the interval of time considered. In the cubic case $(m=3)$ the results are slightly better; see [Dejak and Jonsson 2006].

Our model can be written as a generalized, time-independent Dejak-Jonsson-Sigal equation of the type (1-22), after writing $v(t, x):=\tilde{a}(\varepsilon x) u(t, x)$, with $\tilde{a}(\varepsilon x):=a^{\frac{1}{m-1}}(\varepsilon x)$. From these considerations we expect to recover and to improve the results obtained by those authors.

Holmer [ $\geq 2011]$ has announced some improvements on the Dejak-Sigal results, by assuming $b$ of amplitude $O_{L^{\infty}}(1)$. He proves that

$$
\sup _{t \lesssim \delta \varepsilon^{-1}|\log \varepsilon|}\|w(t)\|_{H^{1}(\mathbb{R})} \lesssim \varepsilon^{1 / 2-\delta}, \quad \text { for some } \delta>0 .
$$

In this paper we have preferred to avoid the inclusion of a time-dependent potential, and to treat the infinite time prescribed and pure data, instead of the standard Cauchy problem. This choice will have positive consequences for our main results, Theorems 1.1 and 1.2, where we will describe with accuracy the dynamical problem, including its asymptotics as $t \rightarrow+\infty$.

The soliton-potential interaction can be considered also in the case of the nonlinear Schrödinger equation

$$
i u_{t}+u_{x x}-V(\varepsilon x) u+|u|^{2} u=0 \quad \text { on } \mathbb{R}_{t} \times \mathbb{R}_{x} ;
$$

see [Muñoz $\geq 2011 \mathrm{~b}$ ], for example. Results similar to the ones just mentioned were obtained in [Holmer and Zworski 2008; Holmer et al. 2007a; 2007b; Jonsson et al. 2006; Fröhlich et al. 2004]. Finally we point out the recent [Perelman 2009], concerning the critical quintic NLS equation.

Main results. Let

$$
T_{\varepsilon}:=\frac{1}{1-\lambda} \varepsilon^{-1-\frac{1}{100}}>0 .
$$

This parameter can be understood as the interaction time between the soliton and the potential. In other words, at time $t=-T_{\varepsilon}$ the soliton should remain almost unperturbed, and at time $t=T_{\varepsilon}$ the soliton should have completely crossed the influence region of the potential. Note that the asymptotic $\lambda \sim 1$ is a degenerate case and it will not be considered in this work. 
In Theorems 1.1, 1.2, and 1.3 we will show that, under suitable assumptions, a pure soliton-like solution as in Definition 1.0 does not exist, in the sense that the lower order terms appearing after the interaction always have positive mass. This phenomenon will be a consequence of the dispersion produced during the crossing of the soliton with the main core of the potential $a_{\varepsilon}$.

We will from now on assume the validity of assumptions (1-12), (1-13), and (1-14). Our first result describes the dynamics of the pure soliton-like solution for the aKdV equation (1-15).

Theorem 1.1 (Dynamics of interaction of solitons for $\mathrm{gKdV}$ equations in a variable medium). Let $m=2,3,4$, and let $0 \leq \lambda \leq \lambda_{0}:=\frac{5-m}{m+3}$ be a fixed number. There exists a small constant $\varepsilon_{0}>0$ such that for all $0<\varepsilon<\varepsilon_{0}$ the following statements hold.

(1) Existence of a soliton-like solution. There exists a solution $u \in C\left(\mathbb{R}, H^{1}(\mathbb{R})\right)$ of $(1-15)$, global in time, such that

$$
\lim _{t \rightarrow-\infty}\|u(t)-Q(\cdot-(1-\lambda) t)\|_{H^{1}(\mathbb{R})}=0,
$$

with conserved energy $E_{a}[u](t)=\left(\lambda-\lambda_{0}\right) M[Q] \leq 0$. This solution is unique if $m=3$, or if $m=2,4$ and $\lambda>0$.

(2) Soliton-potential interaction. There exist $K>0$ and numbers $c_{\infty}(\lambda) \geq 1, \rho_{\varepsilon}, \tilde{T}_{\varepsilon} \in \mathbb{R}$ such that the solution $u(t)$ above satisfies

$$
\left\|u\left(\tilde{T}_{\varepsilon}\right)-2^{-1 /(m-1)} Q_{c_{\infty}}\left(x-\rho_{\varepsilon}\right)\right\|_{H^{1}(\mathbb{R})} \leq K \varepsilon^{1 / 2} .
$$

Moreover,

$$
c_{\infty}(\lambda=0)=2^{\frac{4}{m+3}} \quad \text { and } \quad c_{\infty}\left(\lambda=\lambda_{0}\right)=1
$$

Finally we have the bounds

$$
\left|T_{\varepsilon}-\tilde{T}_{\varepsilon}\right| \leq \frac{T_{\varepsilon}}{100} \quad \text { and } \quad(1-\lambda) T_{\varepsilon} \leq \rho_{\varepsilon} \leq\left(2 c_{\infty}(\lambda)-\lambda-1\right) T_{\varepsilon}
$$

Note that $\lambda_{0}=\lambda_{0}(m)$ is always less than 1 for $m=2,3,4$, while $\lambda_{0}=0$ for $m=5$ (the $L^{2}$-critical case). Also, for $\lambda=\lambda_{0}$ we have $E_{a}[u](t)=\left(\lambda-\lambda_{0}\right) M[Q]=0$; and if $\lambda<\lambda_{0}$ we have $E_{a}[u](t)<0$ for all $t \in \mathbb{R}$. For the consequences of this property and a detailed study of $c_{\infty}(\lambda)$, see Lemma 4.4.

Remark. The proof of Theorem 1.1 is based on the construction of an approximate solution of (1-15) in the interaction region, satisfying certain symmetries. However, at some point we formally obtain an infinite mass term (see [Martel and Merle 2011; 2010] for a similar problem). It turns out that to obtain a localized solution we need to break the symmetry of this solution (see Proposition 4.7 for the details). This lack of symmetry leads to the error $\varepsilon^{1 / 2}$ in the theorem. At this price we describe completely the interaction, a completely new result.

The next step is understanding the long time behavior of our generalized soliton solution.

Theorem 1.2 (Long time behavior). Suppose, in addition to the assumptions of Theorem 1.1, that $0<\lambda \leq \lambda_{0}$ for the cases $m=2,4$, and $0 \leq \lambda \leq \lambda_{0}$ if $m=3$. Let $0<\beta<\frac{1}{2}\left(c_{\infty}(\lambda)-\lambda\right)$. There exists a 
constant $\varepsilon_{0}>0$ such that, for all $0<\varepsilon \leq \varepsilon_{0}$, there exist $K, c^{+}>0$ and a $C^{1}$-function $\rho_{2}(t)$ defined in $\left[T_{\varepsilon},+\infty\right)$ such that the function

$$
w^{+}(t, \cdot):=u(t, \cdot)-2^{\frac{-1}{m-1}} Q_{c^{+}}\left(\cdot-\rho_{2}(t)\right)
$$

has the following properties:

(1) Stability. For any $t \geq T_{\varepsilon}$,

$$
\left\|w^{+}(t)\right\|_{H^{1}(\mathbb{R})}+\left|c^{+}-c_{\infty}(\lambda)\right|+\left|\rho_{2}^{\prime}(t)-\left(c_{\infty}(\lambda)-\lambda\right)\right| \leq K \varepsilon^{1 / 2} .
$$

(2) Asymptotic stability.

$$
\lim _{t \rightarrow+\infty}\left\|w^{+}(t)\right\|_{H^{1}(x>\beta t)}=0 .
$$

(3) Bounds on the parameters. Define $\theta:=\frac{1}{m-1}-\frac{1}{4}>0$. The limit

$$
\lim _{t \rightarrow+\infty} E_{a}\left[w^{+}\right](t)=: E^{+}
$$

exists and satisfies the identity

$$
E^{+}=\frac{\left(c^{+}\right)^{2 \theta}}{2^{2 /(m-1)}}\left(\lambda_{0} c^{+}-\lambda\right) M[Q]+\left(\lambda-\lambda_{0}\right) M[Q],
$$

and for all $m=2,3,4$ and $0<\lambda \leq \lambda_{0}$ there exists $K(\lambda)>0$ such that

$$
\frac{1}{K} \limsup _{t \rightarrow+\infty}\left\|w^{+}(t)\right\|_{H^{1}(\mathbb{R})}^{2} \leq E^{+} \leq K \varepsilon \text {. }
$$

In the case $m=3$, we have $\frac{3}{2} E^{+}=\left(\frac{c^{+}}{c_{\infty}}\right)^{3 / 2}-1$ if $\lambda=0$, and

$$
\frac{1}{K} \limsup _{t \rightarrow+\infty}\left\|w^{+}(t)\right\|_{H^{1}(\mathbb{R})}^{2} \leq\left(\frac{c^{+}}{c_{\infty}}\right)^{2 \theta}-1 \leq K \varepsilon \quad \text { if } \lambda>0 .
$$

Remarks. (a) The stability and asymptotic stability of solitary waves for generalized KdV equations have been widely studied since the 1980s. The main ideas in our proof of (1-29) and (1-30) appear in the literature; see [Benjamin 1972; Cazenave and Lions 1982; Bona et al. 1987; Martel et al. 2002; Pego and Weinstein 1994], for example.

(b) The sign of $a^{\prime}(\cdot)$ is a sufficient condition to ensure stability, but it is conceivable that it can be replaced by a weaker one, say $a^{\prime}(s)>0$ for all $s>s_{0}$.

Changes for decreasing potentials. Suppose the potential $a(\cdot)$ satisfies instead $a^{\prime}(s)<0$ and

$$
1=\lim _{s \rightarrow-\infty} a(s)>a(s)>\lim _{t \rightarrow+\infty} a(s)=\frac{1}{2} .
$$

Statement (1) of Theorem 1.1 remains true, except that we do not know whether the solution is unique. Part (2) holds true with the coefficient $2^{\frac{1}{m-1}}$ in front of $Q_{c_{\infty}}, \frac{\lambda}{\lambda_{0}}<c_{\infty}(\lambda)<1$, and $c_{\infty}=2^{-p}$ for $\lambda=0$ (see Lemma 4.4 for this). Bounds similar to (1-28) hold true, with the obvious changes. By contrast, we have no analog for Theorem 1.2: long-time stability for decreasing potentials remains an open question. 
A fundamental question arises from Theorems 1.1 and 1.2: Is the solution a pure soliton (Definition 1.0) for the aKdV equation with $a_{\varepsilon} \equiv 2$ ? This question is equivalent to deciding whether

$$
\limsup _{t \rightarrow+\infty}\left\|w^{+}(t)\right\|_{H^{1}(\mathbb{R})}=0 .
$$

Our last result shows that this behavior cannot happen.

Theorem 1.3 (Nonexistence of pure soliton-like solutions for $\mathrm{aKdV}$ ). With the assumptions and notation of Theorems 1.1 and 1.2, suppose in addition that $m=2,3,4$ with $0<\lambda \leq \lambda_{0}$. There exists $\varepsilon_{0}>0$ such that, for all $0<\varepsilon<\varepsilon_{0}$,

$$
\limsup _{t \rightarrow+\infty}\left\|w^{+}(t)\right\|_{H^{1}(\mathbb{R})}>0 .
$$

Remark. In addition to the classical problem of extending the results to more general potentials $a(\cdot)$, several related questions arise naturally, which we are as yet unable to solve:

(1) Is every solution of (1-15) with $H^{1}(\mathbb{R})$ data globally bounded in time? In Proposition 2.2 we prove that every solution is globally well defined for all positive times, and uniformly bounded if $\lambda>0$ or $m=3$. However, for the cases $m=2,4$ and $\lambda=0$ we only have been able to find an exponential upper bound on the $H^{1}$-norm of the solution. Is every solution described in Theorem 1.1 globally bounded?

(2) In the cases $m=2,4$ and $\lambda=0$, is the solution constructed in Theorem 1.1 unique? Is it stable for large times? (Compare Theorem 6.1.)

(3) What is the behavior of the solution for a coefficient $\lambda_{0}<\lambda<1$ ? We believe in this situation the soliton still survives, but is reflected by the potential, propagating to the left for large $t$. (See [Muñoz $\geq 2011 \mathrm{a}]$.)

(4) [Added in proof] We have recently proved a quantitative lower bound on the defect [Muñoz 2011].

(5) Is there scattering modulo the soliton solution, at infinity?

The case of a time-dependent potential. As might be expected, our results are also valid, with easier proofs, for the time-dependent $\mathrm{gKdV}$ equation

$$
u_{t}+\left(u_{x x}-\lambda u+a(\varepsilon t) u^{m}\right)_{x}=0 \quad \text { in } \mathbb{R}_{t} \times \mathbb{R}_{x},
$$

where $a$ satisfies (1-13)-(1-14), with the time variable in place of $r$. This equation is invariant under scaling and space translations. In addition, the $L^{1}$ integral and the mass $M[u]$ remain constants and the energy

$$
\tilde{E}[u](t):=\frac{1}{2} \int_{\mathbb{R}} u_{x}^{2}+\frac{\lambda}{2} \int_{\mathbb{R}} u^{2}-\frac{a(\varepsilon t)}{m+1} \int_{\mathbb{R}} u^{m+1}
$$

satisfies

$$
\partial_{t} \tilde{E}[u](t)=-\frac{\varepsilon a^{\prime}(\varepsilon t)}{m+1} \int_{\mathbb{R}} u^{m+1} .
$$

Theorems 1.1 and 1.2 hold with $c_{\infty}(\lambda=0)=2^{4 /(5-m)}$ (because of mass conservation), for any $\lambda \geq 0$ and $m=2,3,4$ (follow Lemma 4.4 to see this). We leave the details to the reader. 
Sketch of proofs. Our arguments combine techniques adapted from [Martel 2005; Martel et al. 2010; Martel and Merle 2008; 2011; 2007; 2010] with some new computations. We separate the analysis into three time intervals: $t \ll-\varepsilon^{-1},|t| \leq \varepsilon$, and $\varepsilon^{-1} \ll t$. On each interval the solution possesses a specific behavior:

$t \ll-\varepsilon^{-1}$ : In this interval of time we prove that $u(t)$ remains very close to a soliton solution, with no change in the scaling and shift parameters (Theorem 3.1). This is possible for very large negative times, where the soliton is still far from the interacting region $|t| \leq \varepsilon^{-1}$.

$|t| \leq \varepsilon^{-1}$ : Here the soliton-potential interaction leads the dynamics of $u(t)$. The novelty here is the construction of an approximate solution of (1-15) with high order of accuracy such that: (a) at time $t \sim-\varepsilon^{-1}$ this solution is close to the soliton solution and therefore to $u(t)$; (b) it describes the soliton-potential interaction inside this interval, in particular we show the existence of a dispersive tail behind the soliton; and (c) it is close to $u(t)$ in the whole interval $\left[-\varepsilon^{-1}, \varepsilon^{-1}\right]$, uniformly on time, apart from a modulation on a translation parameter (Theorem 4.1).

$t \gg \varepsilon^{-1}$ : Here some stability properties (Theorem 6.1) are used to establish the convergence of the solution $u(t)$ to a soliton-like solution with modified parameters.

Additionally, by using a contradiction argument, it will be possible to show that the residue of the interaction at time $t \sim \varepsilon^{-1}$ is still present at infinity. This gives the conclusion of the main Theorems 1.1 and 1.3. Indeed, recall the $L^{1}$ conserved quantity from (1-9). This expression is in general useless when the equation is considered on the whole real line $\mathbb{R}$, but it has some striking applications in the blow-up theory (see [Merle 2001]). In our case, it will be useful in discarding the existence of a pure soliton-like solution.

Accordingly, the paper is organized as follows. In Section 2 we introduce some basic tools to study the interaction and asymptotic problems. Section 3 is devoted to the construction of the soliton like solution for large negative time. Sections 4 and 5 deal with the proof of Theorem 1.1. In Section 6 we prove the asymptotic behavior as $t \rightarrow+\infty$, namely Theorem 1.2. Finally we prove Theorem 1.3 (Section 7).

Remark. We believe that the main results of this paper are also valid for general subcritical nonlinearities, with stable solitons. In this case the scaling property of the soliton is no longer valid, so in order to construct an approximate solution one should modify the main argument of the proof.

\section{Preliminaries}

Throughout this paper, $C, K$, and $\gamma>0$ will denote constants independent of $\varepsilon$, possibly changing from one line to another.

To treat the case $\lambda>0$ we need to extend the energy (1-7) by adding a mass term. We therefore introduce a new energy function $E_{1}[u]$, the particular case of (1-21) when $a \equiv 1$.

The Cauchy problem. First we develop a suitable local well-posedness theory for the Cauchy problem associated to (1-15). 
Let $u_{0} \in H^{s}(\mathbb{R}), s \geq 1, \lambda \geq 0$. We consider the initial value problem

$$
\left\{\begin{array}{l}
u_{t}+\left(u_{x x}-\lambda u+a_{\varepsilon}(x) u^{m}\right)_{x}=0 \quad \text { in } \mathbb{R}_{t} \times \mathbb{R}_{x} \\
u(t=0)=u_{0},
\end{array}\right.
$$

where $m=2,3,4$. The analogous problem for the standard $\mathrm{gKdV}$ equation (1-3) has been extensively studied. For dealing with (2-1), we will follow closely the contraction method developed in [Kenig et al. 1993]. The following result is proved with standard techniques based on the Picard iteration procedure and the tools developed in this last reference:

Proposition 2.1 (Local well-posedness in $H^{s}(\mathbb{R})$ ). (See also [Kenig et al. 1993]). Suppose $u_{0} \in H^{s}(\mathbb{R})$, $s \geq 1$. Then (2-1) has a unique (in a certain sense) solution $u \in C\left(I, H^{s}(\mathbb{R})\right)$ defined in a maximal interval of existence $I \ni 0$. Moreover:

(1) Blow-up alternative. If $\sup I<+\infty$, then

$$
\lim _{t \uparrow \sup I}\|u(t)\|_{H^{s}(\mathbb{R})}=+\infty .
$$

The same conclusion holds if inf $I>-\infty$.

(2) Energy conservation. For any $t \in I$ the energy $E_{a}[u](t)$ from (1-21) remains constant.

(3) Mass variation. For all $t \in I$ the mass $M[u](t)$ defined in (1-19) satisfies (1-20).

(4) Suppose $u_{0} \in L^{1}(\mathbb{R}) \cap H^{1}(\mathbb{R})$. Then (1-9) is well defined and remains constant for all $t \in I$.

Once local-in-time existence is established, the next step is to ask for the possibility of a global well-posedness theorem. In many cases the proof reduces to the use of conservation laws to obtain bounds on the norm of the solution for every time. In the case of $\mathrm{gKdV}$ equations $(m \leq 4)$ this was proved in [Kenig et al. 1993] using mass and energy conservation; however, in our case (1-20) is not enough to control the $L^{2}$ norm of the solution. As stated in the Introduction, global existence for cubic case $m=3$ follows from the mass decreasing property. However, to deal with the remaining cases, we will modify our arguments by introducing a perturbed mass, almost decreasing in time, in order to prove global existence. Indeed, define for each $t \in I$ and $m=2,3,4$ the quantity

$$
\hat{M}[u](t):=\frac{1}{2} \int_{\mathbb{R}} a_{\varepsilon}^{1 / m}(x) u^{2}(t, x) d x .
$$

It is clear that $\hat{M}[u](t)$ is well defined, for any time $t \in I$ and solution $u \in H^{1}(\mathbb{R})$ of (2-1). For all $t \in I$ we have the equivalence property

$$
M[u](t) \leq \hat{M}[u](t) \leq 2^{1 / m} M[u](t) .
$$

This modified mass enjoys a striking property:

Proposition 2.2 (Global existence in $H^{1}(\mathbb{R})$ ). Consider the solution $u(t)$ of the Cauchy problem $(2-1)$ with $u(0)=u_{0} \in H^{1}(\mathbb{R})$ and maximal interval of existence $I$. Then $u(t)$ is continuously well defined in $H^{1}(\mathbb{R})$ for any $t \geq 0$. More precisely: 
(1) Cubic case. Suppose $m=3, \lambda \geq 0$. Then $I=\left(\tilde{t}_{0},+\infty\right)$ for some $-\infty \leq \tilde{t}_{0}<0$ and there exists $K=K\left(\left\|u_{0}\right\|_{H^{1}(\mathbb{R})}\right)>0$ such that

$$
\sup _{t \geq 0}\|u(t)\|_{H^{1}(\mathbb{R})} \leq K
$$

(2) Almost monotonicity of the modified mass $\hat{M}$ and global existence. For any $m=2,3,4$ and for all $t \in I$ we have

$$
\partial_{t} \hat{M}[u](t)=-\frac{3}{2} \varepsilon \int_{\mathbb{R}}\left(a^{1 / m}\right)^{\prime}(\varepsilon x) u_{x}^{2}-\frac{\varepsilon}{2} \int_{\mathbb{R}}\left[\lambda\left(a^{1 / m}\right)^{\prime}-\varepsilon^{2}\left(a^{1 / m}\right)^{(3)}\right](\varepsilon x) u^{2} .
$$

In particular, (a) I is again of the form $\left(\tilde{t}_{0},+\infty\right)$; (b) item if $\lambda>0$ there exists $\varepsilon_{0}>0$ small such that (2-5) holds; and (c) if $\lambda=0$ and $m=2,4$, there exists $K=K\left(\left\|u_{0}\right\|_{H^{1}(\mathbb{R})}\right)$ such that we have for all $t \geq 0$ the exponential bound

$$
\|u(t)\|_{H^{1}(\mathbb{R})} \leq K e^{K \varepsilon^{3} t} .
$$

Proof of Proposition 2.2. First we consider the cubic case, $m=3$. From (1-20) we have

$$
M[u](t) \leq M[u](0) \text { for any } t \in I, t \geq 0 .
$$

This bound implies global existence for positive times. Indeed, the bound rules out a $L^{2}$ blow-up in (positive) finite and infinite time, namely (2-2). In order to control the $H^{1}(\mathbb{R})$ norm, we use energy conservation, the Gagliardo-Nirenberg inequality (1-8), and the preceding bound on the mass. Indeed, for any $t \in I, t \geq 0$, and redefining the constant $K$ if necessary, we have

$$
\begin{aligned}
\frac{1}{2} \int_{\mathbb{R}} u_{x}^{2} & =E_{a}[u](0)-\frac{1}{2} \lambda \int_{\mathbb{R}} u^{2}+\frac{1}{m+1} \int_{\mathbb{R}} a_{\varepsilon} u^{m+1} \\
& \leq E_{a}[u](0)+\lambda M[u](0)+K\|u(t)\|_{L^{2}(\mathbb{R})}^{(m+3) / 2}\left\|u_{x}(t)\right\|_{L^{2}(\mathbb{R})}^{(m-1) / 2}
\end{aligned}
$$

Noticing that $\frac{1}{4}(m-1)<1$ for $m=2,3,4$, we have

$$
\int_{\mathbb{R}} u_{x}^{2} \leq K\left(\lambda,\left\|u_{0}\right\|_{H^{1}(\mathbb{R})}\right)
$$

for a large constant $K$. This implies the $H^{1}(\mathbb{R})$ global existence for all positive times and the uniform bound in time (2-5).

To prove (2-6), we proceed by formally taking the time derivative. Every step can be rigorously justified by introducing mollifiers. From (1-15) we have

$$
\begin{aligned}
\partial_{t} \hat{M}[u](t)= & \int_{\mathbb{R}} a_{\varepsilon}^{1 / m} u u_{t}=\int_{\mathbb{R}}\left(a_{\varepsilon}^{1 / m} u\right)_{x}\left(u_{x x}-\lambda u+a_{\varepsilon} u^{m}\right) \\
= & \varepsilon \int_{\mathbb{R}}\left(\left(a^{1 / m}\right)^{\prime}(\varepsilon x) u u_{x x}-\frac{1}{2}\left(a^{1 / m}\right)^{\prime}(\varepsilon x) u_{x}^{2}\right)-\frac{\lambda}{2} \varepsilon \int_{\mathbb{R}}\left(a^{1 / m}\right)^{\prime}(\varepsilon x) u^{2} \\
& \quad+\varepsilon \int_{\mathbb{R}} a_{\varepsilon}\left(a^{1 / m}\right)^{\prime}(\varepsilon x) u^{m+1}-\frac{\varepsilon}{m+1} \int_{\mathbb{R}}\left(a^{1 / m+1}\right)^{\prime}(\varepsilon x) u^{m+1} \\
= & -\frac{1}{2} \varepsilon \int_{\mathbb{R}}\left[\lambda\left(a^{1 / m}\right)^{\prime}(\varepsilon x)-\varepsilon^{2}\left(a^{1 / m}\right)^{(3)}(\varepsilon x)\right] u^{2}-\frac{3}{2} \varepsilon \int_{\mathbb{R}}\left(a^{1 / m}\right)^{\prime}(\varepsilon x) u_{x}^{2}
\end{aligned}
$$


This proves (2-6). Now, in order to establish global $H^{1}(\mathbb{R})$ existence for positive times, we first control the $L^{2}$ norm using $\hat{M}[u](t)$. Let us consider the case $\lambda>0$. In this case, taking $\varepsilon_{0}$ small enough, and thanks to (1-14), we have

$$
\partial_{t} \hat{M}[u](t) \leq 0
$$

and thus $\hat{M}[u](t) \leq \hat{M}[u](0)$ for all $t \in I, t \geq 0$. The rest of the proof is identical to the cubic case.

Now we consider the last case, namely $m=2,4$ and $\lambda=0$. Here the argument above is not valid anymore; we have only the existence of $K>0$ independent of $\varepsilon$ such that

$$
\partial_{t} \hat{M}[u](t) \leq K \varepsilon^{3} \hat{M}[u](t)
$$

This implies that, for any $t \in I$ with $t \geq 0$,

$$
M[u](t) \leq \hat{M}[u](t) \leq K \hat{M}[u](0) e^{K \varepsilon^{3} t} .
$$

This bound rules out a $L^{2}$ blow-up in finite time for positive times. To control the $H^{1}(\mathbb{R})$ norm, we use the same argument from the preceding case. Indeed, for any $t \in I$, redefining the constant $K$ if necessary, we have

$$
\int_{\mathbb{R}} u_{x}^{2} \leq K e^{K \varepsilon^{3} t}
$$

This implies the global $H^{1}(\mathbb{R})$ existence for positive times.

Remark (Mass monotonicity). Consider a solution $u(t) \in H^{1}(\mathbb{R})$ of (1-15) and define the modified mass

$$
\tilde{M}[u](t):= \begin{cases}M[u](t) & \text { if } m=3, \\ \hat{M}[u](t) & \text { if } m=2,4 \text { and } \lambda>0 .\end{cases}
$$

Proposition 2.2 implies that there exists $\varepsilon_{0}>0$ such that $\tilde{M}[u](t)-\tilde{M}[u]\left(t_{0}\right) \leq 0$, for all $0<\varepsilon \leq \varepsilon_{0}$ and all $t \in \mathbb{R}$ with $t \geq t_{0}$.

Spectral properties of the linearized $\mathbf{g K d V}$ operator. We next consider some important properties of the linearized operator associated to (1-15). Fix $c>0$ and $m=2,3,4$, and let

$$
\mathscr{L} w(y):=-w_{y y}+c w-m Q_{c}^{m-1}(y) w, \quad \text { where } \quad Q_{c}(y):=c^{\frac{1}{m-1}} Q(\sqrt{c} y) .
$$

Here $w=w(y)$. We also denote $\mathscr{L}_{0}:=\mathscr{L}_{c=1}$.

Lemma 2.3 (Spectral properties of $\mathscr{L}$ ). (See [Martel and Merle 2009].) The operator $\mathscr{L}$ defined on $L^{2}(\mathbb{R})$ by (2-9) has domain $H^{2}(\mathbb{R})$, is self-adjoint, and satisfies the following properties:

(1) First eigenvalue. There exists a unique $\lambda_{m}>0$ such that $\mathscr{L} Q_{c}^{\frac{m+1}{2}}=-\lambda_{m} Q_{c}^{\frac{m+1}{2}}$.

(2) The kernel of $\mathscr{L}$ is spanned by $Q_{c}^{\prime}$, and we have $\mathscr{L}\left(\Lambda Q_{c}\right)=-Q_{c}$, where

$$
\Lambda Q_{c}:=\left.\partial_{c^{\prime}} Q_{c^{\prime}}\right|_{c^{\prime}=c}=\frac{1}{c}\left(\frac{1}{m-1} Q_{c}+\frac{1}{2} x Q_{c}^{\prime}\right) .
$$

The continuous spectrum of $\mathscr{L}$ is given by $\sigma_{\text {cont }}(\mathscr{L})=[c,+\infty)$.

(3) Inverse. For all $h \in L^{2}(\mathbb{R})$ such that $\int_{\mathbb{R}} h Q_{c}^{\prime}=0$, there exists a unique $\hat{h} \in H^{2}(\mathbb{R})$ such that $\int_{\mathbb{R}} \hat{h} Q_{c}^{\prime}=0$ and $\mathscr{L} \hat{h}=h$. Moreover, if $h$ is even (resp. odd), then $\hat{h}$ is even (resp. odd). 
(4) Regularity in the Schwartz space $\mathscr{Y}$. For $h \in H^{2}(\mathbb{R}), \mathscr{L} h \in \mathscr{Y}$ implies $h \in \mathscr{Y}$.

(5) Coercivity.

(a) There exist $K, \sigma_{c}>0$ such, that for all $w \in H^{1}(\mathbb{R})$,

$$
\mathscr{B}[w, w]:=\int_{\mathbb{R}}\left(w_{x}^{2}+c w^{2}-m Q_{c}^{m-1} w^{2}\right) \geq \sigma_{c} \int_{\mathbb{R}} w^{2}-K\left|\int_{\mathbb{R}} w Q_{c}\right|^{2}-K\left|\int_{\mathbb{R}} w Q_{c}^{\prime}\right|^{2} .
$$

In particular, if $\int_{\mathbb{R}} w Q_{c}=\int_{\mathbb{R}} w Q_{c}^{\prime}=0$, then the functional $\mathscr{B}[w, w]$ is positive definite in $H^{1}(\mathbb{R})$.

(b) The same conclusion holds if $\int_{\mathbb{R}} w Q_{c}=\int_{\mathbb{R}} w x Q_{c}=0$,.

Now we introduce some notation, taken from [Martel and Merle 2007]. We denote by 9 the set of $C^{\infty}$ functions $f$ such that for all $j \in \mathbb{N}$ there exist $K_{j}, r_{j}>0$ such that for all $x \in \mathbb{R}$ we have

$$
\left|f^{(j)}(x)\right| \leq K_{j}(1+|x|)^{r_{j}} e^{-|x|} .
$$

Now we recall a function used to describe the effect of dispersion on the solution. Set $\varphi(x):=-\frac{Q^{\prime}(x)}{Q(x)}$; then $\varphi$ is an odd function and has the following properties (see [Martel and Merle 2009]):

(1) $\lim _{x \rightarrow-\infty} \varphi(x)=-1$ and $\lim _{x \rightarrow+\infty} \varphi(x)=1$.

(2) For all $x \in \mathbb{R}$, we have $\left|\varphi^{\prime}(x)\right|+\left|\varphi^{\prime \prime}(x)\right|+\left|\varphi^{(3)}(x)\right| \leq C e^{-|x|}$.

(3) $\varphi^{\prime} \in \mathcal{Y}$ and $1-\varphi^{2} \in \mathcal{Y}$.

For $c>0$, we then set

$$
\varphi_{c}(x):=-\frac{Q_{c}^{\prime}}{Q_{c}}=\sqrt{c} \varphi(\sqrt{c} x) .
$$

Remark. The same function $\varphi$ has been used in [Martel and Merle 2007] to describe the main-order nonlinearity effect on the phase of two colliding solitons for the quartic $\mathrm{KdV}$ equation. Here $\varphi$ will describe the dispersive tail behind the soliton produced by the interaction with the potential $a_{\varepsilon}$. For details, see Lemma 4.3.

We conclude the section with a result taken from [Martel and Merle 2007].

Lemma 2.4 (Nontrivial kernel). There exists a unique even solution of the problem

$$
\mathscr{L}_{0} V_{0}=m Q^{m-1}, \quad V_{0} \in \mathscr{Y} .
$$

This solution satisfies

$$
\left(\mathscr{L}_{0}\left(1+V_{0}\right)\right)^{\prime}=0
$$

and it is given, in the notation of Lemma 2.3, by

$$
V_{0}(y)= \begin{cases}-\frac{1}{2} \Lambda Q(y) & \text { for } m=2, \\ -Q^{2}(y) & \text { for } m=3, \\ \frac{1}{3}\left[Q^{\prime}(y) \int_{0}^{y} Q^{2}-2 Q^{3}(y)\right] & \text { for } m=4 .\end{cases}
$$




\section{Construction of a soliton-like solution}

Following the plan on page 583, we deal first with large negative times, by finding a pure soliton-like solution of (1-15) that agrees as $t \rightarrow-\infty$, to exponential order in time, with $Q(\cdot-(1-\lambda) t)$, where $Q$ is a soliton for the gKdV equation. Specifically:

Theorem 3.1 (Existence and uniqueness of a pure soliton-like solution). Suppose $0 \leq \lambda<1$. There exists $\varepsilon_{0}>0$ small enough such that the following holds for any $0<\varepsilon<\varepsilon_{0}$.

(1) Existence. There exists a solution $u \in C\left(\mathbb{R}, H^{1}(\mathbb{R})\right)$ of (1-15) such that

$$
\lim _{t \rightarrow-\infty}\|u(t)-Q(\cdot-(1-\lambda) t)\|_{H^{1}(\mathbb{R})}=0,
$$

and energy $E_{a}[u](t)=\left(\lambda-\lambda_{0}\right) M[Q]$. Moreover, there exist constants $K, \gamma>0$ such that for all time $t \leq-\frac{1}{2} T_{\varepsilon}$ and $s \geq 1$,

$$
\|u(t)-Q(\cdot-(1-\lambda) t)\|_{H^{s}(\mathbb{R})} \leq K \varepsilon^{-1} e^{\varepsilon \gamma t} .
$$

In particular,

$$
\left\|u\left(-T_{\varepsilon}\right)-Q\left(\cdot+(1-\lambda) T_{\varepsilon}\right)\right\|_{H^{1}(\mathbb{R})} \leq K \varepsilon^{-1} e^{-\gamma \varepsilon^{-\frac{1}{100}}} \leq K \varepsilon^{10} .
$$

(2) Uniqueness. This solution is unique if $m=3$, or if $m=2,4$ and $0<\lambda<1$.

The proof is outlined in Section A.1, and is closely modeled on [Martel 2005], where the existence of a unique $\mathrm{N}$-soliton solution for gKdV equations was established. Other proofs exist, but Martel's method has the advantage of giving an explicit uniform bound in time, (3-2). (This bound is a consequence of compactness properties.) Basically, the result follows from the fact that inside the region $x \leq-\frac{1}{2} T_{\varepsilon}$ the potential $a_{\varepsilon}$ equals 1 to exponential order (see (1-13)). In other words, the aKdV equation (1-15) behaves asymptotically as a $\mathrm{gKdV}$ equation, for which soliton solutions exist globally.

Remarks. (a) The energy identity in part (1) of the theorem follows from (3-1), the identities in Section A.6, and the energy conservation law in Proposition 2.1.

(b) The uniqueness of $u(t)$ in the general case is an interesting open question.

(c) For the solution $u(t)$ given by Theorem 3.1, it follows easily from the negativity of the energy $E_{a}$ and the Gagliardo-Nirenberg inequality (1-8)

$$
\frac{1}{K}\|u(t)\|_{H^{1}(\mathbb{R})} \leq\|u(t)\|_{L^{2}(\mathbb{R})} \leq K\|u(t)\|_{H^{1}(\mathbb{R})} \quad \text { for all } t \in \mathbb{R},
$$

for some constant $K>0$. Moreover, if $m=3$ or $m=2,4$ and $\lambda>0$, we have

$$
\sup _{t \in \mathbb{R}}\|u(t)\|_{H^{1}(\mathbb{R})} \leq K\left\|u\left(-\frac{1}{2} T_{\varepsilon}\right)\right\|_{H^{1}(\mathbb{R})} .
$$

This last estimate shows that, to understand the limiting behavior at large times of $u(t)$, it is enough to consider the $L^{2}$-norm. 


\section{Description of the soliton-potential interaction}

Once we have proven the existence (and uniqueness) of a pure soliton-like solution for early times, the next step in the study of the soliton-potential interaction. This nonlinear interaction regime is essentially limited to the region $\left[-T_{\varepsilon}, T_{\varepsilon}\right]$, since $a_{\varepsilon}\left(-T_{\varepsilon}\right) \sim 1$ and $a_{\varepsilon}\left(T_{\varepsilon}\right) \sim 2$, by (1-12) and (1-13).

Here we have a stability result saying that the soliton survives the interaction, that the perturbation induced by the potential $a_{\varepsilon}$ is significant, of order one, and that it affects the scaling and shift parameters (the scaling being predicted by conservation of energy). The soliton exits the interaction region as a first-order solution of the aKdV equation (1-15) with $a_{\varepsilon} \equiv 2$, plus a dispersive term of order $\varepsilon^{1 / 2}$ in $H^{1}(\mathbb{R})$.

Recall that we defined $\lambda_{0}:=\frac{5-m}{m+3}$ in Theorem 1.1. This parameter plays a crucial role in determining the asymptotic behavior.

Theorem 4.1 (Dynamics of the soliton in the interaction region). Suppose $0 \leq \lambda \leq \lambda_{0}$. There exist constants $\varepsilon_{0}>0$ and $c_{\infty}(\lambda)>1$ such that the following holds for any $0<\varepsilon<\varepsilon_{0}$. Let $u=u(t)$ be $a$ globally defined $H^{1}$ solution of (1-15) such that

$$
\left\|u\left(-T_{\varepsilon}\right)-Q\left(\cdot+(1-\lambda) T_{\varepsilon}\right)\right\|_{H^{1}(\mathbb{R})} \leq K \varepsilon^{1 / 2} .
$$

Then there exist $K_{0}=K_{0}(K)>0$ and $\rho\left(T_{\varepsilon}\right), \rho_{1}\left(T_{\varepsilon}\right) \in \mathbb{R}$ such that

$$
\left\|u\left(T_{\varepsilon}+\rho_{1}\left(T_{\varepsilon}\right)\right)-2^{-1 /(m-1)} Q_{c_{\infty}}\left(\cdot-\rho\left(T_{\varepsilon}\right)\right)\right\|_{H^{1}(\mathbb{R})} \leq K_{0} \varepsilon^{1 / 2} .
$$

In addition, $c_{\infty}(\lambda=0)=2^{p}$, with $p=\frac{4}{m+3}$, and $c_{\infty}\left(\lambda=\lambda_{0}\right)=1$. Finally, for $\varepsilon_{0}$ sufficiently small, we have

$$
\left|\rho_{1}\left(T_{\varepsilon}\right)\right| \leq \frac{T_{\varepsilon}}{100} \quad \text { and } \quad(1-\lambda) T_{\varepsilon} \leq \rho\left(T_{\varepsilon}\right) \leq\left(2 c_{\infty}(\lambda)-\lambda-1\right) T_{\varepsilon}
$$

Remarks. (a) Though Theorem 3.1 ensures exponential decay for the error term at time $t=-T_{\varepsilon}-$ see (3-3) and (4-1) - we are unable to get a better estimate on the solution at time $t=T_{\varepsilon}$. This is due to the emergence of dispersive terms of order $\varepsilon^{1 / 2}$, hard to describe using soliton-based functions. This new phenomenon is similar to a recent description obtained by Martel and Merle [2011; 2010] for the collision of two solitons of similar sizes for the BBM and $\mathrm{KdV}$ equations.

(b) We do not know whether Theorem 4.1 is still valid in the range $\lambda>\lambda_{0}$. Computations suggest that in this regime the soliton might be reflected after the interaction. We hope to consider this regime in a forthcoming publication. (See $[\mathrm{Muñoz} \geq 2011 \mathrm{a}]$.)

As mentioned in the Introduction, to prove this theorem we first construct an approximate solution of (1-15) that describes the first-order interaction between the soliton and the potential on the interval of time $\left[-T_{\varepsilon}, T_{\varepsilon}\right]$. This requires several steps and will occupy us for the rest of this section, culminating in Proposition 4.7. Then, in Section 5, we will prove that the actual solution describing the interaction of the soliton and the potential $a_{\varepsilon}$ is sufficiently close to our approximate solution.

Our first step is the introduction of suitable notation. 
Decomposition of the approximate solution. We look for $\tilde{u}(t, x)$, the approximate solution for (1-1), as a suitable modulation of the soliton $Q(x-(1-\lambda) t)$, which solves the $\mathrm{KdV}$ equation

$$
u_{t}+\left(u_{x x}-\lambda u+u^{m}\right)_{x}=0
$$

Let $c=c(\varepsilon t) \geq 1$ be a bounded function to be chosen later and set

$$
y:=x-\rho(t), \quad R(t, x):=\frac{Q_{c(\varepsilon t)}(y)}{\tilde{a}(\varepsilon \rho(t))},
$$

where

$$
\tilde{a}(s):=a^{\frac{1}{m-1}}(s), \quad \rho(t):=-(1-\lambda) T_{\varepsilon}+\int_{-T_{\varepsilon}}^{t}(c(\varepsilon s)-\lambda) d s .
$$

The parameter $\tilde{a}$ is intended to describe the shape variation of the soliton along the interaction.

The form of $\tilde{u}(t, x)$ will be the sum of the soliton plus a correction term:

$$
\begin{aligned}
\tilde{u}(t, x) & :=R(t, x)+w(t, x), \\
w(t, x) & :=\varepsilon A_{c}(\varepsilon t ; y),
\end{aligned}
$$

where $A_{c}:=A_{c(\varepsilon t)}(\varepsilon t ; y)=c^{\frac{1}{m-1}} A(\varepsilon t ; \sqrt{c} y)$ and $A$ is a function to be determined.

We want to estimate the error produced by inserting $\tilde{u}$ as defined in (4-8) into (1-1). For this, let

$$
S[\tilde{u}](t, x):=\tilde{u}_{t}+\left(\tilde{u}_{x x}-\lambda \tilde{u}+a_{\varepsilon} \tilde{u}^{m}\right)_{x} .
$$

Proposition 4.2 (First decomposition of $S[\tilde{u}]$ ). Let $\mathscr{L}$ be the linear operator defined in (2-9). The following nonlinear decomposition of the error term $S[\tilde{u}]$ is valid for every $t \in\left[-T_{\varepsilon}, T_{\varepsilon}\right]$ :

$$
S[\tilde{u}](t, x)=\varepsilon\left(F_{1}-\left(\mathscr{L} A_{c}\right)_{y}\right)(\varepsilon t ; y)+\varepsilon^{2}\left(\left(A_{c}\right)_{t}+c^{\prime}(\varepsilon t) \Lambda A_{c}\right)(\varepsilon t ; y)+\varepsilon^{2} \mathscr{E}(t, x),
$$

where $\Lambda A_{c}(y):=\frac{1}{c}\left(\frac{1}{m-1} A_{c}(y)+\frac{1}{2} y\left(A_{c}\right)_{y}(y)\right)$ (compare Lemma 2.3),

$$
F_{1}(\varepsilon t ; y):=\frac{c^{\prime}(\varepsilon t)}{\tilde{a}(\varepsilon \rho(t))} \Lambda Q_{c}(y)+\frac{a^{\prime}(\varepsilon \rho(t))}{\tilde{a}^{m}(\varepsilon \rho(t))}\left(-\frac{1}{m-1}(c(\varepsilon t)-\lambda) Q_{c}(y)+\left(y Q_{c}^{m}(y)\right)_{y}\right)
$$

and $\mathscr{E}(t, x)$ is a bounded function in $\left[-T_{\varepsilon}, T_{\varepsilon}\right] \times \mathbb{R}$.

We prove this result in Section A.2.

Next, if we want to improve the approximation $\tilde{u}$, the unknown function $A_{c}$ must be such that

$$
\left(\mathscr{L} A_{c}\right)_{y}(\varepsilon t ; y)=F_{1}(\varepsilon t ; y) \quad \text { for all } y \in \mathbb{R} .
$$

Then the error term will be reduced to the second-order quantity

$$
S[\tilde{u}]=\varepsilon^{2}\left[\left(A_{c}\right)_{t}+c^{\prime}(\varepsilon t) \Lambda A_{c}\right](\varepsilon t ; y)+\varepsilon^{2} \mathscr{E}(t, x) .
$$

We prove the solvability of $(\Omega)$, which is of independent interest, in the next few pages, concluding with Lemma 4.5. However, we will see that it is not always possible to find a solution of finite mass. In fact, we will look for solutions such that time and space variables are separated:

$$
A_{c}(t, y)=b(\varepsilon t) \varphi_{c}(y)+d(\varepsilon t)+h(\varepsilon t) \hat{A}_{c}(y) ;
$$


where $b(s), d(s)$ and $h(s)$ are exponentially decreasing in $s, \varphi_{c}$ is the bounded function defined in (2-11) and $\hat{A}_{c} \in Y$ (recall that $\lim _{ \pm \infty} \varphi_{c}= \pm \sqrt{c}$, and that $c \geq 1$ ).

This choice is motivated by the fact that a function $A_{c}$ as in (4-11) satisfies this Important Property:

Property IP. Any spatial derivative of $A_{c}(\varepsilon t, \cdot)$ is a localized $y$-function, and there exist $K, \gamma>0$ such that $\left\|A_{c}(\varepsilon t, \cdot)\right\|_{L^{\infty}(\mathbb{R})} \leq K e^{-\gamma \varepsilon|t|}$ for all $t \in \mathbb{R}$.

Solution of a time-independent model problem. As a stepping stone to the solution of $(\Omega)$, we address the following existence problem. Given a bounded, even function $F=F(y)$, we seek a bounded solution $A=A(y)$ to the model problem

$$
\left(\mathscr{L}_{0} A\right)^{\prime}=F,
$$

where $\mathscr{L}_{0}:=-\partial_{y y}^{2}+1-m Q^{m-1}(y)$ as in (2-9). In the spirit of [Martel and Merle 2007, Proposition 2.1] and [Muñoz 2010, Proposition 3.2], we have:

Lemma 4.3 (Existence theory for (4-12)). Let $F \in \mathcal{Y}$ be even and satisfy the orthogonality condition

$$
\int_{\mathbb{R}} F Q=0
$$

Let $\beta=\frac{1}{2} \int_{\mathbb{R}} F$. For any $\delta \in \mathbb{R}$, the problem (4-12) has a bounded solution $A$ of the form

$$
A(y)=\beta \varphi(y)+\delta+A_{1}(y), \quad \text { with } A_{1}(y) \in \mathscr{Y} .
$$

This solution is unique in $L^{2}(\mathbb{R})$ up to the addition of a constant times $Q^{\prime}$.

Proof. Write $A:=\beta \varphi+\delta\left(1+V_{0}\right)+A_{1}$, where $\beta, \delta \in \mathbb{R}$ and $A_{1} \in \mathcal{Y}$ are to be determined. Inserting this decomposition in (4-12), we have $\left(\mathscr{L}_{0} A_{1}\right)^{\prime}=F-\beta\left(\mathscr{L}_{0} \varphi\right)^{\prime}$, namely

$$
\mathscr{L}_{0} A_{1}=H-\beta \mathscr{L}_{0} \varphi+\gamma, \quad H(y):=\int_{-\infty}^{y} F(s) d s,
$$

and where $\gamma:=\mathscr{L}_{0} A_{1}(0)-\int_{-\infty}^{0} H(s) d s$. Without loss of generality we can suppose the constant term $\gamma$ equals $-\beta$, because from Lemma 2.4 we have $\mathscr{L}_{0}\left(1+V_{0}\right)=1$, so any constant term can be associated to the free parameter $\delta$.

Now, from Lemma 2.3 the problem (4-12) is solvable if and only if

$$
\int_{\mathbb{R}}\left(H-\beta\left(\mathscr{L}_{0} \varphi+1\right)\right) Q^{\prime}=\int_{\mathbb{R}} H Q^{\prime}=\int_{\mathbb{R}} F Q=0,
$$

which is (4-13) (recall that $\mathscr{L}_{0} Q^{\prime}=0$ ). Thus there exists a solution $A_{1}$ of (4-15) satisfying $\int_{\mathbb{R}} A_{1} Q^{\prime}=0$. Moreover, since

$$
\lim _{y \rightarrow-\infty}\left(H-\beta\left(\mathscr{L}_{0} \varphi+1\right)\right)(y)=0 \quad \text { and } \quad \lim _{y \rightarrow+\infty}\left(H-\beta\left(\mathscr{L}_{0} \varphi+1\right)\right)(y)=\int_{\mathbb{R}} F-2 \beta,
$$

we get $A_{1} \in \mathcal{Y}$ provided $\beta=\frac{1}{2} \int_{\mathbb{R}} F$, by Lemma 2.3. This finishes the proof. 
Existence of dynamical parameters. Now we show the existence of a dynamical system involving the evolution of first-order scaling and translation parameters on the main interaction region. This system is related to the orthogonality condition $\int_{\mathbb{R}} F_{1} Q_{c}=0$; see proof of Lemma 4.5 below.

Lemma 4.4 (Existence of dynamical parameters). Let $m=2,3,4$ and let $\lambda_{0}, p, a(\cdot)$ be as in Theorem 4.1 and (1-13). The system of of differential equations

$$
\begin{cases}c^{\prime}(\varepsilon t)=p c(\varepsilon t)\left(c(\varepsilon t)-\frac{\lambda}{\lambda_{0}}\right) \frac{a^{\prime}}{a}(\varepsilon \rho(t)), & c\left(-\varepsilon T_{\varepsilon}\right)=1, \\ \rho^{\prime}(t)=c(\varepsilon t)-\lambda, & \rho\left(-T_{\varepsilon}\right)=-(1-\lambda) T_{\varepsilon}\end{cases}
$$

has a unique solution $(\rho, c)$ with $c$ bounded, positive, monotone, defined for all $t \geq-T_{\varepsilon}$, and having the same regularity as $a(\varepsilon \cdot)$. In addition:

(1) If $\lambda=\lambda_{0}$, one has $c \equiv 1$.

(2) If $0 \leq \lambda<\lambda_{0}$ then for all $t \geq-T_{\varepsilon}$ one has $c(\varepsilon t)>1$ and $\lim _{t \rightarrow+\infty} c(\varepsilon t)=c_{\infty}+O\left(\varepsilon^{10}\right)$, where $c_{\infty}=c_{\infty}(\lambda)>1$ is the unique solution of the algebraic equation

$$
c_{\infty}^{\lambda_{0}}\left(c_{\infty}-\frac{\lambda}{\lambda_{0}}\right)^{1-\lambda_{0}}=2^{p}\left(1-\frac{\lambda}{\lambda_{0}}\right)^{1-\lambda_{0}}, \quad c_{\infty}>1 .
$$

Moreover, $\lambda \in\left[0, \lambda_{0}\right] \mapsto c_{\infty}(\lambda) \geq 1$ is a smooth decreasing function and $c_{\infty}(\lambda=0)=2^{p}$.

In the case $\lambda=0$, there exists a simple implicit expression for $c(\varepsilon t)$ :

$$
\rho^{\prime}(t)=c(\varepsilon t)=\frac{a^{p}(\varepsilon \rho(t))}{a^{p}\left(-\varepsilon T_{\varepsilon}\right)} .
$$

Using the strict monotonicity of $a$, from this identity we can find explicitly $c(\varepsilon t)$.

Remark. The critical value $\lambda_{0}$ can be seen as the exact value of $\lambda$ such that the solution $u(t)$ constructed in Theorem 3.1 has zero energy. Indeed, Theorem 3.1 gives $E_{a}[u]=\left(\lambda-\lambda_{0}\right) M[Q]$. Hence $E_{a}[u]$ is zero, positive or negative depending on whether $\lambda=\lambda_{0}, \lambda>\lambda_{0}$, or $\lambda<\lambda_{0}$. Because of this the study of the soliton dynamics for $\lambda>\lambda_{0}$ is an open question.

Proof of Lemma 4.4. The local existence of a solution $(c, \rho)$ of (4-16) is a direct consequence of the Cauchy-Lipschitz-Picard theorem.

Now we use (4-16) to prove a priori estimates on the solution $c$. Note that

$$
\frac{c(\varepsilon t)-\lambda}{c(\varepsilon t)\left(c(\varepsilon t)-\frac{\lambda}{\lambda_{0}}\right)} c^{\prime}(\varepsilon t)=p(c(\varepsilon t)-\lambda) \frac{a^{\prime}}{a}(\varepsilon \rho)=p \rho^{\prime}(t) \frac{a^{\prime}}{a}(\varepsilon \rho) \text {. }
$$

In particular,

$$
\left(1-\lambda_{0}\right) \partial_{t} \log \left(c(\varepsilon t)-\frac{\lambda}{\lambda_{0}}\right)+\lambda_{0} \partial_{t} \log c(\varepsilon t)=p \partial_{t} \log a(\varepsilon \rho) .
$$


Integrating on $\left[-T_{\varepsilon}, t\right]$ and using $c\left(-\varepsilon T_{\varepsilon}\right)=1$, we obtain

$$
c^{\lambda_{0}}(\varepsilon t)\left(c(\varepsilon t)-\frac{\lambda}{\lambda_{0}}\right)^{1-\lambda_{0}}=\left(1-\frac{\lambda}{\lambda_{0}}\right)^{1-\lambda_{0}} \frac{a^{p}(\varepsilon \rho(t))}{a^{p}\left(-\varepsilon(1-\lambda) T_{\varepsilon}\right)} .
$$

Since $1 \leq a \leq 2$, the function $c$ is bounded and $\rho$ is bounded on compact sets; this yields global existence. One proves in particular that $c^{\prime}>0$ and

$$
c^{\lambda_{0}}(\varepsilon t)<a^{p}(\varepsilon \rho), \quad \text { and thus } \quad 1 \leq c(\varepsilon t) \leq 2^{\frac{4}{5-m}} .
$$

The limit $\lim _{t \rightarrow+\infty} c(\varepsilon t)$ exists and is of the form $c_{\infty}+O\left(\varepsilon^{10}\right)$, where $c_{\infty}$ is a solution of (4-17), after passing to the limit in (4-18). To prove the uniqueness of the solution of (4-17), consider for $\mu \geq 1$ the smooth function

$$
g(\mu ; \lambda):=\mu^{\lambda_{0}}\left(\mu-\frac{\lambda}{\lambda_{0}}\right)^{1-\lambda_{0}}-2^{p}\left(1-\frac{\lambda}{\lambda_{0}}\right)^{1-\lambda_{0}} .
$$

Note that in the case $\lambda<\lambda_{0}$ we have $g(1 ; \lambda)<0$ and

$$
\partial_{\mu} g(\mu ; \lambda)=\mu^{\lambda_{0}-1}\left(\mu-\frac{\lambda}{\lambda_{0}}\right)^{-\lambda_{0}}(\mu-\lambda) \geq\left(1-\frac{\lambda}{\lambda_{0}}\right)^{-\lambda_{0}}>0 .
$$

This implies that there exists a unique $c_{\infty}(\lambda)>1$ such that $g\left(c_{\infty}(\lambda) ; \lambda\right)=0$. This proves uniqueness. The smoothness of the application $\lambda \in\left[0, \lambda_{0}\right] \mapsto c_{\infty}(\lambda)$ is an easy consequence of the implicit function theorem.

Finally we prove that $\lambda \mapsto c_{\infty}(\lambda)$ is a decreasing map. To do this, we differentiate (4-17), obtaining

$$
\begin{aligned}
\frac{c_{\infty}(\lambda)^{\lambda_{0}-1}\left(c_{\infty}(\lambda)-\lambda\right)}{\left(c_{\infty}(\lambda)-\frac{\lambda}{\lambda_{0}}\right)^{\lambda_{0}}} c_{\infty}^{\prime}(\lambda) & =\left(\frac{1}{\lambda_{0}}-1\right)\left(\frac{c_{\infty}^{\lambda_{0}}(\lambda)}{\left(c_{\infty}(\lambda)-\frac{\lambda}{\lambda_{0}}\right)^{\lambda_{0}}}-\frac{2^{p}}{\left(1-\frac{\lambda}{\lambda_{0}}\right)^{\lambda_{0}}}\right) \\
& \leq\left(\frac{1}{\lambda_{0}}-1\right)\left(1-\frac{\lambda}{\lambda_{0}}\right)^{-\lambda_{0}}\left(1-2^{p}\right)<0 .
\end{aligned}
$$

We can now state the promised result on the solvability of $(\Omega)$ :

Lemma 4.5. Suppose $0 \leq \lambda \leq \lambda_{0}$ and $c(\varepsilon t)$ is given by (4-16). There exists a solution $A_{c}=A_{c}(\varepsilon t ; y)$ of

$$
\left(\mathscr{L} A_{c}\right)^{\prime}(\varepsilon t ; y)=F_{1}(\varepsilon t ; y)
$$

satisfying Property IP and the following conditions:

(1) For every $t \in\left[-T_{\varepsilon}, T_{\varepsilon}\right]$,

$$
\begin{cases}A_{c}(\varepsilon t ; \cdot) \in L^{\infty}(\mathbb{R}), & A_{c}(\varepsilon t ; y)=b(\varepsilon t)\left(\varphi_{c}(y)-c^{1 / 2}\right)+h(\varepsilon t) \hat{A}_{c}(y), \\ \hat{A}_{c} \in Y, & |b(\varepsilon t)|+|h(\varepsilon t)| \leq K e^{-\gamma \varepsilon|t|} .\end{cases}
$$

(2) $\lim _{y \rightarrow+\infty} A_{c}(y)=0$.

Remark. The function $A_{c}$ models, to first order in $\varepsilon$, the shelf-like tail behind the soliton, a dispersive effect of the soliton-potential interaction. 
Proof. Step 1: Reduction to a time-independent problem. We suppose $c$ given as in Lemma 4.4. Note that $F_{1}$ in $\overline{(4-10)}$ can be written as

$$
F_{1}(\varepsilon t ; y)=\frac{a^{\prime}}{\tilde{a}^{m}}\left[p c\left(c-\frac{\lambda}{\lambda_{0}}\right) \Lambda Q_{c}-\frac{1}{m-1}(c-\lambda) Q_{c}+\left(y Q_{c}^{m}\right)^{\prime}\right](y) .
$$

Consider the functions

$$
\tilde{F}_{1}(y):=p \Lambda Q-\frac{1}{m-1} Q+\left(y Q^{m}\right)^{\prime} \quad \text { and } \quad \hat{F}_{1}(y):=\frac{1}{m-1} Q-\frac{p}{\lambda_{0}} \Lambda Q=\frac{1}{m-1} Q-\frac{4}{5-m} \Lambda Q .
$$

We claim that if $c(\varepsilon t)$ satisfies (4-16) then every term in $F_{1}$ has the correct scaling. More precisely:

Claim 4.6. Suppose $\tilde{A}(y), \hat{A}(y)$ solve the stationary problems

$$
\left(\mathscr{L}_{0} \tilde{A}\right)^{\prime}=\tilde{F}_{1}, \quad\left(\mathscr{L}_{0} \hat{A}\right)^{\prime}=\hat{F}_{1} .
$$

Then, for all $t \in \mathbb{R}$,

$$
A_{c}(\varepsilon t ; y):=\frac{a^{\prime}(\varepsilon \rho)}{\tilde{a}^{m}(\varepsilon \rho)} c^{\frac{1}{m-1}-\frac{1}{2}}(\varepsilon t)\left(\tilde{A}+\lambda c^{-1}(\varepsilon t) \hat{A}\right)\left(c^{1 / 2}(\varepsilon t) y\right)
$$

is a solution of (4-20).

Indeed, we have

$$
\begin{aligned}
& \left(\mathscr{L} A_{c}\right)^{\prime} \\
& =\frac{a^{\prime}(\varepsilon \rho)}{\tilde{a}^{m}(\varepsilon \rho)} c^{\frac{1}{m-1}+1}\left(-\tilde{A}^{\prime \prime}+\tilde{A}-m Q^{m-1} \tilde{A}\right)^{\prime}\left(c^{1 / 2} y\right)+\lambda \frac{a^{\prime}(\varepsilon \rho)}{\tilde{a}^{m}(\varepsilon \rho)} c^{\frac{1}{m-1}}\left(-\hat{A}^{\prime \prime}+\hat{A}-m Q^{m-1} \hat{A}\right)^{\prime}\left(c^{1 / 2} y\right) \\
& =\frac{a^{\prime}(\varepsilon \rho)}{\tilde{a}^{m}(\varepsilon \rho)} c^{\frac{1}{m-1}+1} \tilde{F}_{1}\left(c^{1 / 2} y\right)+\lambda \frac{a^{\prime}(\varepsilon \rho)}{\tilde{a}^{m}(\varepsilon \rho)} c^{\frac{1}{m-1}} \hat{F}_{1}\left(c^{1 / 2} y\right) \\
& =\frac{a^{\prime}(\varepsilon \rho)}{\tilde{a}^{m}(\varepsilon \rho)}\left(p c^{2} \Lambda Q_{c}-\frac{1}{m-1} c Q_{c}+\left(y Q_{c}^{m}\right)^{\prime}\right)+\lambda \frac{a^{\prime}(\varepsilon \rho)}{\tilde{a}^{m}(\varepsilon \rho)}\left(\frac{1}{m-1} Q_{c}-\frac{p}{\lambda_{0}} c \Lambda Q_{c}\right)=F_{1}(\varepsilon t ; y)
\end{aligned}
$$

This proves the claim, which in turn reduces the problem to the time-independent case.

Step 2: There exists solutions $\tilde{A}, \hat{A}$ of (4-22) satisfying (4-14). By Lemma 4.3, this follows once we verify the orthogonality conditions

$$
\int_{\mathbb{R}} \tilde{F}_{1} Q=\int_{\mathbb{R}} \hat{F}_{1} Q=0
$$

To this end, we use the identities in Section A.6:

$$
\begin{aligned}
\int_{\mathbb{R}} \tilde{F}_{1} Q & =p \int_{\mathbb{R}} \Lambda Q Q-\frac{1}{m-1} \int_{\mathbb{R}} Q^{2}+\int_{\mathbb{R}} Q\left(y Q^{m}\right)_{y} \\
& =p \int_{\mathbb{R}} \Lambda Q Q-\frac{1}{m-1} \int_{\mathbb{R}} Q^{2}+\frac{1}{m+1} \int_{\mathbb{R}} Q^{m+1}=\frac{5-m}{4(m-1)}\left(p-\frac{4}{m+3}\right) \int_{\mathbb{R}} Q^{2}=0 .
\end{aligned}
$$

Similarly,

$$
\int_{\mathbb{R}} \hat{F}_{1} Q=-\frac{4}{5-m} \int_{\mathbb{R}} \Lambda Q Q+\frac{1}{m-1} \int_{\mathbb{R}} Q^{2}=-\frac{4}{5-m} \times \frac{5-m}{4(m-1)} \int_{\mathbb{R}} Q^{2}+\frac{1}{m-1} \int_{\mathbb{R}} Q^{2}=0 .
$$


Thus, by virtue of Lemma 4.3, there exist solutions $\tilde{A}, \hat{A}$ of (4-22) of the form

$$
\begin{cases}\tilde{A}(y)=\tilde{\beta} \varphi(y)+\tilde{\delta}+\tilde{A}_{1}(y), \quad \tilde{A}_{1} \in \mathscr{Y}, \\ \hat{A}(y)=\hat{\beta} \varphi(y)+\hat{\delta}+\hat{A}_{1}(y), \quad \hat{A}_{1} \in \mathscr{Y},\end{cases}
$$

where $\tilde{\beta}, \hat{\beta}, \tilde{\delta}, \hat{\delta} \in \mathbb{R}$. Moreover, $\tilde{\beta}, \hat{\beta}$ are given by

$$
\tilde{\beta}:=\frac{1}{2} \int_{\mathbb{R}} \tilde{F}_{1}=\frac{1}{2} \int_{\mathbb{R}}\left(p \Lambda Q-\frac{1}{m-1} Q\right)=\frac{1}{2}\left(p\left(\frac{1}{m-1}-\frac{1}{2}\right)-\frac{1}{m-1}\right) \int_{\mathbb{R}} Q=-\frac{3}{2(m+3)} \int_{\mathbb{R}} Q<0,
$$

for each $m=2,3,4$. On the other hand,

$\hat{\beta}:=\frac{1}{2} \int_{\mathbb{R}} \hat{F}_{1}=\frac{1}{2} \int_{R}\left(\frac{1}{m-1} Q-\frac{4}{5-m} \Lambda Q\right)=\frac{1}{2}\left(\frac{1}{m-1}-\frac{4}{5-m} \times \frac{3-m}{2(m-1)}\right) \int_{\mathbb{R}} Q=\frac{1}{2(5-m)} \int_{\mathbb{R}} Q>0$, for each $m=2,3,4$.

Step 3: Conclusion. Finally, to get $\lim _{y \rightarrow+\infty} \tilde{A}(y)=\lim _{y \rightarrow+\infty} \hat{A}(y)=0$ we choose $\tilde{\delta}=-\tilde{\beta}$ and $\widehat{\delta}=-\hat{\beta}$. This proves the last part of the lemma. With this choice we have

$$
\tilde{A}(y)=\tilde{\beta}(\varphi(y)-1)+\tilde{A}_{1}(y), \quad \hat{A}(y)=\hat{\beta}(\varphi(y)-1)+\hat{A}_{1}(y), \quad \tilde{A}_{1}, \hat{A}_{1} \in \mathscr{Y} .
$$

Using Claim 4.6, an actual solution $A_{c}(\varepsilon t ; y)$ of (4-20) is obtained by considering

$$
\begin{aligned}
A_{c}(\varepsilon t ; y) & :=\frac{a^{\prime}(\varepsilon \rho)}{\tilde{a}^{m}(\varepsilon \rho)} c^{\frac{1}{m-1}}(\varepsilon t)\left[\tilde{A}+\lambda c^{-1}(\varepsilon t) \hat{A}\right]\left(c^{1 / 2} y\right) \\
& =: b(\varepsilon t)\left(\varphi_{c}(y)-c^{1 / 2}\right)+h(\varepsilon t) \hat{A}_{c}(y), \quad \hat{A}_{c} \in \mathscr{Y},
\end{aligned}
$$

where

$$
b(\varepsilon t):=\frac{a^{\prime}(\varepsilon \rho) c^{\frac{1}{m-1}-1}}{\tilde{a}^{m}(\varepsilon \rho)}\left(\tilde{\beta}+\lambda c^{-1}(\varepsilon t) \hat{\beta}\right), \quad h(\varepsilon t):=\frac{a^{\prime}(\varepsilon \rho)}{\tilde{a}^{m}(\varepsilon \rho)} .
$$

This finishes the proof of Lemma 4.5 .

Remark. We emphasize that $A_{c}$ lies in $L^{2}(\mathbb{R})$ in all cases, even if it is exponentially decreasing in time. This nonsummable solution must be modified in order to obtain a finite-mass solution.

Before continuing with the construction of the approximate solution, we need some crucial estimates on the parameter $c(\varepsilon t)$.

Remark (Bounds for $c(\varepsilon t)$ ). From the bound on $c(\varepsilon t)$ in (4-18) we conclude that, for all $t \in\left[-T_{\varepsilon}, T_{\varepsilon}\right]$,

$$
1 \leq c(\varepsilon t) \leq 2 \frac{4}{5-m}
$$

Correction to the solution of problem $(\Omega)$. Consider the cutoff function $\eta \in C^{\infty}(\mathbb{R})$ satisfying the following properties:

$$
\begin{cases}0 \leq \eta(s) \leq 1, \quad 0 \leq \eta^{\prime}(s) \leq 1 & \text { for any } s \in \mathbb{R} \\ \eta(s) \equiv 0 & \text { for } s \leq-1 \\ \eta(s) \equiv 1 & \text { for } s \geq 1\end{cases}
$$

Define

$$
\eta_{\varepsilon}(y):=\eta(\varepsilon y+2)
$$


and for the solution $A_{c}=A_{c}(\varepsilon t ; y)$ of (4-20) constructed in Lemma 4.5, set

$$
A_{\#}(\varepsilon t ; y):=\eta_{\varepsilon}(y) A_{c}(\varepsilon t ; y) .
$$

Now redefine

$$
\tilde{u}:=R+w=R+\varepsilon A_{\#} .
$$

where $R$ is the modulated soliton from (4-5).

The following proposition, which deals with the error associated to this cut-off function and the new approximate solution $\tilde{u}$, is the principal result of this section.

Proposition 4.7 (Construction of an approximate solution for (1-15)). There exist constants $\varepsilon_{0}, K>0$ such that for all $0<\varepsilon<\varepsilon_{0}$ the following holds.

(1) For the localized function $A_{\#}$ of (4-25), we have:

(a) New behavior. For all $t \in\left[-T_{\varepsilon}, T_{\varepsilon}\right]$,

$$
\begin{cases}A_{\#}(\varepsilon t, y)=0 & \text { for all } y \leq-\frac{3}{\varepsilon}, \\ A_{\#}(\varepsilon t, y)=A_{c}(\varepsilon t, y) & \text { for all } y \geq-\frac{1}{\varepsilon} .\end{cases}
$$

(b) Integrable solution. For all $t \in\left[-T_{\varepsilon}, T_{\varepsilon}\right], A_{\#}(\varepsilon t, \cdot) \in H^{1}(\mathbb{R})$ with

$$
\left\|\varepsilon A_{\#}(\varepsilon t, \cdot)\right\|_{H^{1}(\mathbb{R})} \leq K \varepsilon^{\frac{1}{2}} e^{-\gamma \varepsilon|t|} .
$$

(2) The error associated to the new function $\tilde{u}$ satisfies

$$
\|S[\tilde{u}](t)\|_{H^{2}(\mathbb{R})} \leq K \varepsilon^{\frac{3}{2}} e^{-\gamma \varepsilon|t|},
$$

and the following integral estimate holds:

$$
\int_{\mathbb{R}}\|S[\tilde{u}](t)\|_{H^{2}(\mathbb{R})} d t \leq K \varepsilon^{1 / 2} .
$$

Proof. The proof of (4-27) is direct from the definition. To prove (4-28) it is enough to recall that

$$
\left\|\eta_{c}^{\prime}\right\|_{L^{2}(\mathbb{R})} \leq K \varepsilon^{-1 / 2} .
$$

For the proof of (4-29), see Section A.3.

Recomposition of the solution. We now present some important estimates concerning our approximate solution, showing that $\tilde{u}$ at time $\pm T_{\varepsilon}$ behaves as a modulated soliton with the scaling given by rough computations at infinity. We start out with some model $H^{1}$-estimates.

Lemma 4.8 (First estimates on $\tilde{u}$ ).

(1) Decay away from zero. If $f=f(y) \in \mathcal{Y}$, there exist constants $K, \gamma>0$ such that, for all $t \in\left[-T_{\varepsilon}, T_{\varepsilon}\right]$,

$$
\left\|a^{\prime}(\varepsilon x) f(y)\right\|_{H^{1}(\mathbb{R})} \leq K e^{-\gamma \varepsilon|t|}
$$


(2) Almost-soliton solution. The following estimates hold for all $t \in\left[-T_{\varepsilon}, T_{\varepsilon}\right]$.

$$
\begin{gathered}
\left\|\tilde{u}_{t}+(c-\lambda) \tilde{u}_{x}\right\|_{H^{1}(\mathbb{R})} \leq K \varepsilon e^{-\gamma \varepsilon|t|}, \quad\left\|\tilde{u}_{t}+(c-\lambda) \tilde{u}_{x}\right\|_{L^{\infty}(\mathbb{R})} \leq K \varepsilon e^{-\gamma \varepsilon|t|} . \\
\tilde{u}_{x x}-\lambda \tilde{u}+a_{\varepsilon} \tilde{u}^{m}=(c-\lambda) \tilde{u}+O_{L^{2}(\mathbb{R})}\left(\varepsilon e^{-\gamma \varepsilon|t|}\right) . \\
\left\|\left(\tilde{u}_{x x}-c \tilde{u}+a_{\varepsilon} \tilde{u}^{m}\right)_{x}\right\|_{H^{1}(\mathbb{R})} \leq K \varepsilon e^{-\gamma \varepsilon|t|}+K \varepsilon^{2} .
\end{gathered}
$$

Proof. The proof of (4-30) is a direct consequence of (1-13) and the fact that $\rho^{\prime}(t)=c(\varepsilon t)-\lambda \geq 1-\lambda$, for all $t \in \mathbb{R}$.

Now let us prove (4-31). From (4-26) we obtain $\tilde{u}_{t}+(c-\lambda) \tilde{u}_{x}=\varepsilon \frac{c^{\prime}}{\tilde{a}} \Lambda Q_{c}-\varepsilon \frac{\tilde{a}^{\prime}}{\tilde{a}^{2}}(c-\lambda) Q_{c}+\varepsilon\left(\left(A_{\#}\right)_{t}+c\left(A_{\#}\right)_{x}\right)=\varepsilon\left(\left(A_{\#}\right)_{t}+c\left(A_{\#}\right)_{x}\right)+O_{H^{1}(\mathbb{R})}\left(\varepsilon e^{-\gamma \varepsilon|t|}\right)$. Now, from (A-34) in the Appendix, we know that

$$
\varepsilon\left(\left(A_{\#}\right)_{t}+c\left(A_{\#}\right)_{x}\right)=\varepsilon^{2}(c-\lambda) \eta_{c}^{\prime} A_{c}+O_{H^{1}(\mathbb{R})}\left(\varepsilon^{\frac{3}{2}} e^{-\gamma \varepsilon|t|}\right)=O_{H^{1}(\mathbb{R})}\left(\varepsilon^{\frac{3}{2}} e^{-\gamma \varepsilon|t|}\right) .
$$

This completes the proof of the $H^{1}$-estimate. The $L^{\infty}$-estimate then follows from the continuous Sobolev embedding $H^{1}(\mathbb{R}) \hookrightarrow L^{\infty}(R)$.

Concerning (4-32), note that from (4-28) we have

$$
\begin{aligned}
\tilde{u}_{x x}-\lambda \tilde{u}+a_{\varepsilon} \tilde{u}^{m} & =(c-\lambda) \tilde{u}+\varepsilon\left(\left(A_{\#}\right)_{x x}+m a_{\varepsilon} R^{m-1} A_{\#}\right)+O_{L^{2}(\mathbb{R})}\left(\varepsilon e^{-\gamma \varepsilon|t|}\right)+O\left(\varepsilon^{2}\left|A_{\#}\right|^{2}\right) \\
& =(c-\lambda) \tilde{u}+O_{L^{2}(\mathbb{R})}\left(\varepsilon e^{-\gamma \varepsilon|t|}\right) .
\end{aligned}
$$

For (4-33), note that $\left(\tilde{u}_{x x}-c \tilde{u}+a_{\varepsilon} \tilde{u}^{m}\right)_{x}=S[\tilde{u}]-\left((c-\lambda) \tilde{u}_{x}+\tilde{u}_{t}\right)$. The conclusion now follows from (4-29) and (4-31).

The next result describes the behavior of the almost solution $\tilde{u}$ at the endpoints $t=-T_{\varepsilon}, T_{\varepsilon}$.

Proposition 4.9 (Behavior at $t= \pm T_{\varepsilon}$ ). There exist constants $K, \varepsilon_{0}>0$ such that for every $0<\varepsilon<\varepsilon_{0}$ the approximate solution $\tilde{u}$ constructed in Proposition 4.7 has these properties:

(1) Closeness to $Q$ at time $t=-T_{\varepsilon}$.

$$
\left\|\tilde{u}\left(-T_{\varepsilon}\right)-Q\left(\cdot+(1-\lambda) T_{\varepsilon}\right)\right\|_{H^{1}(\mathbb{R})} \leq K \varepsilon^{10} .
$$

(2) Closeness to $2^{-1 /(m-1)} Q_{c_{\infty}}$ at time $t=T_{\varepsilon}$. Let $c_{\infty}(\lambda)>1$ be as defined in Lemma 4.4. Then

$$
\left\|\tilde{u}\left(T_{\varepsilon}\right)-2^{-1 /(m-1)} Q_{c_{\infty}}\left(\cdot-\rho\left(T_{\varepsilon}\right)\right)\right\|_{H^{1}(\mathbb{R})} \leq K \varepsilon^{10}
$$

Proof. By definition,

$$
\tilde{u}\left(-T_{\varepsilon}\right)-Q\left(\cdot-\rho\left(-T_{\varepsilon}\right)\right)=R\left(-T_{\varepsilon}\right)-Q\left(\cdot+(1-\lambda) T_{\varepsilon}\right)+w\left(-T_{\varepsilon}\right) .
$$

From Proposition 4.7 we have

$$
\left\|w\left( \pm T_{\varepsilon}\right)\right\|_{H^{1}(\mathbb{R})}=\left\|\varepsilon A_{\#}\left( \pm T_{\varepsilon}\right)\right\|_{H^{1}(\mathbb{R})} \leq K \varepsilon^{1 / 2} e^{-\gamma \varepsilon^{-\frac{1}{100}}} \leq K \varepsilon^{10}
$$


for $\varepsilon$ small enough. On the other hand, from $\rho\left(-T_{\varepsilon}\right)=-(1-\lambda) T_{\varepsilon}$ and using the monotonicity of $a$, we have

$$
1 \leq c\left(-\varepsilon T_{\varepsilon}\right) \leq a^{\frac{4}{5-m}}\left(\varepsilon \rho\left(-T_{\varepsilon}\right)\right) \leq 1+\varepsilon^{10} .
$$

In conclusion we have

$$
\left\|R\left(-T_{\varepsilon}\right)-Q\left(\cdot+(1-\lambda) T_{\varepsilon}\right)\right\|_{H^{1}(\mathbb{R})} \leq K \varepsilon^{10},
$$

as desired. The estimate (4-35) is totally analogous, and we skip the details.

To summarize this section: we have constructed and approximate solution $\tilde{u}$ describing the solitonpotential interaction in principle. In the next section we will show that the solution $u$ constructed in Theorem 3.1 actually behaves like $\tilde{u}$ inside the interaction box $\left[-T_{\varepsilon}, T_{\varepsilon}\right]$.

\section{First stability results}

Our next goal is to prove that the approximate solution $\tilde{u}$ describes the actual dynamics of interaction in the interval $\left[-T_{\varepsilon}, T_{\varepsilon}\right]$. This is the principal result of this section:

Proposition 5.1 (Exact solution close to the approximate solution $\tilde{u}$ ). Let $\kappa>\frac{1}{100}$. There exists $\varepsilon_{0}>0$ such that the following holds for any $0<\varepsilon<\varepsilon_{0}$. Suppose that

$$
\|S[\tilde{u}](t)\|_{H^{2}(\mathbb{R})} \leq K \varepsilon^{1+\kappa} e^{-\gamma \varepsilon|t|}, \quad \int_{\mathbb{R}}\|S[\tilde{u}](t)\|_{H^{2}(\mathbb{R})} d t \leq K \varepsilon^{\kappa},
$$

and

$$
\left\|u\left(-T_{\varepsilon}\right)-\tilde{u}\left(-T_{\varepsilon}\right)\right\|_{H^{1}(\mathbb{R})} \leq K \varepsilon^{\kappa},
$$

where $u=u(t)$ is an $H^{1}(\mathbb{R})$ solution of (1-15) in a vicinity of $t=-T_{\varepsilon}$. Then $u(t)$ is defined for any $t \in\left[-T_{\varepsilon}, T_{\varepsilon}\right]$ and there exist $K_{0}=K_{0}(\kappa, K)$ and a $C^{1}$-function $\rho_{1}:\left[-T_{\varepsilon}, T_{\varepsilon}\right] \rightarrow \mathbb{R}$ such that, for all $t \in\left[-T_{\varepsilon}, T_{\varepsilon}\right]$

$$
\left\|u\left(t+\rho_{1}(t)\right)-\tilde{u}(t)\right\|_{H^{1}(\mathbb{R})} \leq K_{0} \varepsilon^{\kappa}, \quad\left|\rho_{1}^{\prime}(t)\right| \leq K_{0} \varepsilon^{\kappa} .
$$

Remark. Note that $u$ has to be modulated in order to get the correct result. However, in this case we have not modulated on the scaling and spatial translation parameters because (1-15) is not invariant under these transformations. Nevertheless, we still have another degeneracy, due to time translations, which fortunately allows control of the dynamics of the solution $u$ for every $t \in\left[-T_{\varepsilon}, T_{\varepsilon}\right]$. In this sense, the new time $s(t):=t+\rho_{1}(t)$ can be interpreted as a retarded or advanced time of the actual solution with respect to the approximate solution. Moreover, for $\varepsilon$ small enough, we have

$$
s^{\prime}(t)=1+\rho^{\prime}(t)>\frac{99}{100}>0,
$$

for all $t \in\left[-T_{\varepsilon}, T_{\varepsilon}\right]$. This means that we can invert $s(t)$ on $s\left(\left[-T_{\varepsilon}, T_{\varepsilon}\right]\right) \subseteq \frac{99}{100}\left[-T_{\varepsilon}, T_{\varepsilon}\right]$.

From the proof we do not know the sign of $\rho_{1}^{\prime}(t)$, so in particular we do not know if the solution $u$ is retarded or in advance with respect to the approximate solution $\tilde{u}$. 
Proof of Proposition 5.1. Let $K^{*}>1$ be a constant to be fixed later. Recall from Proposition 2.2 that $u(t)$ is globally well defined in $H^{1}(\mathbb{R})$. Since $\left\|u\left(-T_{\varepsilon}\right)-\tilde{u}\left(-T_{\varepsilon}\right)\right\|_{H^{1}(\mathbb{R})} \leq K \varepsilon^{\kappa}$, by continuity in time in $H^{1}(\mathbb{R})$, we can define $-T_{\varepsilon}<T^{*} \leq T_{\varepsilon}$ by

$$
T^{*}:=\sup \left\{\begin{array}{r}
T \in\left[-T_{\varepsilon}, T_{\varepsilon}\right]: \text { for all } t \in\left[-T_{\varepsilon}, T\right], \text { there exists } r(t) \in \mathbb{R} \\
\text { with }\|u(t+r(t))-\tilde{u}(t)\|_{H^{1}(\mathbb{R})} \leq K^{*} \varepsilon^{\kappa}
\end{array}\right\} .
$$

The goal is to prove that $T^{*}=T_{\varepsilon}$ for $K^{*}$ large enough. To achieve this, we assume otherwise and reach a contradiction with the definition of $T^{*}$ via some independent estimates for $\|u(t+r(t))-\tilde{u}(t)\|_{H^{1}(\mathbb{R})}$ on $\left[-T_{\varepsilon}, T^{*}\right]$, for a special modulation parameter $r(t)$.

Modulation. By using the implicit function theorem we will construct a modulation parameter and to estimate its variation in time:

Lemma 5.2 (Modulation in time). Assume $0<\varepsilon<\varepsilon_{0}\left(K^{*}\right)$ small enough. There exists a unique $C^{1}$ function $\rho_{1}(t)$ such that the function

$$
z(t)=u\left(t+\rho_{1}(t)\right)-\tilde{u}(t)
$$

satisfies, for all $t \in\left[-T_{\varepsilon}, T^{*}\right]$,

$$
\int_{\mathbb{R}} z(t, x) Q_{c}^{\prime}(y) d x=0 .
$$

For all $t \in\left[-T_{\varepsilon}, T^{*}\right]$, we have

$$
\left|\rho_{1}\left(-T_{\varepsilon}\right)\right|+\left\|z\left(-T_{\varepsilon}\right)\right\|_{H^{1}(\mathbb{R})} \leq K \varepsilon^{\kappa}, \quad\|z(t)\|_{H^{1}(\mathbb{R})} \leq 2 K^{*} \varepsilon^{\kappa} .
$$

In addition, $z(t)$ satisfies the equation

$$
z_{t}+\left(1+\rho_{1}^{\prime}\right)\left(z_{x x}-\lambda z+a_{\varepsilon}\left[(\tilde{u}+z)^{m}-\tilde{u}^{m}\right]\right)_{x}-\rho_{1}^{\prime} \tilde{u}_{t}+\left(1+\rho_{1}^{\prime}\right) S[\tilde{u}]=0 .
$$

Finally, there exist $K, \gamma>0$ independent of $K^{*}$ such that for every $t \in\left[-T_{\varepsilon}, T^{*}\right]$

$$
\left|\rho_{1}^{\prime}(t)\right| \leq \frac{K}{c(\varepsilon t)-\lambda}\left(\|z\|_{L^{2}(\mathbb{R})}+\varepsilon e^{-\gamma \varepsilon|t|}\|z(t)\|_{L^{2}(\mathbb{R})}+\|z(t)\|_{L^{2}(\mathbb{R})}^{2}+\|S[\tilde{u}]\|_{L^{2}(\mathbb{R})}\right) .
$$

Proof. The proof of (5-4) and (5-5) is a by now well-known consequence of the implicit function theorem; see, e.g., [Martel and Merle 2007]. The proof of (5-6) follows after a simple calculation using (1-15).

To prove (5-7), we take the time derivative of (5-4) and substitute replace $z_{t}$ from (5-6) to obtain

$$
\begin{aligned}
0=\left(1+\rho_{1}^{\prime}\right) \int_{\mathbb{R}}\left\{z_{x x}-c z+a_{\varepsilon}\left[(\tilde{u}+z)^{m}-\tilde{u}^{m}\right]\right\} Q_{c}^{\prime \prime} & \\
& +\rho_{1}^{\prime} \int_{\mathbb{R}}\left(\tilde{u}_{t}-(c-\lambda) z_{x}\right) Q_{c}^{\prime}-\left(1+\rho_{1}^{\prime}\right) \int_{\mathbb{R}} S[\tilde{u}] Q_{c}^{\prime}+\varepsilon c^{\prime}(\varepsilon t) \int_{\mathbb{R}} z \Lambda Q_{c}^{\prime} .
\end{aligned}
$$

Now note that

$$
\rho_{1}^{\prime} \int_{\mathbb{R}}\left(\tilde{u}_{t}-(c-\lambda) z_{x}\right) Q_{c}^{\prime}=-\frac{\rho_{1}^{\prime}}{\tilde{a}}\left((c-\lambda) \int_{\mathbb{R}} Q_{c}^{\prime 2}+O\left(\varepsilon+\|z(t)\|_{L^{2}(\mathbb{R})}\right)\right) .
$$

On the other hand,

$$
\int_{\mathbb{R}}\left(z_{x x}-c z+a_{\varepsilon}\left[(\tilde{u}+z)^{m}-\tilde{u}^{m}\right]\right) Q_{c}^{\prime \prime}=-\int_{\mathbb{R}} z \mathscr{L} Q_{c}^{\prime \prime}+O\left(\varepsilon e^{-\gamma \varepsilon|t|}\|z(t)\|_{L^{2}(\mathbb{R})}\right)+O\left(\|z(t)\|_{L^{2}(\mathbb{R})}^{2}\right) .
$$


Collecting these estimates and using the fact that $\|z(t)\|_{H^{1}(\mathbb{R})}$ is small, we get desired result.

Control in the $\boldsymbol{Q}_{\boldsymbol{c}}$ direction. We recall from (1-7) that the energy of the function $u\left(t+\rho_{1}(t)\right)$ is conserved; moreover, $E_{a}\left[u\left(t+\rho_{1}(t)\right)\right]=E_{a}[u](t)$ for any $t \in\left[-T_{\varepsilon}, T^{*}\right]$. In what follows, we will made use of this identity to estimate $z$ against the degenerate direction $Q_{c}$. First we prove that the approximate solution $\tilde{u}$ has almost conserved energy.

Lemma 5.3 (Almost conservation of energy). For the approximate solution ũ from Proposition 4.7,

$$
\partial_{t} E_{a}[\tilde{u}](t)=-\int_{\mathbb{R}}\left(\tilde{u}_{x x}-\lambda \tilde{u}+a_{\varepsilon} \tilde{u}^{m}\right) S[\tilde{u}] .
$$

In particular, there exists $K>0$ independent of $K^{*}$ such that

$$
\left|E_{a}[\tilde{u}](t)-E_{a}[\tilde{u}]\left(-T_{\varepsilon}\right)\right| \leq K \varepsilon^{\kappa} .
$$

Proof. From (4-9) we have

$$
\begin{aligned}
\int_{\mathbb{R}} S[\tilde{u}]\left(\tilde{u}_{x x}-\lambda \tilde{u}+a_{\varepsilon} \tilde{u}^{m}\right) & =\int_{\mathbb{R}} \tilde{u}_{t}\left(\tilde{u}_{x x}-\lambda \tilde{u}+a_{\varepsilon} \tilde{u}^{m}\right) \\
& =-\partial_{t} \frac{1}{2} \int_{\mathbb{R}} \tilde{u}_{x}^{2}-\partial_{t} \frac{\lambda}{2} \int_{\mathbb{R}} \tilde{u}^{2}+\frac{1}{m+1} \partial_{t} \int_{\mathbb{R}} a_{\varepsilon} \tilde{u}^{m+1}=-\partial_{t} E_{a}[\tilde{u}](t),
\end{aligned}
$$

which shows (5-8). For (5-9), we have, from the Cauchy-Schwarz inequality,

$$
\left|\partial_{t} E_{a}[\tilde{u}](t)\right| \leq K\|S[\tilde{u}](t)\|_{L^{2}(\mathbb{R})},
$$

for some constant $K>0$. After integrating and considering (5-1), we get the result.

Lemma 5.4 (Control in the $Q_{c}$ direction). There exist $K, \gamma>0$ independent of $K^{*}$ and such that, for $0<\varepsilon<\varepsilon_{0}$ small enough,

$$
\left|\int_{\mathbb{R}} Q_{c}(y) z\right| \leq \frac{K}{c(\varepsilon t)-\lambda}\left(\varepsilon^{\kappa}+\varepsilon^{1 / 2} e^{-\varepsilon \gamma|t|}\|z(t)\|_{L^{2}(\mathbb{R})}+\|z(t)\|_{H^{1}(\mathbb{R})}^{2}\right) .
$$

Proof. We expand the expression of the conserved energy $E_{a}\left[u\left(t+\rho_{1}\right)\right]$ and make use of the identity $u\left(t+\rho_{1}\right)=\tilde{u}(t)+z(t)$ to obtain

$$
\begin{aligned}
E_{a}[\tilde{u}+z](t)=E_{a}[\tilde{u}](t)-\int_{\mathbb{R}} z\left(\tilde{u}_{x x}-\lambda \tilde{u}+a_{\varepsilon} \tilde{u}^{m}\right) & +\frac{1}{2} \int_{\mathbb{R}} z_{x}^{2}+\frac{\lambda}{2} \int_{\mathbb{R}} z^{2} \\
& -\frac{1}{m+1} \int_{\mathbb{R}} a_{\varepsilon}\left((\tilde{u}+z)^{m+1}-\tilde{u}^{m+1}-(m+1) \tilde{u}^{m} z\right) .
\end{aligned}
$$

Note that

$$
\int_{\mathbb{R}} z\left(\tilde{u}_{x x}-\lambda \tilde{u}+a_{\varepsilon} \tilde{u}^{m}\right)(t)=\int_{\mathbb{R}} z\left(\tilde{u}_{x x}-\lambda \tilde{u}+a_{\varepsilon} \tilde{u}^{m}\right)\left(-T_{\varepsilon}\right)+\left(E_{a}[\tilde{u}](t)-E_{a}[\tilde{u}]\left(-T_{\varepsilon}\right)\right)+O\left(\|z(t)\|_{H^{1}(\mathbb{R})}^{2}\right) .
$$

We now use (4-32):

$$
\int_{\mathbb{R}} z\left(\tilde{u}_{x x}-\lambda u+a_{\varepsilon} \tilde{u}^{m}\right)=(c-\lambda) \int_{\mathbb{R}} \tilde{u} z+O\left(\varepsilon e^{-\gamma \varepsilon|t|}\|z(t)\|_{L^{2}(\mathbb{R})}\right) .
$$

The conclusion follows from this identity and (5-9). 
Energy functional for $z$. Consider the functional

$$
\mathscr{F}(t):=\frac{1}{2} \int_{\mathbb{R}}\left(z_{x}^{2}+c(\varepsilon t) z^{2}\right)-\frac{1}{m+1} \int_{\mathbb{R}} a_{\varepsilon}\left((\tilde{u}+z)^{m+1}-\tilde{u}^{m+1}-(m+1) \tilde{u}^{m} z\right) .
$$

Lemma 5.5 (Modified coercivity for $\mathscr{F}$ ). There exist $K, v_{0}>0$, independent of $K^{*}$ and $\varepsilon$, such that, for every $t \in\left[-T_{\varepsilon}, T_{\varepsilon}\right]$,

$$
\mathscr{F}_{(}(t) \geq v_{0}\|z(t)\|_{H^{1}(\mathbb{R})}^{2}-\left|\int_{\mathbb{R}} Q_{c}(y) z\right|^{2}-K\left(\varepsilon e^{-\gamma \varepsilon|t|}+\varepsilon^{2}\right)\|z(t)\|_{L^{2}(\mathbb{R})}^{2}-K\|z(t)\|_{L^{2}(\mathbb{R})}^{3} .
$$

Proof. We write $\mathscr{F}(t)$ as the sum of

$$
\frac{1}{2} \int_{\mathbb{R}}\left(z_{x}^{2}+c z^{2}-m a_{\varepsilon} \tilde{u}^{m-1} z^{2}\right)
$$

and

$$
-\frac{1}{m+1} \int_{\mathbb{R}} a_{\varepsilon}\left((\tilde{u}+z)^{m+1}-\tilde{u}^{m+1}-(m+1) \tilde{u}^{m} z-\frac{1}{2} m(m+1) \tilde{u}^{m-1} z^{2}\right) .
$$

In the case $m=2$ the term (5-12) is identically zero, and for $m=3,4$ we have $|(5-12)| \leq K\|z(t)\|_{L^{2}(\mathbb{R})}^{3}$. The other summand is

$$
(5-11)=\frac{1}{2} \int_{\mathbb{R}}\left(z_{x}^{2}+c(\varepsilon t) z^{2}-m Q_{c}^{m-1} z^{2}\right)-\varepsilon \frac{m a^{\prime}(\varepsilon \rho)}{2 a(\varepsilon \rho)} \int_{\mathbb{R}} y Q_{c}^{m-1} z^{2}+O\left(\varepsilon^{2}\|z(t)\|_{L^{2}(\mathbb{R})}^{2}\right) .
$$

It is clear that

$$
\left|\varepsilon \frac{m a^{\prime}(\varepsilon \rho)}{2 a(\varepsilon \rho)} \int_{\mathbb{R}} y Q_{c}^{m-1} z^{2}\right| \leq K \varepsilon e^{-\gamma \varepsilon|t|}\|z(t)\|_{L^{2}(\mathbb{R})}^{2} .
$$

Finally, by Lemma 2.3, there exist constants $K, v_{0}>0$ such that, for all $t \in\left[-T_{\varepsilon}, T^{*}\right]$,

$$
\frac{1}{2} \int_{\mathbb{R}}\left(z_{x}^{2}+c(\varepsilon t) z^{2}-m Q_{c}^{m-1} z^{2}\right) \geq v_{0}\|z(t)\|_{H^{1}(\mathbb{R})}^{2}-K\left|\int_{\mathbb{R}} Q_{c} z\right|^{2} .
$$

Now we use a coercivity argument to obtain independent estimates for $\mathscr{F}\left(T^{*}\right)$.

Lemma 5.6 (Estimates on $\left.\mathscr{F}\left(T^{*}\right)\right)$. The following properties hold for any $t \in\left[-T_{\varepsilon}, T^{*}\right]$.

(1) First time derivative.

$\mathscr{F}^{\prime}(t)=-\int_{\mathbb{R}} z_{t}\left(z_{x x}-c z+a_{\varepsilon}\left((\tilde{u}+z)^{m}-\tilde{u}^{m}\right)\right)+\frac{1}{2} \varepsilon c^{\prime}(\varepsilon t) \int_{\mathbb{R}} z^{2}-\int_{\mathbb{R}} a_{\varepsilon} \tilde{u}_{t}\left((\tilde{u}+z)^{m}-\tilde{u}^{m}-m \tilde{u}^{m-1} z\right)$.

(2) Integration in time. There exist constants $K, \gamma>0$ such that $\mathscr{F}(t)-\mathscr{F}\left(-T_{\varepsilon}\right)$

$$
\leq K\left(K^{*}\right)^{4} \varepsilon^{4 \kappa-\frac{1}{100}}+K\left(K^{*}\right)^{3} \varepsilon^{3 \kappa-\frac{1}{100}}+K K^{*} \varepsilon^{2 \kappa}+K \int_{-T_{\varepsilon}}^{t} \varepsilon e^{-\varepsilon \gamma|t|}\|z(t)\|_{H^{1}(\mathbb{R})}^{2} d t .
$$


Proof. Statement (1) amounts to a simple computation. Let us consider (5-13). Substituting (5-6) into the equality in (1) we can write $\mathscr{F}^{\prime}(t)$ as a sum of the four terms

$$
\begin{aligned}
& (c(\varepsilon t)-\lambda)\left(1+\rho_{1}^{\prime}\right) \int_{\mathbb{R}} a_{\varepsilon}\left((\tilde{u}+z)^{m}-\tilde{u}^{m}\right) z_{x}, \\
& -\rho_{1}^{\prime} \int_{\mathbb{R}} \tilde{u}_{t}\left(z_{x x}-c z+a_{\varepsilon}\left((\tilde{u}+z)^{m}-\tilde{u}^{m}\right)\right), \\
& \left(1+\rho_{1}^{\prime}\right) \int_{\mathbb{R}} S[\tilde{u}]\left(z_{x x}-c z+a_{\varepsilon}\left((\tilde{u}+z)^{m}-\tilde{u}^{m}\right)\right), \\
& \frac{1}{2} \varepsilon c^{\prime}(\varepsilon t) \int_{\mathbb{R}} z^{2}-\int_{\mathbb{R}} a_{\varepsilon} \tilde{u}_{t}\left((\tilde{u}+z)^{m}-\tilde{u}^{m}-m \tilde{u}^{m-1} z\right) .
\end{aligned}
$$

We consider first the case $m=2$. After some simplifications, we get for (5-14) the value

$$
(c-\lambda)\left(1+\rho_{1}^{\prime}\right) \int_{\mathbb{R}} a_{\varepsilon}\left(2 \tilde{u} z+z^{2}\right) z_{x}=-(c-\lambda)\left(1+\rho_{1}^{\prime}\right) \int_{\mathbb{R}}\left(a_{\varepsilon} \tilde{u}_{x} z^{2}+\varepsilon a^{\prime}(\varepsilon x) \tilde{u} z^{2}+\frac{1}{3} \varepsilon a^{\prime}(\varepsilon x) z^{3}\right) .
$$

Hence

$$
\left|(5-14)+(c-\lambda)\left(1+\rho_{1}^{\prime}\right) \int_{\mathbb{R}} a_{\varepsilon} \tilde{u}_{x} z^{2}\right| \leq K \varepsilon e^{-\gamma \varepsilon|t|}\|z(t)\|_{L^{2}(\mathbb{R})}^{2}+K \varepsilon\|z(t)\|_{H^{1}(\mathbb{R})}^{3} .
$$

On the other hand,

$$
\begin{aligned}
(5-15)=-\rho_{1}^{\prime} \int_{\mathbb{R}}\left(\tilde{u}_{t}+(c-\lambda) \tilde{u}_{x}\right)\left(z_{x x}\right. & \left.-c z+a_{\varepsilon}\left(2 \tilde{u} z+z^{2}\right)\right)+(c-\lambda) \rho_{1}^{\prime} \int_{\mathbb{R}} a_{\varepsilon} \tilde{u}_{x} z^{2} \\
& +(c-\lambda) \rho_{1}^{\prime} \int_{\mathbb{R}} z\left(\tilde{u}_{x x}-c \tilde{u}+a_{\varepsilon} \tilde{u}^{2}\right)_{x}-(c-\lambda) \rho_{1}^{\prime} \varepsilon \int_{\mathbb{R}} a^{\prime}(\varepsilon x) \tilde{u}^{2} z .
\end{aligned}
$$

Using the estimates (4-30), (4-33) and (4-31) we then obtain

$$
\left|(5-15)-(c-\lambda) \rho_{1}^{\prime} \int_{\mathbb{R}} a_{\varepsilon} \tilde{u}_{x} z^{2}\right| \leq K \varepsilon\left|\rho_{1}^{\prime}\right| e^{-\gamma \varepsilon|t|}\|z(t)\|_{H^{1}(\mathbb{R})} .
$$

We also have

$$
(5-16)=\left(1+\rho_{1}^{\prime}\right) \int_{\mathbb{R}} z\left(S[\tilde{u}]_{x x}-c S[\tilde{u}]+2 a_{\varepsilon} \tilde{u} S[\tilde{u}]+a_{\varepsilon} z S[\tilde{u}]\right)
$$

thus using (5-7)

$$
|(5-16)| \leq K\|z(t)\|_{L^{2}(\mathbb{R})}\|S[\tilde{u}](t)\|_{H^{2}(\mathbb{R})} .
$$

Finally,

$$
(5-17)=\frac{1}{2} \varepsilon c^{\prime}(\varepsilon t) \int_{\mathbb{R}} z^{2}-\int_{\mathbb{R}} a_{\varepsilon}\left(\tilde{u}_{t}+(c-\lambda) \tilde{u}_{x}\right) z^{2}+(c-\lambda) \int_{\mathbb{R}} a_{\varepsilon} \tilde{u}_{x} z^{2} .
$$

We get then from (4-31)

$$
\left|(5-17)-(c-\lambda) \int_{\mathbb{R}} a_{\varepsilon} \tilde{u}_{x} z^{2}\right| \leq K \varepsilon e^{-\gamma \varepsilon|t|}\|z(t)\|_{L^{2}(\mathbb{R})}^{2} .
$$

Collecting these estimates and (5-7), we obtain, after an integration,

$$
\left|\mathscr{F}(t)-\mathscr{F}\left(-T_{\varepsilon}\right)\right| \leq K\left(K^{*}\right)^{3} \varepsilon^{3 \kappa-\frac{1}{100}}+K K^{*} \varepsilon^{2 \kappa}+K \int_{-T_{c}}^{t} \varepsilon e^{-\gamma \varepsilon|s|}\|z(s)\|_{L^{2}(\mathbb{R})}^{2} d s .
$$


The cases $m=3,4$ are similar, but more involved. From (5-14)-(5-17), and after some integration by parts, we obtain for $\mathscr{F}^{\prime}(t)$ the expression

$$
\begin{aligned}
& (c-\lambda)\left(1+\rho_{1}^{\prime}\right)\left(\int_{\mathbb{R}} a_{\varepsilon}\left((\tilde{u}+z)^{m}-\tilde{u}^{m}-m \tilde{u}^{m-1} z-\frac{m}{2}(m-1) \tilde{u}^{m-2} z^{2}\right) z_{x}\right. \\
& \left.-\frac{m}{2} \varepsilon \int_{\mathbb{R}} a^{\prime}(\varepsilon x) \tilde{u}^{m-1} z^{2}-\frac{\varepsilon}{6} m(m-1) \int_{\mathbb{R}} a^{\prime}(\varepsilon x) \tilde{u}^{m-2} z^{3}-\frac{m}{2} \int_{\mathbb{R}} a_{\varepsilon}\left(\tilde{u}^{m-1}\right)_{x} z^{2}-\frac{m}{6}(m-1) \int_{\mathbb{R}} a_{\varepsilon}\left(\tilde{u}^{m-2}\right)_{x} z^{3}\right) \\
& -\rho_{1}^{\prime} \int_{\mathbb{R}}\left(\tilde{u}_{t}+(c-\lambda) \tilde{u}_{x}\right)\left(z_{x x}-c z+a_{\varepsilon}\left((\tilde{u}+z)^{m}-\tilde{u}^{m}\right)\right)+(c-\lambda) \rho_{1}^{\prime}\left(\int_{\mathbb{R}} z\left(\tilde{u}_{x x}-c \tilde{u}+a_{\varepsilon} \tilde{u}^{m}\right)_{x}-\varepsilon \int_{\mathbb{R}} a^{\prime}(\varepsilon x) \tilde{u}^{m} z\right) \\
& +(c-\lambda)\left(1+\rho_{1}^{\prime}\right) \int_{\mathbb{R}} \tilde{u}_{x} a_{\varepsilon}\left((\tilde{u}+z)^{m}-\tilde{u}^{m}-m \tilde{u}^{m-1} z-\frac{m}{2}(m-1) \tilde{u}^{m-2} z^{2}-\frac{m}{6}(m-1)(m-2) \tilde{u}^{m-3} z^{3}\right) \\
& +\frac{m}{2}(c-\lambda) \rho_{1}^{\prime}\left(\int_{\mathbb{R}} a_{\varepsilon}\left(\tilde{u}^{m-1}\right)_{x} z^{2}+\frac{m-1}{3} \int_{\mathbb{R}} a_{\varepsilon}\left(\tilde{u}^{m-2}\right)_{x} z^{3}\right) \\
& +\left(1+\rho_{1}^{\prime}\right) \int_{\mathbb{R}} z\left(S[\tilde{u}]_{x x}-c S[\tilde{u}]+m a_{\varepsilon} \tilde{u}^{m-1} S[\tilde{u}]\right)+\left(1+\rho_{1}^{\prime}\right) \int_{\mathbb{R}} a_{\varepsilon}\left((\tilde{u}+z)^{m}-\tilde{u}^{m}-m \tilde{u}^{m-1} z\right) S[\tilde{u}] \\
& +\frac{\varepsilon}{2} c^{\prime} \int_{\mathbb{R}} z^{2}-\int_{\mathbb{R}} a_{\varepsilon}\left(\tilde{u}_{t}+(c-\lambda) \tilde{u}_{x}\right)\left((\tilde{u}+z)^{m}-\tilde{u}^{m}-m \tilde{u}^{m-1} z\right) \\
& +\frac{m}{2}(c-\lambda)\left(\int_{\mathbb{R}} a_{\varepsilon}\left(\tilde{u}^{m-1}\right)_{x} z^{2}+\frac{m-1}{3} \int_{\mathbb{R}} a_{\varepsilon}\left(\tilde{u}^{m-2}\right)_{x} z^{3}\right) .
\end{aligned}
$$

Note that the last two terms in (5-19) disappear, as do (5-21) and (5-22). With (5-18) and (5-20), we need a little more care. For $m=3$,

$$
|(5-18)+(5-20)|=\left|\frac{1}{4} \varepsilon(c-\lambda)\left(1+\rho_{1}^{\prime}\right) \int_{\mathbb{R}} a^{\prime}(\varepsilon x) z^{4}\right| \leq \varepsilon\|z(t)\|_{L^{2}(\mathbb{R})}^{4} .
$$

In the case $m=4$,

$$
\begin{aligned}
(5-18)+(5-20) & =(c-\lambda)\left(1+\rho_{1}^{\prime}\right) \int_{\mathbb{R}} a_{\varepsilon}\left[z_{x}\left(4 \tilde{u} z^{3}+z^{4}\right)+\tilde{u}_{x} z^{4}\right] \\
& =-\varepsilon(c-\lambda)\left(1+\rho_{1}^{\prime}\right) \int_{\mathbb{R}} a^{\prime}(\varepsilon x)\left(\tilde{u} z^{4}+z^{5}\right) .
\end{aligned}
$$

Consequently we have

$$
|(5-18)+(5-20)| \leq K \varepsilon e^{-\gamma \varepsilon|t|}\|z(t)\|_{L^{2}(\mathbb{R})}^{4}+K \varepsilon\|z(t)\|_{L^{2}(\mathbb{R})}^{5} .
$$

Finally, using (4-30), (4-33), (4-31) we obtain

$$
\begin{aligned}
\mathscr{F}^{\prime}(t) \leq K \varepsilon\|z(t)\|_{H^{1}(\mathbb{R})}^{4}+K \varepsilon e^{-\gamma \varepsilon|t|}\|z(t)\|_{L^{2}(\mathbb{R})}^{2}+K \varepsilon\|z(t)\|_{H^{1}(\mathbb{R})}^{3} & \\
& +K\left|\rho_{1}^{\prime}(t)\right| \varepsilon e^{-\gamma \varepsilon|t|}\|z(t)\|_{H^{1}(\mathbb{R})}+K\|S[\tilde{u}](t)\|_{H^{2}(\mathbb{R})}\|z(t)\|_{L^{2}(\mathbb{R})}
\end{aligned}
$$

Integrating and using (5-7), we obtain

$$
\mathscr{F}(t)-\mathscr{F}\left(-T_{\varepsilon}\right) \leq K\left(K^{*}\right)^{4} \varepsilon^{4 \kappa-\frac{1}{100}}+K\left(K^{*}\right)^{3} \varepsilon^{3 \kappa-\frac{1}{100}}+K K^{*} \varepsilon^{2 \kappa}+K \int_{-T_{\varepsilon}}^{t} \varepsilon e^{-\gamma \varepsilon|s|}\|z(s)\|_{H^{1}(\mathbb{R})}^{2} d s,
$$

completing the proof. 
We are finally in a position to show that $T^{*}<T_{\varepsilon}$ leads to a contradiction.

End of proof of Proposition 5.1. From Lemma 5.2, $\mathscr{F}\left(-T_{\varepsilon}\right) \leq K c^{2 \kappa}$, and from Lemmas 5.5, 5.4 and (5-13) we get

$$
\begin{aligned}
&\|z(t)\|_{L^{2}(\mathbb{R}) \leq}^{2} \leq K\left|\int_{\mathbb{R}} z Q_{c}(y)\right|^{2}+K \varepsilon^{2 \kappa}+K\left(K^{*}\right)^{4} \varepsilon^{4 \kappa-\frac{1}{100}}+K\left(K^{*}\right)^{3} \varepsilon^{3 \kappa-\frac{1}{100}}+K K^{*} \varepsilon^{2 \kappa}+K \int_{-T_{\varepsilon}}^{t} \varepsilon e^{-\gamma \varepsilon|t|}\|z(t)\|_{L^{2}(\mathbb{R})}^{2} d t \\
& \leq K\left|\varepsilon^{\kappa}+K^{*} \varepsilon^{\frac{1}{2}+\kappa} e^{-\gamma \varepsilon|t|}+\left(K^{*}\right)^{2} \varepsilon^{2 \kappa}+\|S[\tilde{u}]\|_{L^{2}(\mathbb{R})}\right|^{2}+K \varepsilon^{2 \kappa} \\
&+K\left(K^{*}\right)^{4} \varepsilon^{4 \kappa-\frac{1}{100}}+K\left(K^{*}\right)^{3} \varepsilon^{3 \kappa-\frac{1}{100}}+K K^{*} \varepsilon^{2 \kappa}+K \int_{-T_{\varepsilon}}^{t} \varepsilon e^{-\gamma \varepsilon|s|}\|z(s)\|_{L^{2}(\mathbb{R})}^{2} d s \\
& \leq K \varepsilon^{2 \kappa}+K\left(K^{*}\right)^{4} \varepsilon^{4 \kappa-\frac{1}{100}}+K\left(K^{*}\right)^{3} \varepsilon^{3 \kappa-\frac{1}{100}}+K K^{*} \varepsilon^{2 \kappa}+K \int_{-T_{\varepsilon}}^{t} \varepsilon e^{-\gamma \varepsilon|s|}\|z(s)\|_{L^{2}(\mathbb{R})}^{2} d s .
\end{aligned}
$$

Using Gronwall's inequality (see [Verhulst 2006], for instance) we conclude that, for some large constant $K>0$ independent of $K^{*}$ and $\varepsilon$,

$$
\|z(t)\|_{H^{1}(\mathbb{R})}^{2} \leq K \varepsilon^{2 \kappa}+K\left(K^{*}\right)^{4} \varepsilon^{4 \kappa-\frac{1}{100}}+K\left(K^{*}\right)^{3} \varepsilon^{3 \kappa-\frac{1}{100}}+K K^{*} \varepsilon^{2 \kappa} .
$$

Taking $\varepsilon$ small and $K^{*}$ large enough, we then obtain that for all $t \in\left[-T_{\varepsilon}, T^{*}\right]$,

$$
\|z(t)\|_{H^{1}(\mathbb{R})}^{2} \leq \frac{1}{2}\left(K^{*}\right)^{2} \varepsilon^{2 \kappa} .
$$

This contradicts the definition of $T^{*}$ and concludes the proof of Proposition 5.1.

Proof of Theorem 4.1. We are now able to prove Theorem 4.1, showing that the solution $u(t)$ given by Theorem 3.1 is close to the approximate solution $\tilde{u}(t)$ constructed in Proposition 4.7 at time $t=-T_{\varepsilon}$. Behavior at $t=-T_{\varepsilon}$. From (3-3), Proposition 4.9, and more specifically (4-34) we have

$$
\left\|u\left(-T_{\varepsilon}\right)-\tilde{u}\left(-T_{\varepsilon}\right)\right\|_{H^{1}(\mathbb{R})} \leq K \varepsilon^{10} .
$$

Behavior at $t=T_{\varepsilon}$. Thanks to the above estimate and (4-29) we can invoke Proposition 5.1 with $\kappa:=\frac{1}{2}$ to obtain the existence of $K_{0}, \varepsilon_{0}>0$ such that for all $0<\varepsilon<\varepsilon_{0}$

$$
\left\|u\left(T_{\varepsilon}+\rho_{1}\left(T_{\varepsilon}\right)\right)-\tilde{u}\left(T_{\varepsilon}\right)\right\|_{H^{1}(\mathbb{R})} \leq K_{0} \varepsilon^{1 / 2}, \quad\left|\rho_{1}\left(T_{\varepsilon}\right)\right| \leq K_{0} \varepsilon^{-\frac{1}{2}-\frac{1}{100}} \leq \frac{T_{\varepsilon}}{100} .
$$

Therefore from (4-35) and the triangle inequality,

$$
\left\|u\left(T_{\varepsilon}+\rho_{1}\left(T_{\varepsilon}\right)\right)-2^{-1 /(m-1)} Q_{c_{\infty}}\left(\cdot-\rho\left(T_{\varepsilon}\right)\right)\right\|_{H^{1}(\mathbb{R})} \leq K_{0} \varepsilon^{1 / 2} .
$$

(see also (4-5).) Finally note that $(1-\lambda) T_{\varepsilon} \leq \rho\left(T_{\varepsilon}\right) \leq\left(2 c_{\infty}(\lambda)-\lambda-1\right) T_{\varepsilon}$. This finishes the proof.

\section{Asymptotics for large times}

Recall that for large times $\left(t \geq T_{\varepsilon}\right)$ the soliton-like solution is expected to be far away from the region where $a_{\varepsilon}$ varies. Roughly speaking, the solution's stability and asymptotic stability properties will follow 
from the fact that in this region (1-13) the equation behaves like the $\mathrm{gKdV}$ equation

$$
u_{t}+\left(u_{x x}-\lambda u+2 u^{m}\right)_{x}=0 \quad \text { in }\left\{t \geq T_{\varepsilon}\right\} \times \mathbb{R}_{x}
$$

The purpose of this section is to lay out this argument in a rigorous way. We start by restating the asymptotic behavior, already described in Theorem 1.2. Recall the parameters $\lambda_{0}$ and $c_{\infty}(\lambda)$ from Theorems 1.1 and 4.1 .

Theorem 6.1 (Stability and asymptotic stability in $H^{1}$ ). Suppose $m=2,4$ with $0<\lambda \leq \lambda_{0}$, or $m=3$ with $0 \leq \lambda \leq \lambda_{0}$. Let $0<\beta<\frac{1}{2}\left(c_{\infty}(\lambda)-\lambda\right)$. There exists $\varepsilon_{0}>0$ such that if $0<\varepsilon<\varepsilon_{0}$ the following statements hold. Suppose that for some time $t_{1} \geq \frac{1}{2} T_{\varepsilon}$ with $t_{1} \leq X_{0} \leq 2 t_{1}$ we have

$$
\left\|u\left(t_{1}\right)-2^{-1 /(m-1)} Q_{c_{\infty}}\left(x-X_{0}\right)\right\|_{H^{1}(\mathbb{R})} \leq \varepsilon^{1 / 2},
$$

where $u(t)$ is an $H^{1}$-solution of (1-15). Then $u(t)$ is defined for every $t \geq t_{1}$ and there exists $K, c^{+}>0$ and a $C^{1}$-function $\rho_{2}(t)$ defined in $\left[t_{1},+\infty\right)$ with these properties:

(1) Stability.

$$
\sup _{t \geq t_{1}}\left\|u(t)-2^{-1 /(m-1)} Q_{c_{\infty}}\left(\cdot-\rho_{2}(t)\right)\right\|_{H^{1}(\mathbb{R})} \leq K \varepsilon^{1 / 2}
$$

where

$$
\left|\rho_{2}\left(t_{1}\right)-X_{0}\right| \leq K \varepsilon^{1 / 2} \quad \text { and } \quad\left|\rho_{2}^{\prime}(t)-c_{\infty}(\lambda)+\lambda\right| \leq K \varepsilon^{1 / 2} \text { for all } t \geq t_{1} \text {. }
$$

(2) Asymptotic stability.

$$
\lim _{t \rightarrow+\infty}\left\|u(t)-2^{-1 /(m-1)} Q_{c^{+}}\left(\cdot-\rho_{2}(t)\right)\right\|_{H^{1}(x>\beta t)}=0 .
$$

In addition,

$$
\lim _{t \rightarrow+\infty} \rho_{2}^{\prime}(t)=c^{+}-\lambda, \quad\left|c^{+}-c_{\infty}\right| \leq K \varepsilon^{1 / 2}
$$

Remarks. (a) We do not know if the stability results are valid in the cases $m=2,4$ and $\lambda=0$. Clearly, the stability property as stated above is false if $\limsup _{t \rightarrow+\infty}\|u(t)\|_{L^{2}(\mathbb{R})}=+\infty$.

(b) For $0<\lambda<\lambda_{0}$ the asymptotic stability property (6-3) holds for any $\beta>-\lambda$, provided $\varepsilon_{0}$ is small enough. We make use of this property in $[\mathrm{Muñoz} \geq 2011 \mathrm{a}]$, but we do not pursue it here.

Proof of Theorem 6.1(1): stability. The proof of stability is standard and similar to that of Proposition 5.1. For this reason we omit many details, inviting the reader to consult [Benjamin 1972; Martel et al. 2002], where the proof originates. Even then the argument will occupy us until page 613.

Assume that, for some fixed $K>0$,

$$
\left\|u\left(t_{1}\right)-2^{-1 /(m-1)} Q_{c_{\infty}}\left(\cdot-X_{0}\right)\right\|_{H^{1}(\mathbb{R})} \leq K \varepsilon^{1 / 2} .
$$

From the local and global Cauchy theory exposed in Proposition 2.1 and Theorems 3.1 and 4.1, we know that the solution $u$ is well defined for all $t \geq t_{1}$. 
To simplify the calculations, note that from (1-18) the function $v:=2^{1 /(m-1)} u$ solves

$$
v_{t}+\left(v_{x x}-\lambda v+\frac{1}{2} a_{\varepsilon} v^{m}\right)_{x}=0 \quad \text { on } \mathbb{R}_{t} \times \mathbb{R}_{x}
$$

Then (6-5) becomes

$$
\left\|v\left(t_{1}\right)-Q_{c_{\infty}}\left(\cdot-X_{0}\right)\right\|_{H^{1}(\mathbb{R})} \leq \tilde{K} \varepsilon^{1 / 2}
$$

With a slight abuse of notation we will rename $v:=u$ and $\tilde{K}:=K$, and we will assume the validity of (6-6) for $u$. The parameters $X_{0}$ and $c_{\infty}$ remain unchanged.

Let $D_{0}>2 \mathrm{~K}$ be a large number to be chosen later, and set

$$
T^{*}:=\sup \left\{\begin{array}{r}
t \geq t_{1}: \forall t^{\prime} \in\left[t_{1}, t\right) \text { there is a smooth } \tilde{\rho}_{2}\left(t^{\prime}\right) \in \mathbb{R} \text { with }\left|\tilde{\rho}_{2}^{\prime}\left(t^{\prime}\right)-c_{\infty}+\lambda\right| \leq \frac{1}{100}, \\
\left|\tilde{\rho}_{2}\left(t_{1}\right)-X_{0}\right| \leq \frac{1}{100}, \text { and }\left\|u\left(t^{\prime}\right)-Q_{c_{\infty}}\left(\cdot-\tilde{\rho}_{2}\left(t^{\prime}\right)\right)\right\|_{H^{1}(\mathbb{R})} \leq D_{0} \varepsilon^{1 / 2}
\end{array}\right\} .
$$

Observe that $T^{*}>t_{1}$ is well defined since $D_{0}>2 \mathrm{~K}$ and because of (6-5) and the continuity of $t \mapsto u(t)$ in $H^{1}(\mathbb{R})$. The goal is to prove $T^{*}=+\infty$, and thus $(6-2)$.

Therefore, for the sake of contradiction, suppose $T^{*}<+\infty$. Using modulation theory around the soliton, we will decompose the solution on $\left[t_{1}, T^{*}\right]$, and so find a special $\rho_{2}(t)$ satisfying the hypotheses in (6-7), but with

$$
\sup _{t \in\left[t_{1}, T^{*}\right]}\left\|u(t)-Q_{c_{\infty}}\left(\cdot-\rho_{2}(t)\right)\right\|_{H^{1}(\mathbb{R})} \leq \frac{1}{2} D_{0} \varepsilon^{1 / 2}
$$

in contradiction with the definition of $T^{*}$.

Lemma 6.2 (Modulated decomposition). For $\varepsilon>0$ small enough, independent of $T^{*}$, there exist $C^{1}$ functions $\rho_{2}, c_{2}$, defined on $\left[t_{1}, T^{*}\right]$, with $c_{2}(t)>0$, such that the function $z(t)$ given by

$$
z(t, x):=u(t, x)-R(t, x)
$$

where $R(t, x):=Q_{c_{2}(t)}\left(x-\rho_{2}(t)\right)$, satisfies the following conditions for all $t \in\left[t_{1}, T^{*}\right]$ :

$$
\begin{gathered}
\int_{\mathbb{R}} R(t, x) z(t, x) d x=\int_{\mathbb{R}}\left(x-\rho_{2}(t)\right) R(t, x) z(t, x) d x=0 \quad \text { (orthogonality), } \\
\|z(t)\|_{H^{1}(\mathbb{R})}+\left|c_{2}(t)-c_{\infty}\right| \leq K D_{0} \varepsilon^{1 / 2}, \\
\left\|z\left(t_{1}\right)\right\|_{H^{1}(\mathbb{R})}+\left|\rho_{2}\left(t_{1}\right)-X_{0}\right|+\left|c_{2}\left(t_{1}\right)-c_{\infty}\right| \leq K \varepsilon^{1 / 2},
\end{gathered}
$$

where $K$ does not depend on $D_{0}$. In addition, $z(t)$ now satisfies the modified $g K d V$ equation

$$
\begin{aligned}
z_{t}+\left(z_{x x}-\lambda z+\frac{1}{2} a_{\varepsilon}\left((R+z)^{m}-R^{m}\right)+\left(\frac{1}{2} a_{\varepsilon}(x)-1\right) Q_{c_{2}}^{m}\right)_{x} & \\
& +c_{2}^{\prime}(t) \Lambda Q_{c_{2}}+\left(c_{2}-\lambda-\rho_{2}^{\prime}\right)(t) Q_{c_{2}}^{\prime}=0 .
\end{aligned}
$$

Furthermore, for some constant $\gamma>0$ independent of $\varepsilon$, we have the improved estimates $\left|\rho_{2}^{\prime}(t)+\lambda-c_{2}(t)\right|$

$$
\leq K(m-3)\left(\int_{\mathbb{R}} e^{-\gamma\left|x-\rho_{2}(t)\right|} z^{2}(t, x) d x\right)^{\frac{1}{2}}+K \int_{\mathbb{R}} e^{-\gamma\left|x-\rho_{2}(t)\right|} z^{2}(t, x) d x+K e^{-\gamma \varepsilon t}
$$


and

$$
\frac{\left|c_{2}^{\prime}(t)\right|}{c_{2}(t)} \leq K \int_{\mathbb{R}} e^{-\gamma\left|x-\rho_{2}(t)\right|} z^{2}(t, x) d x+K e^{-\gamma \varepsilon t}\|z(t)\|_{H^{1}(\mathbb{R})}+K \varepsilon e^{-\varepsilon \gamma t} .
$$

Remark. From (6-11) and taking $\varepsilon$ small enough we have an improved the bound on $\rho_{2}(t)$. Indeed, for all $t \in\left[t_{1}, T^{*}\right]$,

$$
\left|\rho_{2}^{\prime}(t)-c_{\infty}+\lambda\right|+\left|\rho_{2}\left(t_{1}\right)-X_{0}\right| \leq 2 D_{0} \varepsilon^{1 / 2}
$$

Thus, to reach a contradiction, we only need to show (6-8).

Proof of Lemma 6.2. As in Lemmas A.1.4 and 5.2, the proof of (6-9)-(6-12) is based in an application of the implicit function theorem, and is very similar to the proof of [Martel and Merle 2008, Lemma A.1].

Equation (6-13) also follows from a simple computation, completely similar to (A-11) and (5-6).

Now we claim that from the definition of $T^{*}$ we can obtain an extra estimate on the parameter $\rho_{2}(t)$ :

$$
\rho_{2}(t) \geq \frac{1}{10}\left(c_{\infty}(\lambda)-\lambda\right) t_{1} \quad \text { for any } t \geq t_{1} .
$$

Indeed, from (6-7) and after integration between $t_{1}$ and $t \in\left[t_{1}, T^{*}\right]$ we have the bound

$$
\left|\rho_{2}(t)-\rho_{2}\left(t_{1}\right)-\left(c_{\infty}-\lambda\right)\left(t-t_{1}\right)\right| \leq \frac{1}{100}\left(t-t_{1}\right), \quad\left|\rho_{2}\left(t_{1}\right)-X_{0}\right| \leq \frac{1}{100} .
$$

Thus we have

$$
\left|\rho_{2}(t)-\left(c_{\infty}-\lambda\right) t\right| \leq \frac{1}{100}\left(t-t_{1}+1\right)+\left|\left(c_{\infty}-\lambda\right) t_{1}-X_{0}\right|
$$

In particular, for any $t \in\left[t_{1}, T^{*}\right]$ (recall that $\rho_{2}\left(t_{1}\right) \sim X_{0}>0$ )

$$
\rho_{2}(t) \geq\left(c_{\infty}-\lambda\right) t-\frac{1}{100}\left(t-t_{1}+1\right) \geq \frac{1}{10} c_{\infty} t
$$

This implies that the soliton is far away from the potential interaction region.

Now we prove (6-14) and (6-15). Set $y:=x-\rho_{2}(t)$. Taking the time derivative in the first orthogonality condition in (6-10) and using (6-13) we obtain

$$
\begin{aligned}
0=-c_{2}^{\prime}(t) \int_{\mathbb{R}} \Lambda Q_{c_{2}}\left(Q_{c_{2}}-z\right)+\left(c_{2}-\lambda-\rho_{2}^{\prime}\right)(t) \int_{\mathbb{R}} Q_{c_{2}}^{\prime} z-\frac{1}{2} \int_{\mathbb{R}} Q_{c_{2}}^{m}\left(\left(a_{\varepsilon}-2\right) z\right)_{x} \\
-\frac{\varepsilon}{2(m+1)} \int_{\mathbb{R}} a^{\prime}(\varepsilon x) Q_{c_{2}}^{m+1}(y)+\frac{1}{2} \int_{\mathbb{R}} Q_{c_{2}}^{\prime} a_{\varepsilon}\left((R+z)^{m}-R^{m}-m R^{m-1} z\right) .
\end{aligned}
$$

By scaling arguments,

$$
\int_{\mathbb{R}} \Lambda Q_{c_{2}} Q_{c_{2}}=\theta c_{2}^{2 \theta-1}(t) \int_{\mathbb{R}} Q^{2}
$$

Then, by redefining $\gamma$ if necessary,

$$
\left|\varepsilon \int_{\mathbb{R}} a^{\prime}(\varepsilon x) Q_{c_{2}}^{m+1}(y)\right| \leq K \varepsilon e^{-\gamma \varepsilon c_{2}(t) \rho_{2}(t)} \leq K \varepsilon e^{-\gamma \varepsilon t} .
$$

Similarly, from (6-16) and following (A-13) we have

$$
\left|\int_{\mathbb{R}} Q_{c_{2}}^{m}\left(\left(a_{\varepsilon}-2\right) z\right)_{x}\right| \leq K\|z(t)\|_{H^{1}(\mathbb{R})} e^{-\gamma \varepsilon t} .
$$


Finally, note that for $\gamma>0$ independent of $\varepsilon$,

$$
\left|\int_{\mathbb{R}} Q_{c_{2}}^{\prime} a_{\varepsilon}\left((R+z)^{m}-R^{m}-m R^{m-1} z\right)\right| \leq K \int_{\mathbb{R}} e^{-\gamma|y|} z^{2} .
$$

Collecting these estimates together, we have

$$
\frac{\left|c_{2}^{\prime}(t)\right|}{c_{2}(t)} \leq K \int_{\mathbb{R}} e^{-\gamma|y|} z^{2}+K\left|c_{2}(t)-\lambda-\rho_{2}^{\prime}(t)\right|\left(\int_{\mathbb{R}} e^{-\gamma|y|} z^{2}\right)^{\frac{1}{2}}+K e^{-\gamma \varepsilon t}\|z(t)\|_{H^{1}(\mathbb{R})}+K \varepsilon e^{-\gamma \varepsilon t} .
$$

On the other hand, by using the second orthogonality condition in (6-10), we have

$$
\begin{aligned}
0=\left(c_{2}-\lambda-\rho_{2}^{\prime}\right) & (t) \int_{\mathbb{R}} z(y R)_{x}+c_{2}^{\prime}(t) \int_{\mathbb{R}} y \Lambda Q_{c_{2}} z+\frac{1}{2}\left(c_{2}-\lambda-\rho_{2}^{\prime}\right)(t) \int_{\mathbb{R}} Q_{c_{2}}^{2} \\
+ & +\int_{\mathbb{R}}(y R)_{x}\left(\frac{1}{2} a_{\varepsilon}\left((R+z)^{m}-R^{m}-m R^{m-1} z\right)+\left(\frac{1}{2} a_{\varepsilon}(x)-1\right) Q_{c_{2}}^{m}\right) \\
& \quad+\int_{\mathbb{R}}(y R)_{x}\left(z_{x x}-c_{2} z+m R^{m-1} z\right)+\frac{m}{2} \int_{\mathbb{R}}(y R)_{x}\left(a_{\varepsilon}-2\right) R^{m-1} z .
\end{aligned}
$$

Note that by integration by parts,

$$
\int_{\mathbb{R}}(y R)_{x}\left(z_{x x}-c_{2} z+m R^{m-1} z\right)=\int_{\mathbb{R}} z\left(2 R+(m-3) R^{m}\right)=(m-3) \int_{\mathbb{R}} z R^{m} .
$$

Using the same arguments as in the precedent computations, we have

$$
\left|\left(c_{2}-\lambda-\rho_{2}^{\prime}\right)(t)\right| \leq K(m-3)\left(1+\frac{\left|c_{2}^{\prime}(t)\right|}{c_{2}(t)}\right)\left(\int_{\mathbb{R}} z^{2} e^{-\gamma|y|}\right)^{\frac{1}{2}}+K \int_{\mathbb{R}} z^{2} e^{-\gamma|y|}+\mid \int_{\mathbb{R}} Q_{c_{2}}^{m}(y)\left(a_{\varepsilon}-2\right) .
$$

From (6-16) and following (A-13) we have

$$
\left|\int_{\mathbb{R}} Q_{c_{2}}^{m}(y)\left(a_{\varepsilon}-2\right)\right| \leq K e^{-\gamma \varepsilon t} .
$$

Putting together (6-18) and the last estimates, we finally obtain the bounds in (6-11), and further we obtain (6-14) and (6-15), as desired.

Almost conserved quantities and monotonicity. We continue with a proof completely analogous to that of Proposition A.1.1. Recall from (2-8) the definition of the modified mass $\tilde{M}$.

Lemma 6.3 (Almost conservation of modified mass and energy). Consider $\tilde{M}=\tilde{M}[R]$ and $E_{a}=E_{a}[R]$, the modified mass and energy of the soliton $R$ of (6-9). For all $t \in\left[t_{1}, T^{*}\right]$ we have

$$
\begin{aligned}
& \tilde{M}[R](t)=\frac{1}{2} c_{2}^{2 \theta}(t) \int_{\mathbb{R}} Q^{2}+O\left(e^{-\varepsilon \gamma t}\right), \\
& E_{a}[R](t)=\frac{1}{2} c_{2}^{2 \theta}(t)\left(\lambda-\lambda_{0} c_{2}(t)\right) \int_{\mathbb{R}} Q^{2}+O\left(e^{-\varepsilon \gamma t}\right) .
\end{aligned}
$$

Furthermore, we have the bound

$$
\left|E_{a}[R]\left(t_{1}\right)-E_{a}[R](t)+\left(c_{2}\left(t_{1}\right)-\lambda\right)\left(\tilde{M}[R]\left(t_{1}\right)-\tilde{M}[R](t)\right)\right| \leq K\left|\left(\frac{c_{2}(t)}{c_{2}\left(t_{1}\right)}\right)^{2 \theta}-1\right|^{2}+K e^{-\varepsilon \gamma t_{1}} .
$$


Proof. We start by showing (6-19). We consider the case $m=2,4$, the case $m=3$ being easier. Note that from (2-8) that

$$
\tilde{M}[R](t)=\hat{M}[R](t)=\frac{1}{2} \int_{\mathbb{R}}\left(\frac{a_{\varepsilon}}{2}\right)^{1 / m} R^{2}=\frac{1}{2} c_{2}^{2 \theta}(t) \int_{\mathbb{R}} Q^{2}+\frac{1}{2} \int_{\mathbb{R}}\left(\left(\frac{a_{\varepsilon}(x)}{2}\right)^{1 / m}-1\right) R^{2} .
$$

From (6-16)-(6-17) and following the calculations in (A-13),

$$
\left|\int_{\mathbb{R}}\left(a_{\varepsilon}^{1 / m}(x)-2^{1 / m}\right) R^{2}\right| \leq K e^{-\gamma \varepsilon t}
$$

for some constants $K, \gamma>0$. Now we consider (6-20). Here we have

$$
\begin{aligned}
E_{a}[R](t) & =\frac{1}{2} \int_{\mathbb{R}} R_{x}^{2}+\frac{\lambda}{2} \int_{\mathbb{R}} R^{2}-\frac{1}{2(m+1)} \int_{\mathbb{R}} a_{\varepsilon} R^{m+1} \\
& =c_{2}^{2 \theta}(t)\left[c_{2}(t)\left(\frac{1}{2} \int_{\mathbb{R}} Q^{\prime 2}-\frac{1}{m+1} \int_{\mathbb{R}} Q^{m+1}\right)+\frac{\lambda}{2} \int_{\mathbb{R}} Q^{2}\right]+\frac{1}{m+1} \int_{\mathbb{R}}\left(1-\frac{1}{2} a_{\varepsilon}\right) R^{m+1} .
\end{aligned}
$$

Similarly to a recent computation, we have

$$
\left|\int_{\mathbb{R}}\left(2-a_{\varepsilon}(x)\right) R^{m+1}\right| \leq K e^{-\gamma \varepsilon t},
$$

for some constants $K, \gamma>0$. On the other hand, from Section A.6 we have

$$
\frac{1}{2} \int_{\mathbb{R}} Q^{\prime 2}-\frac{1}{m+1} \int_{\mathbb{R}} Q^{m+1}=-\frac{\lambda_{0}}{2} \int_{\mathbb{R}} Q^{2} \quad \text { and } \quad \lambda_{0}=\frac{5-m}{m+3},
$$

and thus

$$
E_{a}[R](t)=\frac{1}{2} c_{2}^{2 \theta}(t)\left(\lambda-\lambda_{0} c_{2}(t)\right) \int_{\mathbb{R}} Q^{2}+O\left(e^{-\gamma \varepsilon t}\right) .
$$

Combining both identities we have

$$
E_{a}[R](t)+\left(c_{2}\left(t_{1}\right)-\lambda\right) \hat{M}[R](t)=c_{2}^{2 \theta}(t)\left(c_{2}\left(t_{1}\right)-\lambda_{0} c_{2}(t)\right) M[Q]+O\left(e^{-\varepsilon \gamma t}\right) .
$$

In particular,

$$
\begin{aligned}
E_{a}[R]\left(t_{1}\right)-E_{a}[R](t)+ & \left(c_{2}\left(t_{1}\right)-\lambda\right)\left(\hat{M}[R]\left(t_{1}\right)-\hat{M}[R](t)\right) \\
= & \lambda_{0} M[Q]\left(c_{2}^{2 \theta+1}(t)-c_{2}^{2 \theta+1}\left(t_{1}\right)-\frac{c_{2}\left(t_{1}\right)}{\lambda_{0}}\left(c_{2}^{2 \theta}(t)-c_{2}^{2 \theta}\left(t_{1}\right)\right)\right)+O\left(e^{-\varepsilon \gamma t_{1}}\right) .
\end{aligned}
$$

To obtain the last estimate (6-21) we perform a Taylor development up to the second order (around $\left.y=y_{0}\right)$ of the function $g(y):=y^{\frac{2 \theta+1}{2 \theta}} ;$ and where $y:=c_{2}^{2 \theta}(t)$ and $y_{0}:=c_{2}^{2 \theta}\left(t_{1}\right)$. Note that $\frac{2 \theta+1}{2 \theta}=\frac{1}{\lambda_{0}}$ and $y_{0}^{1 / 2 \theta}=c_{2}\left(t_{1}\right)$. The conclusion follows at once.

To establish some stability properties for the function $u(t)$ we recall the mass $\tilde{M}[u]$ introduced in (2-8). We have that for $m=3$ and $0 \leq \lambda \leq \lambda_{0}$; and for $m=2,4$ and $0<\lambda \leq \lambda_{0}$,

$$
\tilde{M}[u](t)-\tilde{M}[u]\left(t_{1}\right) \leq 0 .
$$

for any $t \in\left[t_{1}, T^{*}\right]$. This result is a consequence of the remark on page 586 .

Now our objective is to estimate the quadratic term involved in (6-21). Following [Martel et al. 2002], we should use a "mass conservation" identity. However, since the mass is not conserved, estimate (6-22) 
is not enough to obtain a satisfactory estimate. Instead we will introduce a virial-type identity in the next lemma.

Let $\phi \in C(\mathbb{R})$ be an even function satisfying

$$
\begin{cases}\phi^{\prime} \leq 0 & \text { on }[0,+\infty) \\ \phi(x)=1 & \text { on }[0,1] \\ \phi(x)=e^{-x} & \text { on }[2,+\infty) \\ e^{-x} \leq \phi(x) \leq 3 e^{-x} & \text { on }[0,+\infty)\end{cases}
$$

Now, set $\psi(x):=\int_{0}^{x} \phi$. It is clear that $\psi$ an odd function. Moreover, for $|x| \geq 2$,

$$
\psi(+\infty)-\psi(|x|)=e^{-|x|} .
$$

Finally, for $A>0$, set

$$
\psi_{A}(x):=A(\psi(+\infty)+\psi(x / A))>0, \quad e^{-|x| / A} \leq \psi_{A}^{\prime}(x) \leq 3 e^{-|x| / A} .
$$

Note that $\lim _{x \rightarrow-\infty} \psi(x)=0$.

Lemma 6.4 (Virial-type estimate). There exist $K, A_{0}, \delta_{0}>0$ such that for all $t \in\left[t_{1}, T^{*}\right]$ and for some $\gamma=\gamma\left(c_{\infty}, A_{0}\right)>0$,

$$
\partial_{t} \int_{\mathbb{R}} z^{2}(t, x) \psi_{A_{0}}\left(x-\rho_{2}(t)\right) \leq-\delta_{0} \int_{\mathbb{R}}\left(z_{x}^{2}+z^{2}\right)(t, x) e^{-\frac{1}{A_{0}}\left|x-\rho_{2}(t)\right|}+K A_{0}\|z(t)\|_{H^{1}(\mathbb{R})} e^{-\gamma \varepsilon t} .
$$

For the proof, see Section A.4.

We can improve the estimate (6-21):

Corollary 6.5 (Quadratic control of the variation of $c_{2}(t)$ ).

$$
\begin{aligned}
&\left|E_{a}[R]\left(t_{1}\right)-E_{a}[R](t)+\left(c_{2}\left(t_{1}\right)-\lambda\right)\left(\tilde{M}[R]\left(t_{1}\right)-\tilde{M}[R](t)\right)\right| \\
& \leq K\|z(t)\|_{H^{1}(\mathbb{R})}^{4}+K\left\|z\left(t_{1}\right)\right\|_{H^{1}(\mathbb{R})}^{4}+K e^{-\varepsilon \gamma t_{1}} .
\end{aligned}
$$

Proof. From (6-15) and taking $A_{0}$ large enough (but fixed and independent of $\varepsilon$ ) in Lemma 6.4, we have after an integration of (6-26) that

$$
\left|c_{2}(t)-c_{2}\left(t_{1}\right)\right| \leq K A_{0}\|z(t)\|_{L^{2}(\mathbb{R})}^{2}+K A_{0}\left\|z\left(t_{1}\right)\right\|_{L^{2}(\mathbb{R})}^{2}+K A_{0} D_{0} \varepsilon^{-1 / 2} e^{-\gamma \varepsilon t_{1}} .
$$

Substituting this in (6-21) and taking $\gamma$ even smaller, we get the conclusion.

Energy estimates. Let us now introduce the second-order functional

$\mathscr{F}_{2}(t):=\frac{1}{2} \int_{\mathbb{R}}\left(z_{x}^{2}+\left(\lambda+\left(c_{2}\left(t_{1}\right)-\lambda\right)\left(\frac{a_{\varepsilon}}{2}\right)^{1 / m}\right) z^{2}\right)-\frac{1}{2(m+1)} \int_{\mathbb{R}} a_{\varepsilon}\left((R+z)^{m+1}-R^{m+1}-(m+1) R^{m} z\right)$.

This functional, related to the Weinstein functional, have the following properties.

Lemma 6.6 (Energy expansion). Consider the energy $E_{a}[u]$ and the mass $\tilde{M}[u]$ defined in (1-21) and (2-8). For all $t \in\left[t_{1}, T^{*}\right]$,

$$
E_{a}[u](t)+\left(c_{2}\left(t_{1}\right)-\lambda\right) \tilde{M}[u](t)=E_{a}[R]+\left(c_{2}\left(t_{1}\right)-\lambda\right) \tilde{M}[R]+\mathscr{F}_{2}(t)+O\left(e^{-\gamma \varepsilon t}\|z(t)\|_{H^{1}(\mathbb{R})}\right) .
$$


Proof. Using the orthogonality condition (6-10), we have

$E_{a}[u](t)=E_{a}[R]-\int_{\mathbb{R}} z\left(a_{\varepsilon}-2\right) R^{m}+\frac{1}{2} \int_{\mathbb{R}} z_{x}^{2}+\frac{\lambda}{2} \int_{\mathbb{R}} z^{2}-\frac{1}{m+1} \int_{\mathbb{R}} a_{\varepsilon}\left((R+z)^{m+1}-R^{m+1}-(m+1) R^{m} z\right)$.

Moreover, following (A-13), we easily get

$$
\left|\int_{\mathbb{R}} z\left(a_{\varepsilon}-2\right) R^{m}\right| \leq K e^{-\gamma \varepsilon t}\|z(t)\|_{H^{1}(\mathbb{R})} .
$$

Similarly,

$$
\hat{M}[u](t)=\hat{M}[R]+\hat{M}[z]+\int_{\mathbb{R}}\left(\left(\frac{a_{\varepsilon}}{2}\right)^{1 / m}-1\right) R z=\hat{M}[R]+\hat{M}[z]+O\left(e^{-\varepsilon \gamma t}\|z(t)\|_{H^{1}(\mathbb{R})}\right) .
$$

Combining these estimates, we have

$$
\begin{array}{r}
E_{a}[u](t)+\left(c_{2}\left(t_{1}\right)-\lambda\right) \tilde{M}[u](t)=E_{a}[R]+\left(c_{2}\left(t_{1}\right)-\lambda\right) \tilde{M}[R]+\frac{1}{2} \int_{\mathbb{R}}\left(z_{x}^{2}+\left(\left(c_{2}\left(t_{1}\right)-\lambda\right)\left(\frac{a_{\varepsilon}}{2}\right)^{1 / m}+\lambda\right) z^{2}\right) \\
-\frac{1}{2(m+1)} \int_{\mathbb{R}} a_{\varepsilon}\left((R+z)^{m+1}-R^{m+1}-(m+1) R^{m} z\right)+O\left(e^{-\gamma \varepsilon t}\|z(t)\|_{H^{1}(\mathbb{R})}\right) .
\end{array}
$$

This concludes the proof.

Lemma 6.7 (Modified coercivity for $\mathscr{F}_{2}$ ). There exists $\varepsilon_{0}>0$ such that for all $0<\varepsilon<\varepsilon_{0}$ the following hold. There exist $K, \tilde{\lambda}_{0}>0$, independent of $K^{*}$ such that for every $t \in\left[t_{1}, T^{*}\right]$

$$
\mathscr{F}_{2}(t) \geq \tilde{\lambda}_{0}\|z(t)\|_{H^{1}(\mathbb{R})}^{2}-K \varepsilon e^{-\gamma \varepsilon t}\|z(t)\|_{L^{2}(\mathbb{R})}^{2}+O\left(\|z(t)\|_{L^{2}(\mathbb{R})}^{3}\right) .
$$

Proof. Note that

$$
\begin{aligned}
\mathscr{F}_{2}(t)=\frac{1}{2} \int_{\mathbb{R}}\left(z_{x}^{2}+\left(\left(c_{2}\left(t_{1}\right)-\lambda\right)\left(\frac{a_{\varepsilon}}{2}\right)^{1 / m}\right.\right. & \left.+\lambda) z^{2}\right) \\
& -\frac{m}{2} \int_{\mathbb{R}} Q_{c_{2}}^{m-1} z^{2}+O\left(\|z(t)\|_{H^{1}(\mathbb{R})}^{3}\right)+O\left(e^{-\gamma \varepsilon t}\|z(t)\|_{H^{1}(\mathbb{R})}^{2}\right) .
\end{aligned}
$$

Now take $R_{0}>0$ independent of $\varepsilon$, to be fixed later. Consider the function

$$
\phi_{R_{0}}(t, x):=\phi\left(\frac{x-\rho_{2}(t)}{R_{0}}\right)
$$

where $\phi$ is defined in (6-23). We split the analysis according to the decomposition $1=\phi_{R_{0}}+\left(1-\phi_{R_{0}}\right)$. Inside the region $\left|x-\rho_{2}(t)\right| \leq R_{0}$, we have, a consequence of (1-13),

$$
2-a_{\varepsilon}(x) \leq K e^{-\gamma \varepsilon|x|} \leq K e^{\gamma \varepsilon R_{0}} e^{-\gamma \varepsilon \rho_{2}(t)} .
$$

Outside this region, we have $\phi_{R_{0}} \geq e^{-R_{0}}$. Thus

$$
\int_{\mathbb{R}} \phi_{R_{0}}\left(\left(c_{2}\left(t_{1}\right)-\lambda\right)\left(\frac{a_{\varepsilon}}{2}\right)^{1 / m}+\lambda\right) z^{2} \geq\left(c_{2}\left(t_{1}\right)-K e^{\gamma \varepsilon R_{0}} e^{-\gamma \varepsilon \rho_{2}(t)}\right) \int_{\mathbb{R}} \phi_{R_{0}} z^{2},
$$

for fixed $K, \gamma>0$. 
On the other hand, $\left|\left(1-\phi_{R_{0}}\right) Q_{c_{2}}\right| \leq K e^{-\gamma R_{0}}$, and thus

$$
\begin{aligned}
\int_{\mathbb{R}}\left(1-\phi_{R_{0}}\right)\left(\left(c_{2}\left(t_{1}\right)-\lambda\right)\left(\frac{a_{\varepsilon}}{2}\right)^{1 / m}+\lambda\right) z^{2} & -\frac{m}{2} \int_{\mathbb{R}}\left(1-\phi_{R_{0}}\right) Q_{c_{2}}^{m-1} z^{2} \\
& \geq\left(\left(c_{2}\left(t_{1}\right)-\lambda\right)\left(\frac{1}{2}\right)^{1 / m}+\lambda-K e^{-\gamma R_{0}}\right) \int_{\mathbb{R}}\left(1-\phi_{R_{0}}\right) z^{2},
\end{aligned}
$$

for fixed $K, \gamma>0$. Taking $R_{0}=R_{0}(m, \lambda)$ large enough, we have

$$
(6-29) \geq \frac{1}{2^{1 / m}} c_{2}\left(t_{1}\right) \int_{\mathbb{R}}\left(1-\phi_{R_{0}}\right) z^{2} .
$$

Therefore,

$$
\begin{aligned}
\mathscr{F}_{2}(t) \geq & \frac{1}{2} \int_{\mathbb{R}} \phi_{R_{0}}\left(z_{x}^{2}+c_{2}\left(t_{1}\right) z^{2}-m Q_{c_{2}}^{m-1} z^{2}\right)+\frac{1}{2} \int_{\mathbb{R}}\left(1-\phi_{R_{0}}\right)\left(z_{x}^{2}+\left(\frac{1}{2}\right)^{1 / m} c_{2}\left(t_{1}\right) z^{2}\right) \\
& -K e^{\gamma \varepsilon R_{0}} e^{-\gamma \varepsilon \rho_{2}(t)} \int_{\mathbb{R}} \phi_{R_{0}} z^{2}+O\left(\|z(t)\|_{H^{1}(\mathbb{R})}^{3}\right)+O\left(e^{-\gamma \varepsilon t}\|z(t)\|_{H^{1}(\mathbb{R})}^{2}\right) .
\end{aligned}
$$

Taking $R_{0}$ even larger if necessary (but independent of $\varepsilon$ ), and using a localization argument as in [Martel and Merle 2002b], we conclude that there exists $\tilde{\lambda}_{0}>0$ such that

$$
\mathscr{F}_{2}(t) \geq \tilde{\lambda}_{0} \int_{\mathbb{R}}\left(z_{x}^{2}+z^{2}\right)-K e^{\gamma \varepsilon R_{0}} e^{-\gamma \varepsilon \rho_{2}(t)} \int_{\mathbb{R}} \phi_{R_{0}} z^{2}+O\left(\|z(t)\|_{H^{1}(\mathbb{R})}^{3}\right)+O\left(e^{-\gamma \varepsilon t}\|z(t)\|_{H^{1}(\mathbb{R})}^{2}\right) .
$$

Finally, taking $\varepsilon_{0}$ smaller if necessary, we have

$$
\mathscr{F}_{2}(t) \geq \tilde{\lambda}_{0} \int_{\mathbb{R}}\left(z_{x}^{2}+z^{2}\right)+O\left(\|z(t)\|_{H^{1}(\mathbb{R})}^{3}\right)+O\left(e^{-\gamma \varepsilon t}\|z(t)\|_{H^{1}(\mathbb{R})}^{2}\right),
$$

for a new constant $\tilde{\lambda}_{0}>0$.

Conclusion of the proof of Theorem 6.1(1). Now we prove that our assumption $T^{*}<+\infty$ must lead to a contradiction. Indeed, from Lemmas 6.6 and 6.7, we have for all $t \in\left[t_{1}, T^{*}\right]$ and for some constant $K>0$,

$$
\begin{aligned}
\|z(t)\|_{H^{1}(\mathbb{R})}^{2} \leq & K \mathscr{F}_{2}\left(t_{1}\right)+E_{a}[u](t)-E_{a}[u]\left(t_{1}\right)+\left(c_{2}\left(t_{1}\right)-\lambda\right)\left[\tilde{M}[u](t)-\tilde{M}[u]\left(t_{1}\right)\right] \\
& +E_{a}[R]\left(t_{1}\right)-E_{a}[R](t)+\left(c_{2}\left(t_{1}\right)-\lambda\right)\left[\tilde{M}[R]\left(t_{1}\right)-\tilde{M}[R](t)\right] \\
& +K \varepsilon \sup _{t \in\left[t_{1}, T^{*}\right]} e^{-\gamma \varepsilon t}\|z(t)\|_{L^{2}(\mathbb{R})}+K \sup _{t \in\left[t_{1}, T^{*}\right]}\|z(t)\|_{L^{2}(\mathbb{R})}^{3} .
\end{aligned}
$$

From Lemmas 6.2 and 6.3, Corollary 6.5 and the conservation we have

$$
\begin{aligned}
\|z(t)\|_{H^{1}(\mathbb{R})}^{2} \leq K \varepsilon+\left(c_{2}\left(t_{1}\right)-\lambda\right)\left(\tilde{M}[u](t)-\tilde{M}[u]\left(t_{1}\right)\right) & \\
& +K \sup _{t \in\left[t_{1}, T^{*}\right]}\|z(t)\|_{H^{1}(\mathbb{R})}^{4}+K e^{-\varepsilon \gamma t_{1}}\left(1+D_{0} \varepsilon^{1 / 2}\right)+K D_{0}^{3} \varepsilon^{3 / 2} .
\end{aligned}
$$

Finally, from (6-22) we have $\tilde{M}[u](t)-\tilde{M}[u]\left(t_{1}\right) \leq 0$. Collecting the preceding estimates we have for $\varepsilon>0$ small and $D_{0}=D_{0}(K)$ large enough

$$
\|z(t)\|_{H^{1}(\mathbb{R})}^{2} \leq \frac{1}{4} D_{0}^{2} \varepsilon
$$


which contradicts the definition of $T^{*}$. The conclusion is that

$$
\sup _{t \geq t_{1}}\left\|u(t)-2^{-1 /(m-1)} Q_{c_{2}(t)}\left(\cdot-\rho_{2}(t)\right)\right\|_{H^{1}(\mathbb{R})} \leq K \varepsilon^{1 / 2} .
$$

Using (6-11), we finally get (6-2).

Proof of Theorem 6.1(2): asymptotic stability. Now we prove (6-3) in Theorem 6.1, following closely [Martel and Merle 2005; 2008].

We continue with the notation introduced in the proof of the stability property (6-2). We have to show the existence of $K, c^{+}>0$ such that

$$
\lim _{t \rightarrow+\infty}\left\|u(t)-Q_{c^{+}}\left(\cdot-\rho_{2}(t)\right)\right\|_{H^{1}\left(x>\frac{1}{10} c_{\infty} t\right)}=0 \quad \text { and } \quad\left|c_{\infty}-c^{+}\right| \leq K \varepsilon^{1 / 2} .
$$

From the stability result above stated it is easy to check that the decomposition proved in Lemma 6.2 and all its conclusions hold for any time $t \geq t_{1}$.

Monotony for mass and energy. The next step is to prove some monotonicity formulae for local mass and energy. Let $K_{0}>0$ and set

$$
\phi(x):=\frac{2}{\pi} \arctan \left(e^{x / K_{0}}\right) .
$$

It is clear that $\lim _{x \rightarrow+\infty} \phi(x)=1$ and $\lim _{x \rightarrow-\infty} \phi(x)=0$. In addition, $\phi(-x)=1-\phi(x)$ for all $x \in \mathbb{R}$, and

$$
0<\phi^{\prime}(x)=\frac{2}{\pi K_{0}} \frac{e^{x / K}}{1+e^{2 x / K_{0}}} ; \quad \phi^{(3)}(x) \leq \frac{1}{K_{0}^{2}} \phi^{\prime}(x) .
$$

Moreover, we have $1-\phi(x) \leq K e^{-x / K_{0}}$ as $x \rightarrow+\infty$, and $\phi(x) \leq K e^{x / K_{0}}$ as $x \rightarrow-\infty$.

Let $\sigma, x_{0}>0$. We define, for $t, t_{0} \geq t_{1}$, and $\tilde{y}\left(x_{0}\right):=x-\left(\rho_{2}\left(t_{0}\right)+\sigma\left(t-t_{0}\right)+x_{0}\right)$,

$$
I_{x_{0}, t_{0}}(t):=\int_{\mathbb{R}} u^{2}(t, x) \phi\left(\tilde{y}\left(x_{0}\right)\right) d x, \quad \tilde{I}_{x_{0}, t_{0}}(t):=\int_{\mathbb{R}} u^{2}(t, x) \phi\left(\tilde{y}\left(-x_{0}\right)\right) d x,
$$

and

$$
J_{x_{0}, t_{0}}:=\int_{\mathbb{R}}\left(u_{x}^{2}+u^{2}-\frac{2 a_{\varepsilon}}{m+1} u^{m+1}\right)(t, x) \phi\left(\tilde{y}\left(x_{0}\right)\right) d x .
$$

Lemma 6.8 (Monotony formulae). Suppose $0<\sigma<\frac{1}{2}\left(c_{\infty}(\lambda)-\lambda\right)$ and $K_{0}>\sqrt{\frac{2}{\sigma}}$. There exists $K, \varepsilon_{0}>0$ small enough such that for all $0<\varepsilon<\varepsilon_{0}$ and for all $t, t_{0} \geq t_{1}$ with $t_{0} \geq t$ we have

$$
I_{x_{0}, t_{0}}\left(t_{0}\right)-I_{x_{0}, t_{0}}(t) \leq K\left(e^{-x_{0} / K_{0}}+\varepsilon^{-1} e^{-\gamma \varepsilon T_{\varepsilon}} e^{-\varepsilon \gamma x_{0} / K_{0}}\right) .
$$

On the other hand, if $t \geq t_{0}$ and $\rho_{2}\left(t_{0}\right) \geq t_{1}+x_{0}$,

$$
\tilde{I}_{x_{0}, t_{0}}(t)-\tilde{I}_{x_{0}, t_{0}}\left(t_{0}\right) \leq K\left(e^{-x_{0} / K_{0}}+\varepsilon^{-1} e^{-\varepsilon \gamma \rho_{2}\left(t_{0}\right)} e^{\varepsilon \gamma x_{0} / K_{0}}\right),
$$

and finally if $t_{0} \geq t$,

$$
J_{x_{0}, t_{0}}\left(t_{0}\right)-J_{x_{0}, t_{0}}(t) \leq K\left(e^{-x_{0} / K_{0}}+\varepsilon^{-1} e^{-\gamma \varepsilon T_{\varepsilon}} e^{-\varepsilon \gamma x_{0} / K_{0}}\right) .
$$

The proof is given in Section A.5. 
Conclusion of the proof of Theorem 6.1(2). Consider $0<\varepsilon<\varepsilon_{0}$ and $u(t)$ satisfying (6-1). From Lemma 6.2, we can decompose $u(t)$ for all $t \geq t_{1}$ such that $u(t, x)=2^{-1 /(m-1)} Q_{c_{2}(t)}\left(x-\rho_{2}(t)\right)+z(t, x)$, where $z$ satisfies (6-10), (6-11), (6-12), (6-14) and (6-15). An application of Lemma 6.4 followed by integration in time shows that there exists $K=K\left(D_{0}\right)>0$ such that

$$
\int_{t_{1}}^{+\infty} \int_{\mathbb{R}}\left(z_{x}^{2}+z\right)(t, x) e^{-\frac{1}{A_{0}}\left|x-\rho_{2}(t)\right|} \leq K\left(D_{0}\right) \varepsilon
$$

Now we claim that

$$
c^{+}:=\lim _{t \rightarrow+\infty} c_{2}(t)<+\infty \quad \text { and } \quad\left|c^{+}-c_{\infty}\right| \leq K \varepsilon^{1 / 2} .
$$

In fact, note that from (6-35) there exists a sequence $t_{n} \uparrow+\infty, t_{n} \in[n, n+1)$ such that

$$
\lim _{n \rightarrow+\infty} \int_{\mathbb{R}}\left(z_{x}^{2}+z\right)\left(t_{n}, x\right) e^{-\frac{1}{A_{0}}\left|x-\rho_{2}\left(t_{n}\right)\right|}=0 .
$$

From (6-37), (6-14), and (6-15), and taking $A_{0}>0$ so large that $1 / A_{0}<\gamma$, we get

$$
\left|c_{2}^{\prime}(t)\right| \leq K \int_{\mathbb{R}} z^{2}(t, x) e^{-\frac{1}{A_{0}}\left|x-\rho_{2}(t)\right|}+K e^{-\gamma \varepsilon t} .
$$

This, combined with (6-35) and (6-12), allows us to conclude (6-36). This proves the first part of (6-4).

The next step is to prove that

$$
\limsup _{t \rightarrow+\infty} \int_{\mathbb{R}}\left(z_{x}^{2}+z^{2}\right)\left(t, x+\rho_{2}(t)\right) \phi\left(x-x_{0}\right) \leq K e^{-x_{0} / 2 K_{0}}+K \varepsilon^{-1} e^{-\varepsilon \gamma T_{\varepsilon}} e^{-\varepsilon \gamma x_{0} / K_{0}} .
$$

This follows from the decay properties of $R$ and the estimate

$$
\limsup _{t \rightarrow+\infty} \int_{\mathbb{R}}\left(u_{x}^{2}+u^{2}\right)\left(t, x+\rho_{2}(t)\right) \phi\left(x-x_{0}\right) \leq K e^{-x_{0} / 2 K_{0}}+K \varepsilon^{-1} e^{-\varepsilon \gamma T_{\varepsilon}} e^{-\varepsilon \gamma x_{0} / K_{0}},
$$

which we prove now. We start from (6-34): we have for $t_{0} \geq t_{1}$,

$$
J_{x_{0}, t_{0}}\left(t_{0}\right) \leq J_{x_{0}, t_{0}}\left(t_{1}\right)+K e^{-x_{0} / K_{0}}+K \varepsilon^{-1} e^{-\varepsilon \gamma T_{\varepsilon}} e^{-\varepsilon \gamma x_{0} / K_{0}} .
$$

From the equivalence between the energy and $H^{1}$-norm (we are in a subcritical case), we have

$$
\begin{aligned}
\int_{\mathbb{R}}\left(u_{x}^{2}+u^{2}\right)\left(t_{0}, x+\rho_{2}\left(t_{0}\right)\right) \phi\left(x-x_{0}\right) \leq & K \int_{\mathbb{R}}\left(u_{x}^{2}+u^{2}\right)\left(t_{1}, x+\rho_{2}\left(t_{1}\right)\right) \phi\left(x-y_{0}\right) \\
& +K e^{-x_{0} / 2 K}+K \varepsilon^{-1} e^{-\varepsilon \gamma T_{\varepsilon}} e^{-\varepsilon \gamma x_{0} / K_{0}},
\end{aligned}
$$

where $y_{0}:=\rho_{2}\left(t_{0}\right)-\rho_{2}\left(t_{1}\right)+\sigma\left(t_{1}-t_{0}\right)+x_{0}$. Now we send $t_{0} \rightarrow+\infty$ noticing that $y_{0} \rightarrow+\infty$. This gives (6-38), as desired.

We next prove that

$$
\lim _{n \rightarrow+\infty} \int_{\mathbb{R}}\left(z_{x}^{2}+z^{2}\right)\left(t_{n}, x\right) \phi\left(x-\rho_{2}\left(t_{n}\right)+x_{0}\right) d x=0 .
$$


where $\left(t_{n}\right)_{n \in \mathbb{N}}$ is the sequence from (6-39). Indeed, for any $x_{1}>0$,

$$
\begin{aligned}
\int_{\mathbb{R}}\left(z_{x}^{2}\right. & \left.+z^{2}\right)\left(t_{n}, x+\rho_{2}\left(t_{n}\right)\right) \phi\left(x+x_{0}\right) \\
& \leq K\left(e^{\frac{x_{0}}{A_{0}}}+e^{\frac{x_{1}}{A_{0}}}\right) \int_{\mathbb{R}}\left(z_{x}^{2}+z^{2}\right)\left(t_{n}, x+\rho_{2}\left(t_{n}\right)\right) e^{-\frac{|x|}{A_{0}}}+K \int_{\mathbb{R}}\left(z_{x}^{2}+z^{2}\right)\left(t_{n}, x+\rho_{2}\left(t_{n}\right)\right) \phi\left(x-x_{1}\right) .
\end{aligned}
$$

Using (6-39) we are able to take the limit $n \rightarrow+\infty$ in this inequality, with $x_{0}, x_{1}$ fixed. Taking the limit $x_{1} \rightarrow+\infty$ yields the conclusion.

We finally prove that the above result holds for any sequence $t_{n} \rightarrow+\infty$. Let $\beta<c_{\infty}(\lambda)-\lambda$ to be fixed. We want to prove that for $\varepsilon$ small enough,

$$
\lim _{t \rightarrow+\infty} \int_{\mathbb{R}}\left(z_{x}^{2}+z^{2}\right)(t, x) \phi(x-\beta t) d x=0 .
$$

First, we claim that for any $t_{2}, t_{3}>t_{1}$ with $t_{2}<t_{3}$ and $\rho_{2}\left(t_{2}\right)>x_{0}+t_{1}$, we have

$$
\int_{\mathbb{R}} u^{2}\left(t_{3}, x\right) \phi\left(x-y_{3}\right) d x \leq \int_{\mathbb{R}} u^{2}\left(t_{2}, x\right) \phi\left(x-y_{2}\right) d x+K e^{-x_{0} / K_{0}}+K \varepsilon^{-1} e^{-\gamma \varepsilon \rho_{2}\left(t_{2}\right)} e^{\gamma \varepsilon x_{0} / K_{0}},
$$

where $y_{3}:=\rho_{2}\left(t_{2}\right)+\frac{1}{2} \beta\left(t_{3}-t_{2}\right)-x_{0}$ and $y_{2}:=\rho_{2}\left(t_{2}\right)-x_{0}$. In fact, the left-hand side of this inequality corresponds to $\tilde{I}_{x_{0}, t_{2}}\left(t_{3}\right)$ and the right one is $\tilde{I}_{x_{0}, t_{2}}\left(t_{2}\right)$, with $\sigma:=\frac{1}{2} \beta$ (see (6-31) for the definitions). Thus (6-40) a consequence of Lemma 6.8, more specifically of (6-33).

Now the rest of the proof is similar to [Martel and Merle 2005]. Since $\int_{\mathbb{R}} z\left(t, x+\rho_{2}(t)\right) R(x)=0$, we have

$$
\left|\int_{\mathbb{R}} z\left(t, x+\rho_{2}(t)\right) R(x) \phi\left(x+x_{0}\right)\right| \leq K \varepsilon^{1 / 2} e^{-x_{0} / 2 K_{0}} .
$$

Second, we use the decomposition $u(t, x)=2^{-1 /(m-1)} Q_{c_{2}(t)}\left(x-\rho_{2}(t)\right)+z(t, x)$ in (6-40) to get

$$
\begin{aligned}
& \int_{\mathbb{R}} z^{2}\left(t_{3}, x\right) \phi\left(x-y_{3}\right) d x \\
& \quad \leq \int_{\mathbb{R}} z^{2}\left(t_{2}, x\right) \phi\left(x-y_{2}\right) d x+K e^{-x_{0} / 2 K_{0}}+K \varepsilon^{-1} e^{-\gamma \varepsilon \rho_{2}\left(t_{2}\right)} e^{\gamma \varepsilon x_{0} / K_{0}}+K\left|c_{2}\left(t_{2}\right)-c_{2}\left(t_{3}\right)\right| .
\end{aligned}
$$

Third, consider $t>t_{1}$ large, and define $t^{\prime} \in\left(t_{1}, t\right)$ such that $\beta t:=\rho_{2}\left(t^{\prime}\right)+\frac{\beta}{2}\left(t-t^{\prime}\right)-x_{0}$. Note that $t^{\prime} \rightarrow+\infty$ as $t \rightarrow+\infty$. Since $t_{n} \in[n, n+1)$ there exists $n=n(t)$ such that $0<t-t_{n} \leq 2$, and then

$$
\beta t:=\rho_{2}\left(t_{n}\right)+\frac{1}{2} \beta\left(t-t_{n}\right)-\tilde{x}_{0}, \quad \text { with }\left|\tilde{x}_{0}-x_{0}\right| \leq 10 .
$$

Now we apply (6-41) between $t_{3}=t$ and $t_{2}=t_{n}$. We get

$$
\begin{aligned}
& \int_{\mathbb{R}} z^{2}(t, x) \phi(x-\beta t) d x \\
& \quad \leq \int_{\mathbb{R}} z^{2}\left(t_{n}, x\right) \phi\left(x-\rho_{2}\left(t_{n}\right)+\tilde{x}_{0}\right) d x+K e^{-x_{0} / 2 K_{0}}+K \varepsilon^{-1} e^{-\gamma \varepsilon \rho_{2}\left(t_{n}\right)} e^{\gamma \varepsilon x_{0} / K_{0}}+K\left|c_{2}(t)-c_{2}\left(t_{n}\right)\right| .
\end{aligned}
$$

Since $n(t) \rightarrow+\infty$ as $t \rightarrow+\infty$, by (6-39) and (6-36) we obtain

$$
\limsup _{t \rightarrow+\infty} \int_{\mathbb{R}} z^{2}(t, x) \phi(x-\beta t) d x \leq K e^{-x_{0} / 2 K_{0}},
$$


and since $x_{0}$ is arbitrary (because of $\lim _{t \rightarrow+\infty} \rho_{2}\left(t_{n}\right)=+\infty$ ), we get the desired result. The same result is still valid for $z_{x}$. We have

$$
\limsup _{t \rightarrow+\infty} \int_{\mathbb{R}} z_{x}^{2}(t, x) \phi(x-\beta t) d x \leq K e^{-x_{0} / 2 K_{0}} .
$$

Finally, let

$$
\begin{aligned}
w^{+}(t, x): & =u(t, x)-2^{-1 /(m-1)} Q_{c^{+}}\left(x-\rho_{2}(t)\right) \\
& =z(t, x)+2^{-1 /(m-1)}\left(Q_{c_{2}(t)}\left(x-\rho_{2}(t)\right)-Q_{c^{+}}\left(x-\rho_{2}(t)\right)\right) .
\end{aligned}
$$

From this and (6-36) we obtain (6-3).

\section{Proof of the main theorems}

Proof of Theorem 1.1. We will combine Theorems 3.1 and 4.1 to obtain the global solution $u(t)$ with the required properties. This method was employed earlier in [Martel and Merle 2007; Martel et al. 2010; Muñoz 2010].

By Theorem 3.1 there exists a solution $u$ of (1-15) satisfying $u \in C\left(\mathbb{R}, H^{1}(\mathbb{R})\right)$ and (3-1). This solution also satisfies, from (3-3),

$$
\left\|u\left(-T_{\varepsilon}\right)-Q\left(\cdot+(1-\lambda) T_{\varepsilon}\right)\right\|_{H^{1}(\mathbb{R})} \leq K \varepsilon^{10},
$$

for $\varepsilon$ small enough. In addition, $u$ is unique if $m=2,4$ and $\lambda>0$, or if $m=3$ and $\lambda \geq 0$. This proves Theorem 1.1(1).

To obtain Theorem 1.1(2) we invoke (4-2) and (4-3) in Theorem 4.1. We define $\tilde{T}_{\varepsilon}:=T_{\varepsilon}+\rho_{1}\left(T_{\varepsilon}\right)$, and $\rho_{\varepsilon}:=\rho\left(T_{\varepsilon}\right)$. Now (1-27) and (1-28) are straightforward.

Proof of Theorem 1.2. Suppose $m=2,3,4$ with $\lambda>0$ for $m=2$, 4. Define $t_{1}:=T_{\varepsilon}+\rho_{1}\left(T_{\varepsilon}\right)$ and $X_{0}:=\rho\left(T_{\varepsilon}\right)$. Then, from the preceding estimates and Theorem 6.1 we have stability and asymptotic stability at infinity. In other words, there exist a constant $c^{+}>0$ and a $C^{1}$ function $\rho_{2}(t) \in \mathbb{R}$ such that

$$
w^{+}(t):=u(t)-2^{-1 /(m-1)} Q_{c^{+}}\left(\cdot-\rho_{2}(t)\right)
$$

satisfies (6-2) and (6-3). This proves (1-29) and (1-30).

We finally prove (1-32) and (1-33). From the energy conservation, we have for all $t \geq t_{1}$,

$$
E_{a}[u](-\infty)=E_{a}\left[2^{-1 /(m-1)} Q_{c^{+}}\left(\cdot-\rho_{2}(t)\right)+w^{+}(t)\right]
$$

In particular, from (6-3) and Section A.6 we have as $t \rightarrow+\infty$

$$
\left(\lambda-\lambda_{0}\right) M[Q]=\frac{\left(c^{+}\right)^{2 \theta}}{2^{2 /(m-1)}}\left(\lambda-\lambda_{0} c^{+}\right) M[Q]+E^{+} .
$$


From this identity $E^{+}:=\lim _{t \rightarrow+\infty} E_{a}\left[w^{+}\right](t)$ is well defined. This proves (1-32). To deal with (1-33), note that from the stability result (6-2) and the Morrey embedding we have, for any $\lambda>0$,

$$
\begin{aligned}
E\left[w^{+}\right](t) & =\frac{1}{2} \int_{\mathbb{R}}\left(w_{x}^{+}\right)^{2}(t)+\frac{\lambda}{2} \int_{\mathbb{R}}\left(w^{+}\right)^{2}(t)-\frac{1}{m+1} \int_{\mathbb{R}} a_{\varepsilon}\left(w^{+}\right)^{m+1}(t) \\
& \geq \frac{1}{2} \int_{\mathbb{R}}\left(w_{x}^{+}\right)^{2}(t)+\frac{\lambda}{2} \int_{\mathbb{R}}\left(w^{+}\right)^{2}(t)-K \varepsilon^{(m-1) / 2} \int_{\mathbb{R}} a_{\varepsilon}\left(w^{+}\right)^{2}(t) \geq \mu\left\|w^{+}(t)\right\|_{H^{1}(\mathbb{R})}^{2}
\end{aligned}
$$

for some $\mu=\mu(\lambda)>0$. Passing to the limit we obtain (1-33).

Now we prove the bound (1-34). First, the treat the cubic case with $\lambda=0$. Here, from (7-1) we have

$$
E^{+}=\lambda_{0}\left(\frac{\left(c^{+}\right)^{3 / 2}}{2^{2 /(m-1)}}-1\right) M[Q]
$$

Since in this case we have $2^{2 /(m-1)}=2=c_{\infty}^{3 / 2}, M[Q]=2$ and $\lambda_{0}=\frac{1}{3}$, we obtain $\frac{3}{2} E^{+}=\left(\frac{c^{+}}{c_{\infty}}\right)^{3 / 2}-1$.

Now we deal with the case $\lambda>0$. After some algebraic manipulations, the equation for $c_{\infty}$ in (4-17) becomes

$$
\frac{c_{\infty}^{2 \theta}}{2^{2 /(m-1)}}\left(\lambda_{0} c_{\infty}-\lambda\right) M[Q]=\left(\lambda_{0}-\lambda\right) M[Q]
$$

On the other hand, from (7-1) and (1-33) we have

$$
\mu \limsup _{t \rightarrow+\infty}\left\|w^{+}(t)\right\|_{H^{1}(\mathbb{R})}^{2} \leq \frac{\left(c^{+}\right)^{2 \theta}}{2^{2 /(m-1)}}\left(\lambda_{0} c^{+}-\lambda\right) M[Q]-\left(\lambda_{0}-\lambda\right) M[Q] .
$$

Putting together both estimates, we get

$$
\tilde{\mu} \limsup _{t \rightarrow+\infty}\left\|w^{+}(t)\right\|_{H^{1}(\mathbb{R})}^{2} \leq\left(c^{+}\right)^{2 \theta+1}-c_{\infty}^{2 \theta+1}-\frac{\lambda}{\lambda_{0}}\left(\left(c^{+}\right)^{2 \theta}-c_{\infty}^{2 \theta}\right),
$$

for some $\tilde{\mu}>0$. Arguing as in Lemma 6.3 we have

$$
\tilde{\mu} \limsup _{t \rightarrow+\infty}\left\|w^{+}(t)\right\|_{H^{1}(\mathbb{R})}^{2} \leq \frac{1}{\lambda_{0}}\left(c_{\infty}-\lambda\right)\left(\left(c^{+}\right)^{2 \theta}-c_{\infty}^{2 \theta}\right)+O\left(\left|\left(c^{+}\right)^{2 \theta}-c_{\infty}^{2 \theta}\right|^{2}\right) .
$$

From this inequality and the bound $\left|c^{+}-c_{\infty}\right| \leq K \varepsilon$ we get

$$
\left(\frac{c^{+}}{c_{\infty}}\right)^{2 \theta}-1 \geq \tilde{\mu} \limsup _{t \rightarrow+\infty}\left\|w^{+}(t)\right\|_{H^{1}(\mathbb{R})}^{2},
$$

as desired.

Proof of Theorem 1.3. In this section we prove (1-35), which implies there is no pure soliton at infinity. This will require several additional arguments, including the fundamental Lemma 7.5 and a monotonicity formula that implies that any such soliton would have polynomial decay and be $L^{1}$-integrable, in contradiction with the change of scaling.

Suppose, for a contradiction, that (1-35) is false. Then

$$
\lim _{t \rightarrow+\infty}\left\|w^{+}(t)\right\|_{H^{1}(\mathbb{R})}=0 .
$$


This, together with subcriticality, implies that $E^{+}=0$. Therefore, by using (7-1), and after some basic algebraic manipulations we see that $c^{+}$must satisfy the algebraic equation

$$
\left(c^{+}\right)^{\lambda_{0}}\left(c^{+}-\frac{\lambda}{\lambda_{0}}\right)^{1-\lambda_{0}}=2^{p}\left(1-\frac{\lambda}{\lambda_{0}}\right)^{1-\lambda_{0}}
$$

(compare with (4-17)). This relation and the uniqueness of $c_{\infty}$ give

$$
c^{+}=c_{\infty}(\lambda)
$$

In other words, the soliton solution is pure (cf. Definition 1.0).

Now consider the decomposition result for $u(t)$ from Lemma 6.2. We claim that $z(t)$ also vanishes at infinity. Indeed, from Lemma 6.2, the fact that

$$
u(t)=R(t)+z(t)=w^{+}(t)+2^{-1 /(m-1)} Q_{c_{\infty}}\left(\cdot-\rho_{2}(t)\right) \quad \text { for } t \geq t_{1},
$$

and the estimates (6-11) and (6-36), we have

$$
\lim _{t \rightarrow+\infty}\|z(t)\|_{H^{1}(\mathbb{R})}=0,
$$

$\lim _{t \rightarrow+\infty} u\left(t, \cdot+\rho_{2}(t)\right)=2^{-1 /(m-1)} Q_{c_{\infty}}$ in $H^{1}(\mathbb{R})$, and $\lim _{t \rightarrow+\infty} \rho_{2}^{\prime}(t)-\left(c_{\infty}(\lambda)-\lambda\right)=0$.

Lemma 7.1 (Monotony of mass backwards in time). Suppose $u(t)$ solution of (1-15) constructed in Theorem 3.1, satisfying (6-2) and (6-3). Define

$$
\mu[u](t):=\int_{\mathbb{R}} \frac{u^{2}(t, x)}{a_{\varepsilon}(x)} d x .
$$

Then, under the additional hypothesis $\lambda>0$ for $m=2,3,4$, we have, for all $t, t^{\prime} \geq t_{1}$ with $t^{\prime} \geq t$,

$$
\mathcal{M}[u](t)-\mathcal{M}[u]\left(t^{\prime}\right) \leq K e^{-\varepsilon \gamma t} .
$$

Proof. A simple computation tell us that the time derivative of $\mathcal{M}[u](t)$ is given by

$$
\partial_{t} \int_{\mathbb{R}} \frac{u^{2}}{a_{\varepsilon}}=2 \varepsilon \int_{\mathbb{R}} u_{x}^{2} \frac{a_{\varepsilon}^{\prime}}{a_{\varepsilon}^{2}}+\varepsilon \int_{\mathbb{R}} u^{2}\left(\lambda \frac{a_{\varepsilon}^{\prime}}{a_{\varepsilon}^{2}}-\varepsilon^{2}\left(\frac{a_{\varepsilon}^{\prime}}{a_{\varepsilon}^{2}}\right)^{\prime \prime}\right)-2 \varepsilon \int_{\mathbb{R}} \frac{a_{\varepsilon}^{\prime}}{a_{\varepsilon}} u^{m+1} .
$$

Replacing $u$ by $R+z$ (see Lemma 6.2) and using assumption (1-14) and estimates similar to (A-13), plus the smallness of $\|z(t)\|_{H^{1}(\mathbb{R})}$, we get

$$
\partial_{t} \mathcal{M}[u](t) \geq-K \varepsilon e^{-\varepsilon \gamma t},
$$

for some $K, \gamma>0$. The result follows after integration.

Remark. The estimate (7-5) is valid under the additional assumption $0<\lambda \leq \lambda_{0}$. This extra hypothesis unfortunately does not hold in the case $m=3, \lambda=0$.

Lemma 7.1 allows us to prove a version of Theorem 3.1 for positive times. 
Proposition 7.2 (Backward uniqueness). Suppose $m=2,3,4$. Let $\beta \in \mathbb{R}$ and $0<\lambda \leq \lambda_{0}$. There exist constants $K, \gamma, \varepsilon_{0}>0$ and a unique solution $v=v_{\beta} \in C\left(\left[\frac{1}{2} T_{\varepsilon},+\infty\right), H^{1}(\mathbb{R})\right)$ of $(1-15)$ such that

$$
\lim _{t \rightarrow+\infty}\left\|v(t)-2^{-1 /(m-1)} Q_{c_{\infty}}\left(\cdot-\left(c_{\infty}(\lambda)-\lambda\right) t-\beta\right)\right\|_{H^{1}(\mathbb{R})}=0
$$

Furthermore, for all $t \geq \frac{1}{2} T_{\varepsilon}$ and $s \geq 1$ the function $v(t)$ satisfies

$$
\left\|v(t)-2^{-1 /(m-1)} Q_{c_{\infty}}\left(\cdot-\left(c_{\infty}(\lambda)-\lambda\right) t-\beta\right)\right\|_{H^{s}(\mathbb{R})} \leq K \varepsilon^{-1} e^{-\varepsilon \gamma t} .
$$

Finally, suppose that there exists $\tilde{v}(t) \in H^{1}(\mathbb{R})$ solution of (1-15) such that

$$
\lim _{t \rightarrow+\infty}\left\|\tilde{v}(t)-2^{-1 /(m-1)} Q_{c_{\infty}}\left(\cdot-\rho_{2}(t)\right)\right\|_{H^{1}(\mathbb{R})}=0 .
$$

Then $\tilde{v} \equiv v_{\beta}$ for some $\beta \in \mathbb{R}$.

Proof. Given $\beta \in \mathbb{R}$, the proof of existence and uniqueness of the solution $v_{\beta}$ satisfying (7-6) and (7-7) is identical to the proof of Theorem 3.1 in Section 3 and Section A.1. First we construct a sequence of functions $v_{n}$ as in (A-1) for times $t \sim T_{n}$. Next, we prove a decomposition lemma as in Lemma A.1.4. This yields a version of (7-5) for $\mathcal{M}\left[v_{n}\right](t)$. The main difference is given in the estimates (A-14) and (A-15), where now we introduce the modified mass $\mathcal{M}\left[v_{n}\right](t)$ defined in (7-4). The energy functional in (A-18) is now given by $E_{a}\left[v_{n}\right](t)+\left(c_{\infty}(\lambda)-\lambda\right) M\left[v_{n}\right](t)$. The rest of the proof, including the uniqueness, adapts mutatis mutandis.

Now consider a solution $\tilde{v}$ of (1-15) satisfying (7-8). Using monotonicity arguments, similar to the proof of Proposition A.1.7, we show the existence of $\beta \in \mathbb{R}$ such that

$$
\left\|\tilde{v}(t)-2^{-1 /(m-1)} Q_{c_{\infty}}\left(\cdot-\left(c_{\infty}(\lambda)-\lambda\right) t-\beta\right)\right\|_{H^{1}(\mathbb{R})} \leq K \varepsilon^{-1} e^{-\varepsilon \gamma t},
$$

for some $K, \gamma>0$. This implies that there exists $\beta \in \mathbb{R}$ such that $\tilde{v}$ satisfies (7-6). The conclusion follows from the uniqueness of $v(t)$.

As a consequence of this result together with (7-3), the solution $u(t)$ constructed in Theorem 3.1 satisfies the following exponential decay at infinity: there exist $K, \gamma>0$ and $\beta \in \mathbb{R}$ such that, for all $t \geq t_{1}$, if $\tilde{\rho}_{2}(t):=\left(c_{\infty}(\lambda)-\lambda\right) t+\beta$, then

$$
\tilde{z}(t):=u(t)-2^{-1 /(m-1)} Q_{c_{\infty}}\left(\cdot-\tilde{\rho}_{2}(t)\right), \quad \text { satisfies } \quad\|\tilde{z}(t)\|_{H^{2}(\mathbb{R})} \leq K \varepsilon^{-1} e^{-\varepsilon \gamma t} .
$$

Now we prove that this strong $H^{1}$-convergence gives rise to strange localization properties.

Lemma 7.3 ( $L^{2}$-exponential decay on the left). There exist $K, \tilde{x}_{0}>0$ large enough such that for all $t \geq T_{0}$ and for all $x_{0} \geq \tilde{x}_{0}$

$$
\left\|u\left(t, \cdot+\tilde{\rho}_{2}(t)\right)\right\|_{L^{2}\left(x \leq-x_{0}\right)}^{2} \leq K e^{-x_{0} / K} .
$$

Proof. Suppose $x_{0}>0, t, t_{0} \geq t_{1}$ and $\sigma>0$ from (6-3). Consider the modified mass

$$
\tilde{I}_{t_{0}, x_{0}}(t):=\frac{1}{2} \int_{\mathbb{R}} \frac{u^{2}(t, x)}{a_{\varepsilon}(x)}(1-\phi(y)) d x,
$$


with $y:=x-\left(\tilde{\rho}_{2}\left(t_{0}\right)+\sigma\left(t-t_{0}\right)-x_{0}\right)$ and $\phi$ defined in (6-30). For this quantity we claim that for $x_{0}>\tilde{x}_{0}$ and for all $t \geq t_{0}$,

$$
\tilde{I}_{t_{0}, x_{0}}\left(t_{0}\right)-\tilde{I}_{t_{0}, x_{0}}(t) \leq K e^{-x_{0} / K}\left(1+e^{-\frac{1}{2} \sigma\left(t-t_{0}\right) / K}\right) .
$$

Let us assume this result for a moment. After taking the limit $t \rightarrow+\infty$ and using (6-3), we have $\lim _{t \rightarrow+\infty} \tilde{I}_{t_{0}, x_{0}}(t)=0$ and thus

$$
\tilde{I}_{t_{0}, x_{0}}\left(t_{0}\right) \leq K e^{-x_{0} / K} .
$$

Now (7-10) follows from the fact that $t_{0} \geq t_{1}$ is arbitrary.

Finally, let us prove (7-11). A direct calculation tell us that

$$
\begin{aligned}
\frac{1}{2} \partial_{t} \int_{\mathbb{R}} \frac{(1-\phi(y))}{a_{\varepsilon}} u^{2}=\frac{3}{2} \int_{\mathbb{R}} \frac{\phi^{\prime}}{a_{\varepsilon}} u_{x}^{2}+\frac{3}{2} \varepsilon \int_{\mathbb{R}} \frac{a_{\varepsilon}^{\prime}}{a_{\varepsilon}^{2}}(1-\phi) u_{x}^{2}-\frac{m}{m+1} \int_{\mathbb{R}} \phi^{\prime} u^{m+1} \\
+\frac{1}{2} \int_{\mathbb{R}} u^{2}\left((\sigma+\lambda) \frac{\phi^{\prime}}{a_{\varepsilon}}-\frac{\phi^{(3)}}{a_{\varepsilon}}+3 \varepsilon \phi^{\prime \prime} \frac{a_{\varepsilon}^{\prime}}{a_{\varepsilon}^{2}}+3 \varepsilon^{2} \phi^{\prime}\left(\frac{a_{\varepsilon}^{\prime}}{a_{\varepsilon}^{2}}\right)^{\prime}\right) \\
+\frac{\varepsilon}{2} \int_{\mathbb{R}} u^{2}\left(\lambda \frac{a_{\varepsilon}^{\prime}}{a_{\varepsilon}^{2}}-\varepsilon^{2}\left(\frac{a_{\varepsilon}^{\prime}}{a_{\varepsilon}^{2}}\right)^{\prime \prime}\right)(1-\phi)-\varepsilon \int_{\mathbb{R}} \frac{a_{\varepsilon}^{\prime}}{a_{\varepsilon}} u^{m+1}(1-\phi) .
\end{aligned}
$$

Using (7-9), we have

$$
\begin{aligned}
\left|\int_{\mathbb{R}} \phi^{\prime} u^{m+1}\right| & \leq K \varepsilon^{(m-1) / 2} \int_{\mathbb{R}} \phi^{\prime} \tilde{z}^{2}+K e^{-\frac{1}{2} \sigma\left(t-t_{0}\right)} e^{-x_{0} / K}, \\
\left|\int_{\mathbb{R}} \frac{a_{\varepsilon}^{\prime}}{a_{\varepsilon}} u^{m+1}(1-\phi)\right| & \leq K e^{-\frac{1}{2} \sigma\left(t-t_{0}\right)} e^{-x_{0} / K}+K \varepsilon^{(m-1) / 2} \int_{\mathbb{R}} \frac{a_{\varepsilon}^{\prime}}{a_{\varepsilon}} \tilde{z}^{2}(1-\phi) .
\end{aligned}
$$

After this, it is easy to conclude that

$$
\frac{1}{2} \partial_{t} \int_{\mathbb{R}} \frac{(1-\phi(y))}{a_{\varepsilon}} u^{2} \geq-K e^{-\frac{1}{2} \sigma\left(t-t_{0}\right)} e^{-x_{0} / K} .
$$

The conclusion follows after integration in time.

The proof of decay on the right-hand side of the soliton requires more care, and is valid under the assumption $\lim \sup _{t \rightarrow+\infty}\left\|w^{+}(t)\right\|_{H^{1}(\mathbb{R})}=0$ and $\lambda>0$. We do not expect to have exponential decay in a general situation, but for our purposes we only need a polynomial decay. The following result is due to Y. Martel.

Lemma 7.4 ( $L^{2}$-polynomial decay on the right the soliton solution). There exist $K, \tilde{x}_{0}>0$ large enough but independent of $\varepsilon$, such that for all $t \geq T_{0}$ and for all $x_{0} \geq \tilde{x}_{0}$

$$
\int_{\mathbb{R}}\left(x-x_{0}\right)_{+}^{2} \tilde{z}^{2}\left(t, x+\tilde{\rho}_{2}(t)\right) d x \leq K,
$$

where $x_{+}:=\max \{x, 0\}$.

Proof. Take $x_{0}>0, t_{0}, t \geq t_{1}$ and define

$$
\hat{I}_{t_{0}, x_{0}}(t):=\int_{\mathbb{R}} \tilde{z}^{2}(t, x) \phi(\tilde{y}) d x ; \quad \tilde{y}:=x-\left(\tilde{\rho}_{2}\left(t_{0}\right)+\tilde{\sigma}\left(t-t_{0}\right)+x_{0}\right),
$$


and

$$
\hat{J}_{t_{0}, x_{0}}(t):=\int_{\mathbb{R}} \tilde{z}_{x}^{2}(t, x) \phi(\tilde{y}) d x
$$

Here $\phi$ is the cut-off function defined in (6-30), and $\tilde{\sigma}$ is a fixed constant satisfying $\tilde{\sigma}>2\left(c_{\infty}(\lambda)-\lambda\right)$. We claim that there exists $K>0$ such that (for simplicity we omit the dependence if no confusion is present)

$$
\left|\partial_{t} \hat{I}_{t_{0}, x_{0}}(t)\right| \leq K \int_{\mathbb{R}}\left(\tilde{z}_{x}^{2}+\tilde{z}^{2}\right)\left[\phi^{\prime}+\varepsilon a^{\prime}(\varepsilon x) \phi\right] d x+K\|\tilde{z}(t)\|_{H^{1}(\mathbb{R})} e^{-\varepsilon\left(t-t_{0}\right) / K} e^{-\varepsilon x_{0} / K},
$$

and

$$
\left|\partial_{t} \hat{J}_{t_{0}, x_{0}}(t)\right| \leq K \int_{\mathbb{R}}\left(\tilde{z}_{x x}^{2}+\tilde{z}_{x}^{2}+\tilde{z}^{2}\right)\left[\phi^{\prime}+\varepsilon a^{\prime}(\varepsilon x) \phi\right] d x+K\|\tilde{z}(t)\|_{H^{2}(\mathbb{R})} e^{-\varepsilon\left(t-t_{0}\right) / K} e^{-\varepsilon x_{0} / K}
$$

Indeed, these estimates are proved in the same way as in Lemma 6.4 and Section A.4. For the sake of brevity we skip the details.

From Proposition 7.2 and the exponential decay of $z$ we have that both right-hand sides in (7-12)-(7-13) are integrable between $t_{0}$ and $+\infty$. We get

$$
\hat{I}_{t_{0}, x_{0}}\left(t_{0}\right) \leq K \int_{t_{0}}^{+\infty} \int_{\mathbb{R}}\left(\tilde{z}_{x}^{2}+\tilde{z}^{2}\right)\left[\phi^{\prime}+\varepsilon a^{\prime}(\varepsilon x) \phi\right] d x d t+K \varepsilon^{-1} \sup _{t \geq t_{0}}\|\tilde{z}(t)\|_{H^{1}(\mathbb{R})} e^{-\varepsilon x_{0} / K} .
$$

In the same line, we have

$$
\hat{J}_{t_{0}, x_{0}}\left(t_{0}\right) \leq K \int_{t_{0}}^{+\infty} \int_{\mathbb{R}}\left(\tilde{z}_{x x}^{2}+\tilde{z}_{x}^{2}+\tilde{z}^{2}\right)\left[\phi^{\prime}+\varepsilon a^{\prime}(\varepsilon x) \phi\right] d x d t+K \varepsilon^{-1} \sup _{t \geq t_{0}}\|\tilde{z}(t)\|_{H^{2}(\mathbb{R})} e^{-\varepsilon x_{0} / K} .
$$

Note that both quantities above are integrable with respect to $x_{0}$.

Set $\xi_{0}(\tilde{y}):=\phi(\tilde{y})$ and $\xi_{j}(\tilde{y}):=\int_{-\infty}^{\tilde{y}} \xi_{j-1}(s) d s$, for $j=1,2$. Recall that the $\xi_{j}$ are positive and increasing functions on $\mathbb{R}$, with $\xi_{j}(\tilde{y}) \rightarrow 0$ as $\tilde{y} \rightarrow-\infty$, and $\xi_{j}(\tilde{y})-\tilde{y}^{j} \rightarrow 0$ as $\tilde{y} \rightarrow+\infty$. Integrating (7-14) from $x_{0}$ to $+\infty$ and using Fubini's theorem we obtain

$$
\int_{\mathbb{R}} \xi_{1}\left(\tilde{y}\left(t_{0}\right)\right) \tilde{z}^{2}\left(t_{0}\right) \leq K \int_{t_{0}}^{+\infty} \int_{\mathbb{R}}\left(\tilde{z}_{x}^{2}+\tilde{z}^{2}\right)\left[\xi_{0}+\varepsilon a^{\prime}(\varepsilon x) \xi_{1}\right]+K \varepsilon^{-2} \sup _{t \geq t_{0}}\|\tilde{z}(t)\|_{H^{1}(\mathbb{R})} e^{-\varepsilon x_{0} / K} .
$$

Similarly, from (7-15),

$$
\int_{\mathbb{R}} \xi_{1}\left(\tilde{y}\left(t_{0}\right)\right) \tilde{z}_{x}^{2}\left(t_{0}, x\right) d x \leq K \varepsilon^{-3} e^{-2 \varepsilon \gamma t_{0}}+K \varepsilon^{-2} \sup _{t \geq t_{0}}\|\tilde{z}(t)\|_{H^{2}(\mathbb{R})} e^{-\varepsilon x_{0} / K} .
$$

In conclusion, thanks to the exponential decay of $\tilde{z}$ and (7-16)-(7-17), we have

$$
\int_{t_{0}}^{+\infty} \int_{\mathbb{R}} \xi_{1}\left(x-\tilde{\rho}_{2}(t)-x_{0}\right)\left(\tilde{z}_{x}^{2}+\tilde{z}^{2}\right)(t, x) d x d t<+\infty .
$$

Furthermore, $\tilde{\rho}_{2}(t) \leq \tilde{\rho}_{2}\left(t_{0}\right)+\sigma\left(t-t_{0}\right)$ for all $t \geq t_{0}$. Thus

$$
\int_{t_{0}}^{+\infty} \int_{\mathbb{R}} \xi_{1}(\tilde{y}(t))\left(\tilde{z}_{x}^{2}+\tilde{z}^{2}\right)(t, x) d x d t<+\infty .
$$


In addition, an easier calculation gives

$$
\int_{t_{0}}^{+\infty} \int_{\mathbb{R}} a^{\prime}(\varepsilon x) \xi_{2}(\tilde{y}(t))\left(\tilde{z}_{x}^{2}+\tilde{z}^{2}\right)(t, x) d x d t<+\infty
$$

From (7-18) and (7-19), we can perform a second integration with respect to $x_{0}$ in (7-16) to obtain

$$
\int_{\mathbb{R}} \xi_{2}\left(\tilde{y}\left(t_{0}\right)\right) \tilde{z}^{2}\left(t_{0}, x\right) d x \leq K(\varepsilon),
$$

uniformly for $x_{0}$ large. Since $t_{0}$ is arbitrary, this last estimate gives the conclusion.

Lemma 7.5 ( $L^{1}$-integrability and smallness). Assume (7-3) holds. There exist $K, T_{0}>0$ large enough such that $u\left(t, \cdot+\tilde{\rho}_{2}(t)\right) \in L^{1}(\mathbb{R})$ for all $t \geq T_{0}$. Moreover,

$$
\left|\int_{\mathbb{R}} z(t)\right| \leq \frac{1}{100}
$$

Finally, from the $L^{1}$ conservation law (1-9), we have $u(t) \in L^{1}(\mathbb{R})$ for all $t \in \mathbb{R}$ and

$$
\int_{\mathbb{R}} u(t)=\int_{\mathbb{R}} Q
$$

Proof. Let $x_{0} \geq \tilde{x}_{0}$ to be fixed below. If $|x| \geq x_{0}$ we have $2^{-1 /(m-1)} Q_{c_{\infty}}(x) \leq K e^{-\sqrt{c_{\infty}}|x|}$. Since $\tilde{z}\left(t, x+\tilde{\rho}_{2}(t)\right)=u\left(t, x+\tilde{\rho}_{2}(t)\right)-2^{-1 /(m-1)} Q_{c_{\infty}}(x)$, by using Lemma 7.3 and the stability bound (6-2), in addition to a Gagliardo-Nirenberg type inequality, we then get, for all $x \leq-x_{0}$,

$$
\left|\tilde{z}\left(t, x+\tilde{\rho}_{2}(t)\right)\right| \leq K\left\|\tilde{z}\left(t, \cdot+\tilde{\rho}_{2}(t)\right)\right\|_{L^{2}(y \geq x)}^{\frac{1}{2}}\left\|\tilde{z}_{y}\left(t, \cdot+\tilde{\rho}_{2}(t)\right)\right\|_{L^{2}(\mathbb{R})}^{\frac{1}{2}} \leq K \varepsilon^{1 / 4} e^{x / K},
$$

For $x \in\left[-x_{0}, x_{0}\right]$ one has

$$
\int_{\left[-x_{0}, x_{0}\right]} \tilde{z}\left(t, x+\tilde{\rho}_{2}(t)\right) \leq K x_{0}^{1 / 2}\left\|\tilde{z}\left(t, x+\tilde{\rho}_{2}(t)\right)\right\|_{L^{2}(\mathbb{R})}^{1 / 2} \leq K x_{0}^{1 / 2} \varepsilon^{1 / 4} .
$$

The case $x \geq x_{0}$ requires more care. From Lemma 7.4 and the Cauchy-Schwarz inequality, we have (for clarity we drop the dependence on $\left.x+\tilde{\rho}_{2}(t)\right)$

$$
\left|\int_{x \geq x_{0}} z(t)\right| \leq \frac{K}{\left(x_{0}-\tilde{x}_{0}\right)^{1 / 2-}}\left(\int_{x \geq x_{0}}\left(1+\left(x-\tilde{x}_{0}\right)^{2}\right) z^{2}(t)\right)^{1 / 2} \leq \frac{K}{x_{0}^{1 / 2-}},
$$

for $x_{0}$ large enough, independent of $\varepsilon$. From these estimates we obtain the smallness condition (7-20).

That $u(t)$ is in $L^{1}(\mathbb{R})$ for all $t \in \mathbb{R}$ is a consequence of Proposition 2.1. It is clear that from this last fact (1-9) remains constant for all time and (7-21) holds.

Conclusion of the proof. From (7-2) and Section A.6 we have

$$
\lim _{t \rightarrow+\infty} \int_{\mathbb{R}} z(t)=\left(1-\frac{\left(c^{+}\right)^{\theta-\frac{1}{4}}}{2^{1 /(m-1)}}\right) \int_{\mathbb{R}} Q=\left(1-\kappa_{m}\right) \int_{\mathbb{R}} Q \neq 0, \quad \text { with } \quad \kappa_{m}:=\frac{c_{\infty}^{\frac{3-m}{2(m-1)}}}{2^{1 /(m-1)}},
$$


For $m=3,4$ it is easy to see that $1-\kappa_{m}>\frac{1}{10}$. For $m=2$ we have $\kappa_{2}=\frac{1}{2} c_{\infty}^{1 / 2}$; but from (4-19) we know that $c_{\infty} \leq 2^{\frac{4}{3}}$. In any case, then, we have $1-\kappa_{m}>\frac{1}{10}$. Thus

$$
\left|\lim _{t \rightarrow+\infty} \int_{\mathbb{R}} z(t)\right| \geq \frac{1}{10} \int_{\mathbb{R}} Q
$$

in contradiction with (7-20). This finishes the proof of (1-35).

\section{Appendix: Proofs of auxiliary results}

A.1. Sketch of the proof of Theorem 3.1. We follow [Martel 2005], to which we refer the reader for all the details omitted here.

Let $\left(T_{n}\right)_{n \in \mathbb{N}} \subseteq \mathbb{R}$ be an increasing sequence with $T_{n} \geq \frac{1}{2} T_{\varepsilon}$ for all $n$ and $\lim _{n \rightarrow+\infty} T_{n}=+\infty$. For notational simplicity we denote by $\tilde{T}_{n}$ the sequence $(1-\lambda) T_{n}$. Consider the solution $u_{n}(t)$ of the Cauchy problem

$$
\left\{\begin{array}{l}
\left(u_{n}\right)_{t}+\left(\left(u_{n}\right)_{x x}-\lambda u_{n}+a_{\varepsilon} u_{n}^{m}\right)_{x}=0 \quad \text { in } \mathbb{R}_{t} \times \mathbb{R}_{x} \\
u_{n}\left(-T_{n}\right)=Q\left(\cdot-\tilde{T}_{n}\right)
\end{array}\right.
$$

Thus $u_{n}$ is a solution of (aKdV) that at time $t=-T_{n}$ corresponds to the soliton $Q\left(\cdot-\tilde{T}_{n}\right)$. Clearly, $Q\left(\cdot-\tilde{T}_{n}\right) \in H^{s}(\mathbb{R})$ for every $s \geq 0$; moreover, there exists a uniform constant $C=C(s)>0$ such that

$$
\left\|Q\left(\cdot-\tilde{T}_{n}\right)\right\|_{H^{s}(\mathbb{R})} \leq C .
$$

According to Propositions 2.1 and 2.2, $u_{n}$ is locally well defined in time, and global for positive times in $H^{1}(\mathbb{R})$. Let $I_{n}$ be its maximal interval of existence.

The next step is to establish uniform estimates starting from a fixed time $t=-\frac{1}{2} T_{\varepsilon}<0$ so negative that the soliton is not influenced by the perturbation in the potential. That is the content of this proposition, proved in the next section:

Proposition A.1.1 (Uniform estimates in $H^{s}$ for large times). There exist constants $K, \gamma>0$ and $\varepsilon_{0}>0$ such that, for all $0<\varepsilon<\varepsilon_{0}$ and for all $n \in \mathbb{N}$ we have $\left[-T_{n},-\frac{1}{2} T_{\varepsilon}\right] \subseteq I_{n}\left(\operatorname{so~} u_{n} \in C\left(\left[-T_{n},-\frac{1}{2} T_{\varepsilon}\right], H^{s}(\mathbb{R})\right)\right)$ and, for all $t \in\left[-T_{n},-\frac{1}{2} T_{\varepsilon}\right]$,

$$
\left\|u_{n}(t)-Q(\cdot-(1-\lambda) t)\right\|_{H^{s}(\mathbb{R})} \leq K \varepsilon^{-1} e^{\gamma \varepsilon t} .
$$

In particular, there exists a constant $C_{s}>0$ such that, for all $t \in\left[-T_{n},-\frac{1}{2} T_{\varepsilon}\right]$,

$$
\left\|u_{n}(t)\right\|_{H^{s}(\mathbb{R})} \leq C_{s} .
$$

Using this result we will obtain the existence of a critical element $u_{0, *} \in H^{s}(\mathbb{R})$, with good compact properties, nondispersive, and uniformly close to the desired soliton.

Indeed, consider the sequence $\left(u_{n}\left(-\frac{1}{2} T_{\varepsilon}\right)\right)_{n \in \mathbb{N}} \subseteq H^{s}(\mathbb{R})$. A standard argument shows that, given any $\delta>0$, there exist $\varepsilon_{0}>0$ and $K_{0}>0$ such that

$$
\int_{|x|>K_{0}} u_{n}^{2}\left(-\frac{1}{2} T_{\varepsilon}\right)<\delta \text { for all } 0<\varepsilon<\varepsilon_{0} \text { and } n \in \mathbb{N} \text {. }
$$


To use this in the proof of Theorem 3.1, note first that (A-3) implies that

$$
\left\|u_{n}\left(-T_{\varepsilon} / 2\right)\right\|_{H^{1}(\mathbb{R})} \leq C_{0},
$$

independently of $n$. Thus, up to a subsequence we may suppose that $u_{n}\left(-\frac{1}{2} T_{\varepsilon}\right) \rightarrow u_{*, 0}$ in the $H^{1}(\mathbb{R})$ weak sense, and $u_{n}\left(-\frac{1}{2} T_{\varepsilon}\right) \rightarrow u_{*, 0}$ in $L_{l o c}^{2}(\mathbb{R})$, as $n \rightarrow+\infty$. In addition, from (A-4) we have strong convergence in $L^{2}(\mathbb{R})$. From interpolation and the bound (A-3) we have strong convergence in $H^{s}(\mathbb{R})$ for any $s \geq 1$.

Let $u_{*}=u_{*}(t)$ be the solution of (1-1) with initial data $u_{*}\left(-\frac{1}{2} T_{\varepsilon}\right)=u_{*, 0}$. From Proposition 2.1 we have $u_{*} \in C\left(I, H^{s}(\mathbb{R})\right)$, where $-\frac{1}{2} T_{\varepsilon} \in I$, the corresponding maximal interval of existence. Thus, using the continuous dependence of $u_{n}$ and $u_{*}$, we obtain $u_{n}(t) \rightarrow u_{*}(t)$ in $H^{s}(\mathbb{R})$ for every $t \leq-\frac{1}{2} T_{\varepsilon} \subseteq I$. Passing to the limit in (A-2) we obtain, for all $t \leq-\frac{1}{2} T_{\varepsilon}$,

$$
\left\|u_{*}(t)-Q(\cdot-(1-\lambda) t)\right\|_{H^{s}(\mathbb{R})} \leq K \varepsilon^{-1} e^{\varepsilon \gamma t},
$$

as desired. This completes the proof of the existence part of Theorem 3.1, assuming Proposition A.1.1. A.1.2. Uniform $H^{1}$ estimates. Next we outline the proof of Proposition A.1.1. We consider only the $H^{1}$ case. The first step in the proof is the following bootstrap property:

Proposition A.1.3 (Uniform estimates with and without decay assumption). Let $m=2,3,4$ and $0 \leq \lambda \leq$ $\lambda_{0}<1$. There exist constants $K, \gamma, \varepsilon_{0}>0$ such that for all $0<\varepsilon<\varepsilon_{0}$ the following is true.

(1) Suppose $m=3$, or $m=2,4$ with $\lambda>0$. Then there exists $\alpha_{0}>0$ such that, for all $0<\alpha<\alpha_{0}$, if for some $-T_{n, *} \in\left[-T_{n},-\frac{1}{2} T_{\varepsilon}\right]$ and for all $t \in\left[-T_{n},-T_{n, *}\right]$ we have

$$
\left\|u_{n}(t)-Q(\cdot-(1-\lambda) t)\right\|_{H^{1}(\mathbb{R})} \leq 2 \alpha,
$$

then, for all $t \in\left[-T_{n},-T_{n, *}\right]$,

$$
\left\|u_{n}(t)-Q(\cdot-(1-\lambda) t)\right\|_{H^{1}(\mathbb{R})} \leq K \varepsilon^{-1} e^{\varepsilon \gamma t} .
$$

(2) Now suppose $m=2,4$ and $\lambda=0$. Then (A-6) holds if for some $-T_{n, *} \in\left[-T_{n},-\frac{1}{2} T_{\varepsilon}\right]$ and for all $t \in\left[-T_{n},-T_{n, *}\right]$ one has

$$
\left\|u_{n}(t)-Q(\cdot-(1-\lambda) t)\right\|_{H^{1}(\mathbb{R})} \leq 2 K \varepsilon^{-1} e^{\varepsilon \gamma t} .
$$

Proof of Proposition A.1.1, assuming the validity of Proposition A.1.3. We assume item (1) of the proposition. The case in which we assume item (2) is similar. From (A-1) we have

$$
\left\|u_{n}\left(-T_{n}\right)-Q\left(-(1-\lambda) T_{n}\right)\right\|_{H^{1}(\mathbb{R})}=0,
$$

so there exists $t_{0}=t_{0}(n, \alpha)>0$ such that (A-5) holds true for all $t \in\left[-T_{n},-T_{n}+t_{0}\right]$. Now consider (we adopt the convention $T_{*, n}>0$ )

$$
-\tilde{T}_{*, n}:=\sup \left\{t \in\left[-T_{n},-\frac{1}{2} T_{\varepsilon}\right]:\left\|u_{n}\left(t^{\prime}\right)-Q\left(\cdot t-(1-\lambda) t^{\prime}\right)\right\|_{H^{1}(\mathbb{R})} \leq 2 \alpha \text { for all } t^{\prime} \in\left[-T_{n}, t\right]\right\} .
$$


Assume, for a contradiction, that $-\tilde{T}_{*, n}<-\frac{1}{2} T_{\varepsilon}$. From Proposition A.1.3, we have

$$
\left\|u_{n}\left(t^{\prime}\right)-Q\left(\cdot t-(1-\lambda) t^{\prime}\right)\right\|_{H^{1}(\mathbb{R})} \leq K \varepsilon^{-1} e^{\gamma \varepsilon t} \leq \alpha
$$

for $\varepsilon$ small enough (recall that $t \leq-\frac{1}{2} T_{\varepsilon}=-\frac{1}{2(1-\lambda)} \varepsilon^{-1-\frac{1}{100}}$ ). This contradicts the definition of $\tilde{T}_{*, n}$.

We turn to the proof of Proposition A.1.3. The first step is to decompose the solution preserving a standard orthogonality condition. To obtain this, we suppose (without loss of generality, by taking $T_{n, *}$ even larger) that, for all $t \in\left[-T_{n},-T_{n, *}\right]$,

$$
\left\|u_{n}(t)-Q\left(\cdot-(1-\lambda) t-r_{n}(t)\right)\right\|_{H^{1}(\mathbb{R})} \leq 2 \alpha,
$$

for all smooth $r_{n}=r_{n}(t)$ satisfying $r_{n}\left(-T_{n}\right)=0$ and $\left|r_{n}^{\prime}(t)\right| \leq 1 / t^{2}$. A posteriori we will prove that this condition can be improved and extended to any time $t \in\left[-T_{n},-\frac{1}{2} T_{\varepsilon}\right]$.

In what follows we drop the index $n$ in $-T_{*, n}$ and $u_{n}$, if no confusion can arise.

Lemma A.1.4 (Modulation). There exist $K, \gamma, \varepsilon_{0}>0$ and a unique $C^{1}$ function $\rho_{0}:\left[-T_{n},-T_{*}\right] \rightarrow \mathbb{R}$ such that for all $0<\varepsilon<\varepsilon_{0}$ the function $z$ defined by

$$
z(t, x):=u(t, x)-R(t, x) ; \quad R(t, x):=Q\left(x-(1-\lambda) t-\rho_{0}(t)\right)
$$

satisfies, for all $t \in\left[-T_{n},-T_{*}\right]$,

$$
\int_{\mathbb{R}} z(t, x) R_{x}(t, x) d x=0, \quad\|z(t)\|_{H^{1}(\mathbb{R})} \leq K \alpha, \quad \rho_{0}\left(-T_{n}\right)=0 .
$$

Moreover, z satisfies the modified $g K d V$ equation

$$
z_{t}+\left(z_{x x}-\lambda z+a_{\varepsilon}\left((R+z)^{m}-R^{m}\right)+\left(1-a_{\varepsilon}\right) R^{m}\right)_{x}-\rho_{0}^{\prime}(t) R_{x}=0,
$$

and

$$
\left|\rho_{0}^{\prime}(t)\right| \leq K\left(e^{\varepsilon \gamma t}+\|z(t)\|_{H^{1}(\mathbb{R})}+\|z(t)\|_{L^{2}(\mathbb{R})}^{2}\right) .
$$

Proof of Lemma A.1.4. The proof of (A-10) is a standard consequence of the implicit function theorem, the definition of $T_{*}\left(=T_{*, n}\right)$, and the definition of $u_{n}\left(-T_{n}\right)$ given in (A-1). Similarly, the proof of (A-11) follows after a simple computation.

Now we deal with (A-12). Taking the time derivative of (A-9) and using (A-11), we get

$$
\begin{aligned}
0 & =\int_{\mathbb{R}} z_{t} R_{x}-\left(1-\lambda+\rho_{0}^{\prime}\right) \int_{\mathbb{R}} z R_{x x} \\
& =\int_{\mathbb{R}}\left(z_{x x}-z+a_{\varepsilon}\left((R+z)^{m}-R^{m}\right)+\left(1-a_{\varepsilon}\right) R^{m}\right) R_{x x}+\rho_{0}^{\prime} \int_{\mathbb{R}} R_{x}\left(R_{x}+z_{x}\right) .
\end{aligned}
$$

Note that

$$
\int_{\mathbb{R}} R_{x}\left(R_{x}+z_{x}\right)=\int_{\mathbb{R}} Q^{\prime 2}+O\left(\|z(t)\|_{L^{2}(\mathbb{R})}\right) .
$$


On the other hand, from (1-13), (A-10), the uniform bound on $\rho_{0}^{\prime}(t)$ in the definition of $T_{*}$, and the exponential decay of $R$, we have

$$
\left|\int_{\mathbb{R}}\left(1-a_{\varepsilon}\right) R^{m} R_{x x}\right| \leq K e^{\varepsilon \gamma t} .
$$

Indeed, first note that from (A-8), by integrating between $-T_{n}$ and $t$ and using (A-10) we get

$$
\rho_{0}(t) \leq-\frac{1}{T_{n}}-\frac{1}{t} \leq \frac{2}{T_{\varepsilon}} \leq K \varepsilon^{1+\frac{1}{100}}
$$

Thus $t+\rho_{0}(t) \leq t+K \varepsilon^{1+\frac{1}{100}} \leq \frac{9}{10} t$. Therefore, by possibly redefining $\gamma$, we have from (1-13)

$$
\begin{aligned}
\left|\int_{\mathbb{R}}\left(1-a_{\varepsilon}\right) R^{m} R_{x x}\right| & \leq K \int_{-\infty}^{0} e^{\gamma \varepsilon x} e^{-(m+1)\left|x-\left(t+\rho_{0}(t)\right)\right|} d x+K e^{(m+1)\left(t+\rho_{0}(t)\right)} \int_{0}^{\infty} e^{-(m+1) x} d x \\
& \leq K \exp \left(\gamma \varepsilon\left(t+\rho_{0}(t)\right)\right)+K \exp \left(\gamma(m+1)\left(t+\rho_{0}(t)\right)\right) \leq K e^{\gamma \varepsilon t} .
\end{aligned}
$$

Finally,

$$
\int_{\mathbb{R}} R_{x x}\left(z_{x x}-z+a_{\varepsilon}\left((R+z)^{m}-R^{m}\right)\right)=O\left(\|z(t)\|_{L^{2}(\mathbb{R})}+\|z(t)\|_{L^{2}(\mathbb{R})}^{2}\right) .
$$

Collecting the estimates above we obtain (A-12).

A.1.5. Almost conservation of mass and energy. Recall that from the remark on page 586 that the modified mass defined in (2-8) satisfies

$$
\tilde{M}[u](t) \leq \tilde{M}[u]\left(-T_{n}\right) .
$$

for all $-T_{n} \leq t \leq-\frac{1}{2} T_{\varepsilon}$. Moreover, in the case $m=2,4$ and $\lambda=0$, since (1-20) and (A-7) hold, there exist $K, \gamma>0$ such that

$$
M[u](t) \leq M[u]\left(-T_{n}\right)+K \varepsilon e^{\gamma \varepsilon t},
$$

for $\varepsilon$ small enough. By extending the definition of $\tilde{M}[u]$ to the latter case, we have almost conservation of mass, with exponential loss for all cases.

Similarly, in the region considered the soliton $R(t)$ is an almost solution of (1-15); in particular it must conserve mass $\tilde{M}$ (2-8) and the energy $E_{a}(1-21)$, at least for large negative time. Indeed, an argument as in Lemma 6.3 (but easier) gives

$$
E_{a}[R]\left(-T_{n}\right)-E_{a}[R](t)+(1-\lambda)\left[\tilde{M}[R]\left(-T_{n}\right)-\tilde{M}[R](t)\right] \leq K e^{\gamma \varepsilon t} .
$$

for some constant $K>0$ and all time $t \in\left[-T_{n}, T_{*}\right]$

The next step is the use conservation of energy to provide control in the $R(t)$ direction (which is essential in order to obtain certain coercivity properties; see Lemma 2.3). Following Lemma 5.4, one has

$$
\left|\int_{\mathbb{R}} R z(t)\right| \leq \frac{K}{1-\lambda}\left(e^{\gamma \varepsilon t}+\|z(t)\|_{L^{2}(\mathbb{R})}^{2}+e^{\gamma \varepsilon t}\|z(t)\|_{L^{2}(\mathbb{R})}\right) .
$$

for constants $K, \gamma>0$ independent of $\varepsilon$. 
Now, consider the energy $E_{a}[u]$ and the mass $\tilde{M}[u]$ defined in (1-21) and (2-8). One has

$$
E_{a}[u](t)+(1-\lambda) \tilde{M}[u](t)=E_{a}[R](t)+(1-\lambda) \tilde{M}[R](t)-\int_{\mathbb{R}} z\left(a_{\varepsilon}-1\right) R^{m}+\mathscr{F}_{0}(t),
$$

where $\mathscr{F}_{0}$ is the quadratic functional

$$
\mathscr{F}_{0}(t):=\frac{1}{2} \int_{\mathbb{R}}\left(z_{x}^{2}+\lambda z^{2}\right)+(1-\lambda) \tilde{M}[z]-\frac{1}{m+1} \int_{\mathbb{R}} a_{\varepsilon}\left((R+z)^{m+1}-R^{m+1}-(m+1) R^{m} z\right) .
$$

In addition, for any $t \in\left[-T_{n},-T_{*}\right]$,

$$
\left|\int_{\mathbb{R}} z\left(a_{\varepsilon}-1\right) R^{m}\right| \leq K e^{\gamma \varepsilon t}\|z(t)\|_{L^{2}(\mathbb{R})} .
$$

The proof of (A-18) is essentially an expansion of the energy-mass functional using the relation $u(t)=$ $R(t)+z(t)$. The proof of $(\mathrm{A}-19)$ is similar to (A-13).

The functional $\mathscr{F}_{0}(t)$ just defined enjoys the following coercivity property: there exist $K, \lambda_{0}>0$ independent of $\varepsilon$ such that for every $t \in\left[-T_{n},-T_{*}\right]$

$$
\mathscr{F}_{0}(t) \geq \lambda_{0}\|z(t)\|_{H^{1}(\mathbb{R})}^{2}-\left|\int_{\mathbb{R}} R(t) z(t)\right|^{2}-K e^{\gamma \varepsilon t}\|z(t)\|_{L^{2}(\mathbb{R})}^{2}-K\|z(t)\|_{L^{2}(\mathbb{R})}^{3} .
$$

This bound is simply a consequence of the inequality $\lambda+(1-\lambda) a_{\varepsilon}^{1 / m}(x) \geq 1,(\mathrm{~A}-10)$ and Lemma 2.3. A.1.6. End of proof of Proposition A.1.3. Now by using (A-18), (A-20), and the estimates (A-14), (A-15), and (A-17) we finally get (A-6). Indeed, note that

$$
E_{a}[u](t)-E_{a}[u]\left(-T_{n}\right)+(1-\lambda)\left[\tilde{M}[u](t)-\tilde{M}[u]\left(-T_{n}\right)\right] \leq K e^{\varepsilon \gamma t} .
$$

On the other hand, from (A-18) and (A-10),

$$
\begin{array}{r}
E_{a}[u](t)-E_{a}[u]\left(-T_{n}\right)+(1-\lambda)\left[\tilde{M}[u](t)-\tilde{M}[u]\left(-T_{n}\right)\right] \\
\geq \mathscr{F}_{0}(t)-K e^{\gamma \varepsilon t}-K e^{\gamma \varepsilon t}\|z(t)\|_{L^{2}(\mathbb{R})}
\end{array}
$$

since $z\left(-T_{n}\right)=0$ and $\mathscr{F}_{0}\left(-T_{n}\right)=0$. Finally, from $(\mathrm{A}-20)$ and $(\mathrm{A}-17)$ we get

$$
\|z(t)\|_{H^{1}(\mathbb{R})} \leq K e^{\gamma \varepsilon t}
$$

Plugging this estimate into (A-12), we obtain that $\left|\rho_{0}^{\prime}(t)\right| \leq K e^{\gamma \varepsilon t}$, and thus after integration we get the final uniform estimate (A-6) for the $H^{1}$-case. Note that we have also improved the estimate on $\rho_{0}^{\prime}(t)$ assumed in (A-8).

We now address the uniqueness part of Theorem 3.1. Recall that the solution $u$ constructed above is in $C\left(\mathbb{R}, H^{s}(\mathbb{R})\right)$ for any $s \geq 1$, and satisfies the exponential decay condition (3-2). Moreover, every solution converging to a soliton satisfies this property:

Proposition A.1.7 (Exponential decay). Let $m=3$, or $m=2,4$ with $0<\lambda \leq \lambda_{0}$. Let $v=v(t)$ be $a$ $C\left(\mathbb{R}, H^{1}(\mathbb{R})\right)$ solution of (1-1) satisfying

$$
\lim _{t \rightarrow-\infty}\|v(t)-Q(\cdot-(1-\lambda) t)\|_{H^{1}(\mathbb{R})}=0 .
$$


Then there exist $K, \gamma, \varepsilon_{0}>0$ such that for every $t \leq-T_{\varepsilon}$ we have

$$
\|v(t)-Q(\cdot-(1-\lambda) t)\|_{H^{1}(\mathbb{R})} \leq K \varepsilon^{-1} e^{\gamma \varepsilon t} .
$$

Proof. Fix $\alpha>0$ small. Let $\varepsilon_{0}=\varepsilon_{0}(\alpha)>0$ be small enough such that for all $\varepsilon \leq \varepsilon_{0}$ and $t \leq-T_{\varepsilon}$

$$
\|v(t)-Q(\cdot-(1-\lambda) t)\|_{H^{1}(\mathbb{R})} \leq \alpha .
$$

Possibly choosing $\varepsilon_{0}$ even smaller, we can apply earlier arguments to the function $v(t)$ on the interval $\left(-\infty,-\frac{1}{2} T_{\varepsilon}\right]$ to obtain the desired result. We follow part (1) of Proposition A.1.3: Lemma A.1.4 holds for $z(t):=v(t)-Q\left(\cdot-(1-\lambda) t-\rho_{0}(t)\right)$ and $t \leq-\frac{1}{2} T_{\varepsilon}$, but now we have, by hypothesis,

$$
\lim _{t \rightarrow-\infty}\left|\rho_{0}(t)\right|+\|z(t)\|_{H^{1}(\mathbb{R})}=0 ;
$$

and therefore $\lim _{t \rightarrow-\infty} \mathscr{F}_{0}(t)=0$. (This can be made rigorous by taking a sequence $t_{n} \rightarrow-\infty$ large enough and such that $\left\|v\left(t_{n}\right)-Q\left(\cdot-(1-\lambda) t_{n}\right)\right\|_{H^{1}(\mathbb{R})} \leq \frac{1}{n}$. With this choice one has $\left|\rho_{0, n}\left(t_{n}\right)\right|+$ $\left\|z_{n}\left(t_{n}\right)\right\|_{H^{1}(\mathbb{R})} \rightarrow 0$, independently of $\varepsilon$. Rerunning as usual the proof in the interval $\left[t_{n}, t\right]$ and finally taking the limit $n \rightarrow+\infty$, we obtain the conclusion.) The rest of the proof is easy.

Note that monotonicity of mass was a key ingredient in this proof. This property apparently does not hold when $\lambda=0$ and $m=2,4$.

A.1.8. Uniqueness of the solution. Let $w(t):=v(t)-u(t)$. Then $w(t) \in H^{1}(\mathbb{R})$ and satisfies the equation

$$
\left\{\begin{array}{l}
w_{t}+\left(w_{x x}-\lambda w+a_{\varepsilon}\left[(u+w)^{m}-u^{m}\right]\right)_{x}=0 \quad \text { in } \mathbb{R}_{t} \times \mathbb{R}_{x} \\
\|w(t)\|_{H^{1}(\mathbb{R})} \leq K \varepsilon^{-1} e^{\gamma \varepsilon t} \quad \text { for all } t \leq-\frac{1}{2} T_{\varepsilon}
\end{array}\right.
$$

We must show that $w(t) \equiv 0$. Define the second-order functional

$$
\mathscr{F}_{0}(t):=\frac{1}{2} \int_{\mathbb{R}} w_{x}^{2}+\frac{1}{2} \int_{\mathbb{R}} w^{2}-\frac{1}{m+1} \int_{\mathbb{R}} a_{\varepsilon}(x)\left[(u+w)^{m+1}-u^{m+1}-(m+1) u^{m} w\right] .
$$

Reasoning as in the proof of Lemma 5.6, it is easy to verify the following properties:

(1) Lower bound. There exists $K>0$ such that for all $t \leq-\frac{1}{2} T_{\varepsilon}$,

$$
\mathscr{F}_{0}(t) \geq \frac{1}{2} \int_{\mathbb{R}}\left(w_{x}^{2}+w^{2}-m Q^{m-1} w^{2}\right)(t)-K \varepsilon^{-1} e^{\gamma \varepsilon t} \sup _{t^{\prime} \leq t}\left\|w\left(t^{\prime}\right)\right\|_{H^{1}(\mathbb{R})}^{2} .
$$

(2) Upper bound. There exists $K, \gamma>0$ such that

$$
\mathscr{F}_{0}(t) \leq K \varepsilon^{-2} e^{\gamma \varepsilon t} \sup _{t^{\prime} \leq t}\left\|w\left(t^{\prime}\right)\right\|_{H^{1}(\mathbb{R})}^{2} .
$$

However, this functional is not coercive, so in order to obtain a satisfactory lower bound, one has to modify the function $w$ in $\left(-\infty,-\frac{1}{2} T_{\varepsilon}\right]$ by setting

$$
\tilde{w}(t):=w(t)+b(t) Q^{\prime}(\cdot-t), \quad b(t):=\frac{\int_{\mathbb{R}} w(t) Q^{\prime}(\cdot-t)}{\int_{\mathbb{R}} Q^{\prime 2}},
$$

This modified function enjoys several properties:

(1) Orthogonality to the $Q^{\prime}$ direction: $\int_{\mathbb{R}} \tilde{w}(t) Q^{\prime}(\cdot-t)=0$. 
(2) Equivalence. There exists $C_{1}, C_{2}>0$ independent of $\varepsilon$ such that

$$
C_{1}\|w(t)\|_{H^{1}(\mathbb{R})} \leq\|\tilde{w}(t)\|_{H^{1}(\mathbb{R})}+|b(t)| \leq C_{2}\|w(t)\|_{H^{1}(\mathbb{R})}
$$

Moreover,

$$
\frac{1}{2} \int_{\mathbb{R}}\left(w_{x}^{2}+w^{2}-m Q^{m-1} w^{2}\right)(t)=\frac{1}{2} \int_{\mathbb{R}}\left(\tilde{w}_{x}^{2}+\tilde{w}^{2}-m Q^{m-1} \tilde{w}^{2}\right)(t)+O\left(e^{-\varepsilon \gamma|t|}\right) .
$$

(3) Control in the $Q$ direction:

$$
\left|\int_{\mathbb{R}} \tilde{w}(t) Q(\cdot-t)\right| \leq K \varepsilon^{-1} e^{\varepsilon \gamma t} \sup _{t^{\prime} \leq t}\left\|w\left(t^{\prime}\right)\right\|_{H^{1}(\mathbb{R})} .
$$

This property is proved similarly to the proof of (6-15): We use the fact that variation in time of the above quantity is of quadratic order on $\tilde{w}$.

(4) Coercivity. There exists $\lambda>0$ independent of $t$ such that

$$
\frac{1}{2} \int_{\mathbb{R}}\left(\tilde{w}_{x}^{2}+\tilde{w}^{2}-m Q^{m-1} \tilde{w}^{2}\right)(t) \geq \lambda\|\tilde{w}(t)\|_{H^{1}(\mathbb{R})}^{2}-K\left|\int_{\mathbb{R}} \tilde{w}(t) Q(\cdot-t)\right|^{2} .
$$

(5) Sharp control. From the equivalence between $w$ and $\tilde{w}$ and the coercivity property we obtain

$$
\|\tilde{w}(t)\|_{H^{1}(\mathbb{R})}+\varepsilon|b(t)| \leq K \varepsilon^{-2} e^{\varepsilon \gamma t / 2} \sup _{t^{\prime} \leq t}\left\|w\left(t^{\prime}\right)\right\|_{H^{1}(\mathbb{R})} .
$$

The bound on $b(t)$ is proved similarly to (6-14).

Finally, from (A-22) we obtain, for $\varepsilon$ small enough and $t \leq-\frac{1}{2} T_{\varepsilon}$,

$$
\|w(t)\|_{H^{1}(\mathbb{R})} \leq K \varepsilon^{-2} e^{\varepsilon \gamma t} \sup _{t^{\prime} \leq t}\left\|w\left(t^{\prime}\right)\right\|_{H^{1}(\mathbb{R})}<\frac{1}{2} \sup _{t^{\prime} \leq t}\left\|w\left(t^{\prime}\right)\right\|_{H^{1}(\mathbb{R})}
$$

This implies $w \equiv 0$, proving uniqueness.

A.2. Proof of Proposition 4.2. The proof is similar to that of [Martel and Merle 2009, Proposition 2.2] (see also and [Martel and Merle 2007, Appendix]). We start by writing the error term $S[\tilde{u}]$ of (4-9) as

$$
S[\tilde{u}]=I+I I+I I I,
$$

with

$$
\begin{aligned}
I & :=S[R] \\
I I & :=w_{t}+\left(w_{x x}-\lambda w+m a_{\varepsilon} R^{m-1} w\right)_{x} \\
I I I & :=\left(a_{\varepsilon}\left((R+w)^{m}-R^{m}-m R^{m-1} w\right)\right)_{x} .
\end{aligned}
$$

Recall that $m=2,3,4$.

Lemma A.2.1. We have

$$
I=\varepsilon F_{1}(\varepsilon t ; y)+\frac{\varepsilon^{2} a^{\prime \prime}}{2 \tilde{a}^{m}}\left(y^{2} Q_{c}^{m}\right)_{y}+\varepsilon^{3} f_{I}(\varepsilon t) F_{c}^{I}(y),
$$


where

$$
F_{1}(\varepsilon t ; y):=\frac{c^{\prime}}{\tilde{a}} \Lambda Q_{c}-\frac{\tilde{a}^{\prime}}{\tilde{a}^{2}}(c-\lambda) Q_{c}+\frac{a^{\prime}}{\tilde{a}^{m}}\left(y Q_{c}^{m}\right)_{y} \in \mathcal{Y},
$$

and $\left|f_{I}(\varepsilon t)\right| \leq K, F_{c}^{I} \in \mathcal{Y}$. Finally, for every $t \in\left[-T_{\varepsilon}, T_{\varepsilon}\right]$,

$$
\left\|\varepsilon^{3} f_{I}(\varepsilon t) F_{c}^{I}(y)\right\|_{H^{2}(\mathbb{R})} \leq K \varepsilon^{3} .
$$

Proof of Lemma A.2.1. Recall that $\tilde{a}:=a^{\frac{1}{m-1}}$ and

$$
R(t, x)=\frac{Q_{c(\varepsilon t)}(y)}{\tilde{a}(\varepsilon \rho(t))}, \quad y=x-\rho(t), \quad \partial_{t} \rho(t)=c(\varepsilon t)-\lambda .
$$

Thus

$I=R_{t}+\left(R_{x x}-\lambda R+a_{\varepsilon} R^{m}\right)_{x}=\frac{\varepsilon c^{\prime}}{\tilde{a}} \Lambda Q_{c}-\frac{c-\lambda}{\tilde{a}} Q_{c}^{\prime}-\varepsilon \frac{\tilde{a}^{\prime}(c-\lambda)}{\tilde{a}^{2}} Q_{c}+\frac{1}{\tilde{a}} Q_{c}^{(3)}-\frac{\lambda}{\tilde{a}} Q_{c}^{\prime}+\frac{1}{\tilde{a}^{m}}\left(a(\varepsilon x) Q_{c}^{m}\right)_{x}$.

A Taylor expansion gives

$$
\left(a(\varepsilon x) Q_{c}^{m}\right)_{x}=a(\varepsilon \rho)\left(Q_{c}^{m}\right)_{x}+\varepsilon a^{\prime}(\varepsilon \rho)\left(y Q_{c}^{m}\right)_{x}+\frac{1}{2} \varepsilon^{2} a^{\prime \prime}(\varepsilon \rho)\left(y^{2} Q_{c}^{m}\right)_{x}+O_{H^{2}(\mathbb{R})}\left(\varepsilon^{3}\right) .
$$

Therefore,

$$
\begin{aligned}
I=\frac{\varepsilon c^{\prime}}{\tilde{a}} \Lambda Q_{c}-\frac{(c-\lambda)}{\tilde{a}} Q_{c}^{\prime}-\frac{\varepsilon}{m-1} \frac{a^{\prime}(c-\lambda)}{\tilde{a}^{m}} Q_{c}+\frac{1}{\tilde{a}} Q_{c}^{(3)}-\frac{\lambda}{\tilde{a}} Q_{c}^{\prime} & +\frac{1}{\tilde{a}}\left(Q_{c}^{m}\right)^{\prime}+\frac{\varepsilon a^{\prime}}{\tilde{a}}\left(y Q_{c}^{m}\right)_{x} \\
& +\frac{\varepsilon^{2} a^{\prime \prime}}{2 \tilde{a}^{m}}\left(y^{2} Q_{c}^{m}\right)_{x}+\varepsilon^{3} f_{I}(\varepsilon t) F_{c}^{I}(y) \\
= & \frac{1}{\tilde{a}}\left(Q_{c}^{\prime \prime}-c Q_{c}+Q_{c}^{m}\right)^{\prime}+\frac{\varepsilon c^{\prime}}{\tilde{a}} \Lambda Q_{c}-\varepsilon \frac{\tilde{a}^{\prime}}{\tilde{a}^{2}}(c-\lambda) Q_{c}+\frac{\varepsilon a^{\prime}}{\tilde{a}^{m}}\left(y Q_{c}^{m}\right)_{y}+\frac{\varepsilon^{2} a^{\prime \prime}}{2 \tilde{a}^{m}}\left(y^{2} Q_{c}^{m}\right)_{y}+\varepsilon^{3} f_{I}(\varepsilon t) F_{c}^{I}(y) \\
= & \varepsilon\left(\frac{c^{\prime}}{\tilde{a}} \Lambda Q_{c}-\frac{\tilde{a}^{\prime}}{\tilde{a}^{2}}(c-\lambda) Q_{c}+\frac{a^{\prime}}{\tilde{a}^{m}}\left(y Q_{c}^{m}\right)_{y}\right)+\frac{\varepsilon^{2} a^{\prime \prime}}{2 \tilde{a}^{m}}\left(y^{2} Q_{c}^{m}\right)_{y}+\varepsilon^{3} f_{I}(\varepsilon t) F_{c}^{I}(y) .
\end{aligned}
$$

Moreover $\left|f_{I}(\varepsilon t)\right| \leq K, F_{c}^{I}(y) \in \mathcal{Y}$, and (A-28) is satisfied.

Lemma A.2.2. The quantity II is given by

$-\varepsilon\left(\mathscr{L} A_{c}\right)_{y}(\varepsilon t ; y)+\varepsilon^{2}\left(\left(A_{c}\right)_{t}+c^{\prime}(\varepsilon t) \Lambda A_{c}\right)(\varepsilon t ; y)+m \varepsilon^{2} \frac{a^{\prime}(\varepsilon \rho)}{a(\varepsilon \rho)}\left(y Q_{c}^{m-1}(y) A_{c}(\varepsilon t ; y)\right)_{y}+\varepsilon^{3} F_{c}^{I I}(\varepsilon t ; y)$.

with $F_{c}^{I I}(\varepsilon t ; \cdot) \in \mathcal{Y}$, uniformly in time. If, in addition, Property IP holds for $A_{c}$, then

$$
\left\|\varepsilon^{3} F_{c}^{I I}(\varepsilon t ; y)\right\|_{H^{2}(\mathbb{R})} \leq K \varepsilon^{3} e^{-\gamma \varepsilon|t|} .
$$

Proof. We compute

$$
\begin{aligned}
& I I=\varepsilon\left(A_{c}(\varepsilon t ; y)\right)_{t}+\varepsilon\left[\left(A_{c}\right)_{y y}(\varepsilon t ; y)-\lambda A_{c}(\varepsilon t ; y)+\frac{a_{\varepsilon}}{a(\varepsilon \rho)} m Q_{c}^{m-1}(y) A_{c}(\varepsilon t ; y)\right]_{x} \\
& =-\varepsilon\left(\mathscr{L} A_{c}\right)_{y}(\varepsilon t ; y)+\varepsilon^{2}\left(A_{c}\right)_{t}(\varepsilon t ; y)+\varepsilon^{2} c^{\prime}(\varepsilon t) \Lambda A_{c}(\varepsilon t, y) \\
& +m \varepsilon^{2} \frac{a^{\prime}(\varepsilon \rho)}{a(\varepsilon \rho)}\left(y Q_{c}^{m-1}(y) A_{c}(\varepsilon t ; y)\right)_{y}+\varepsilon^{3} F_{c}^{I I}(\varepsilon t ; y),
\end{aligned}
$$

where $F_{c}^{I I}(\varepsilon t ; y)=O\left(y^{2} Q_{c}^{m-1}(y) A_{c}(\varepsilon t ; y)\right)_{y} \in \mathcal{Y}$. Now (A-29) follows from Property IP. 
Lemma A.2.3. Suppose Property IP holds for $A_{c}$. Then we have

$$
I I I=\varepsilon^{3} a^{\prime}(\varepsilon x)\left[\varepsilon^{m-2} A_{c}^{m}(\varepsilon t ; y)+\tilde{F}_{c}^{I I I}(\varepsilon t ; y)\right]+\varepsilon^{2} a_{\varepsilon} G_{c}^{I I I}(\varepsilon t ; y),
$$

with $\tilde{F}_{c}^{I I I}(\varepsilon t ; \cdot), G_{c}^{I I I}(\varepsilon t ; \cdot) \in 9$, uniformly for every $t \in\left[-T_{\varepsilon}, T_{\varepsilon}\right]$. Moreover, we have the estimate

$$
\|I I I\|_{H^{2}(\mathbb{R})} \leq K \varepsilon^{2} e^{-\gamma \varepsilon|t|},
$$

for every $t \in\left[-T_{\varepsilon}, T_{\varepsilon}\right]$.

Proof. Define $\widehat{I I I}:=a_{\varepsilon}\left((R+w)^{m}-R^{m}-m R^{m-1} w\right)$.

Suppose first that $m=2$. Then $\widehat{I I I}=a_{\varepsilon} w^{2}=\varepsilon^{2} a_{\varepsilon} A_{c}^{2}$, and taking the derivative, $I I I=\varepsilon^{3} a^{\prime}(\varepsilon x) A_{c}^{2}+$ $\varepsilon^{2} a_{\varepsilon}\left(A_{c}^{2}\right)^{\prime}$. Here $\left(A_{c}^{2}\right)^{\prime} \in \mathscr{Y}$ because of Property IP.

Now suppose $m=3$. We have $\widehat{I I I}=\varepsilon^{2} a_{\varepsilon}\left(3 Q_{c} A_{c}^{2}+\varepsilon A_{c}^{3}\right)$. From this we get

$$
I I I=\varepsilon^{3} a^{\prime}(\varepsilon x)\left(3 Q_{c} A_{c}^{2}+\varepsilon A_{c}^{3}\right)+\varepsilon^{2} a_{\varepsilon}\left(3\left(Q_{c} A_{c}^{2}\right)^{\prime}+\varepsilon\left(A_{c}^{3}\right)^{\prime}\right) .
$$

Finally, for the case $m=4$,

$$
\begin{aligned}
I I I & =\left(a_{\varepsilon} \varepsilon^{2}\left(6 Q_{c}^{2} A_{c}^{2}+4 \varepsilon Q_{c} A_{c}^{3}+\varepsilon^{2} A_{c}^{4}\right)\right)_{x} \\
& =\varepsilon^{3} a^{\prime}(\varepsilon x)\left(6 Q_{c}^{2} A_{c}^{2}+4 \varepsilon^{2} Q_{c} A_{c}^{3}+\varepsilon^{2} A_{c}^{4}\right)+\varepsilon^{2} a_{\varepsilon}\left(6\left(Q_{c}^{2} A_{c}^{2}\right)^{\prime}+4 \varepsilon\left(Q_{c} A_{c}^{3}\right)^{\prime}+\varepsilon^{2}\left(A_{c}^{4}\right)^{\prime}\right) .
\end{aligned}
$$

Thus (A-30) holds in each case, assuming Property IP.

Now we collect the estimates from Lemmas A.2.1, A.2.2 and A.2.3. We finally get

$$
S[\tilde{u}]=I+I I+I I I=\varepsilon\left(F_{1}-\left(\mathscr{L} A_{c}\right)_{y}\right)(\varepsilon t ; y)+\varepsilon^{2}\left(\left(A_{c}\right)_{t}+c^{\prime}(\varepsilon t) \Lambda A_{c}\right)(\varepsilon t ; y)+O\left(\varepsilon^{2} e^{-\gamma \varepsilon|t|}\right),
$$

provided Property IP holds for $A_{c}$.

A.3. End of proof of Proposition 4.7. In this section we will show that for all $t \in\left[-T_{\varepsilon}, T_{\varepsilon}\right]$ (cf. (4-29))

$$
\|S[\tilde{u}](t)\|_{H^{2}(\mathbb{R})} \leq K \varepsilon^{\frac{3}{2}} e^{-\gamma \varepsilon|t|},
$$

where $\tilde{u}$ is the modified approximate solution defined in (4-26). We do this by writing a decomposition

$$
S[\tilde{u}]=I+\widetilde{I I}+\tilde{I I},
$$

similar to that in Section A.2 (see (A-23)-(A-26)). Lemma A.2.1 applies, so the term $I$ is given by (A-27) with no change. The term $\tilde{I I I}$ can be written as

$$
\tilde{I I I}=\varepsilon^{3} a^{\prime}(\varepsilon x)\left(\varepsilon^{m-2} \eta_{c}^{m} A_{c}^{m}(\varepsilon t ; y)+\tilde{F}_{c}^{I I I}(\varepsilon t ; y)\right)+\varepsilon^{2} a_{\varepsilon}\left(G_{c}^{I I I}(\varepsilon t ; y)+\varepsilon^{m-1}\left(\eta_{c}^{m}\right)^{\prime} A_{c}^{m}\right),
$$

with $\tilde{F}_{c}^{I I I}(\varepsilon t ; \cdot), G_{c}^{I I I}(\varepsilon t ; \cdot) \in \mathcal{Y}$, uniformly for every $t \in\left[-T_{\varepsilon}, T_{\varepsilon}\right]$. This is proved exactly like Lemma A.2.3, the only novelty being the appearance of the term

$$
\varepsilon^{m+1} a_{\varepsilon}\left(\eta_{c}^{m}\right)^{\prime} A_{c}^{m}, \quad \text { with } \quad\left\|\varepsilon^{m+1} a_{\varepsilon}\left(\eta_{c}^{m}\right)^{\prime} A_{c}^{m}\right\|_{H^{2}(\mathbb{R})} \leq K \varepsilon^{m+\frac{1}{2}} e^{-\gamma \varepsilon|t|}
$$

We thus get the estimate

$$
\|\tilde{I I I}\|_{H^{2}(\mathbb{R})} \leq K \varepsilon^{2} e^{-\gamma \varepsilon|t|} \quad \text { for all } t \in\left[-T_{\varepsilon}, T_{\varepsilon}\right]
$$


Finally, for the term $\widetilde{I I}$, we show that

$$
\widetilde{I I}=-\varepsilon \eta_{c}(y)\left(\mathscr{L} A_{c}\right)_{y}(\varepsilon t ; y)+O_{H^{2}(\mathbb{R})}\left(\varepsilon^{\frac{3}{2}} e^{-\gamma \varepsilon|t|}\right) .
$$

This is done along the lines of the proof of Lemma A.2.2, as follows. We have $\left(\varepsilon A_{\#}(\varepsilon t ; y)\right)_{t}$

$$
=-(c-\lambda) \varepsilon^{2} \eta_{\varepsilon}^{\prime} A_{c}(\varepsilon t ; y)-(c-\lambda) \varepsilon \eta_{\varepsilon}\left(A_{c}\right)_{y}(\varepsilon t ; y)+\varepsilon^{2} \eta_{\varepsilon}\left(A_{c}\right)_{t}(\varepsilon t ; y)+\varepsilon^{2} c^{\prime}(\varepsilon t) \eta_{\varepsilon} \Lambda A_{c}(\varepsilon t ; y) .
$$

We use Lemma 4.5 and (4-28) to estimate this expression, obtaining

$$
\left(\varepsilon A_{\#}(\varepsilon t ; y)\right)_{t}=-(c-\lambda) \varepsilon \eta_{\varepsilon}(y)\left(A_{c}\right)_{y}(\varepsilon t ; y)+O_{H^{2}(\mathbb{R})}\left(\varepsilon^{\frac{3}{2}} e^{-\gamma \varepsilon|t|}\right) .
$$

On the other hand,

$$
\begin{aligned}
& \varepsilon\left(\left(A_{\#}\right)_{x x}-\lambda A_{\#}+\frac{a_{\varepsilon}}{a(\varepsilon \rho)} m Q_{c}^{m-1}(y) A_{\#}\right)_{x} \\
&=\varepsilon\left(\eta_{\varepsilon}\left(\left(A_{c}\right)_{y y}-\lambda A_{c}+\frac{a_{\varepsilon}}{a(\varepsilon \rho)} m Q_{c}^{m-1}(y) A_{c}\right)+2 \varepsilon \eta_{\varepsilon}^{\prime}\left(A_{c}\right)_{y}+\varepsilon^{2} \eta_{\varepsilon}^{\prime \prime} A_{c}\right)_{x} \\
&=\varepsilon \eta_{\varepsilon}\left(\left(A_{c}\right)_{y y}-\lambda A_{c}+\frac{a_{\varepsilon}}{a(\varepsilon \rho)} m Q_{c}^{m-1}(y) A_{c}\right)_{x} \\
& \quad+\varepsilon^{2}\left(3 \eta_{\varepsilon}^{\prime}\left(A_{c}\right)_{y y}-\lambda \eta_{\varepsilon}^{\prime} A_{c}+a_{\varepsilon} m \eta_{\varepsilon}^{\prime} Q_{c}^{m-1} A_{c}+3 \varepsilon \eta_{\varepsilon}^{\prime \prime}\left(A_{c}\right)_{y}+\varepsilon^{2} \eta_{\varepsilon}^{(3)} A_{c}\right) \\
&=\varepsilon \eta_{\varepsilon}\left(\left(A_{c}\right)_{y y}-\lambda A_{c}+m Q_{c}^{m-1}(y) A_{c}\right)_{y}+\varepsilon^{2} \eta_{\varepsilon} m \frac{a^{\prime}(\varepsilon \rho)}{a(\varepsilon \rho)}\left(y Q_{c}^{m-1} A_{c}\right)_{y} \\
&+\varepsilon^{2}\left(3 \eta_{\varepsilon}^{\prime}\left(A_{c}\right)_{y y}-\lambda \eta_{\varepsilon}^{\prime} A_{c}+a_{\varepsilon} m \eta_{\varepsilon}^{\prime} Q_{c}^{m-1} A_{c}+3 \varepsilon \eta_{\varepsilon}^{\prime \prime}\left(A_{c}\right)_{y}+\varepsilon^{2} \eta_{\varepsilon}^{(3)} A_{c}\right)+O\left(\varepsilon^{3} \eta_{\varepsilon}\left(y^{2} Q_{c}^{m-1} A_{c}\right)_{y}\right) .
\end{aligned}
$$

We now use Lemma 4.5 and Property IP to get the estimates

$$
\begin{aligned}
m \varepsilon^{2}\left|\frac{a^{\prime}(\varepsilon \rho)}{a(\varepsilon \rho)}\right|\left\|\eta_{\varepsilon}\left(y Q_{c}^{m-1} A_{c}\right)_{y}\right\|_{H^{2}(\mathbb{R})} & \leq K \varepsilon^{2} e^{-\gamma \varepsilon|t|}, \\
\left\|O\left(\varepsilon^{3} \eta_{\varepsilon}\left(y^{2} Q_{c}^{m-1} A_{c}\right)_{y}\right)\right\|_{H^{2}(\mathbb{R})} & \leq K \varepsilon^{3} \\
\varepsilon^{4}\left\|\eta_{c}^{(3)} A_{c}\right\|_{H^{2}(\mathbb{R})} & \leq \varepsilon^{\frac{7}{2}} e^{-\gamma \varepsilon|t|} \\
\left\|\varepsilon^{2} \lambda \eta_{\varepsilon}^{\prime} A_{c}\right\|_{H^{2}(\mathbb{R})} & \leq K \lambda \varepsilon^{\frac{3}{2}} e^{-\gamma \varepsilon|t|} \\
\varepsilon^{2}\left\|3 \eta_{\varepsilon}^{\prime}\left(A_{c}\right)_{y y}+a_{\varepsilon} m \eta_{\varepsilon}^{\prime} Q_{c}^{m-1} A_{c}+3 \varepsilon \eta_{\varepsilon}^{\prime \prime}\left(A_{c}\right)_{y}\right\|_{H^{2}(\mathbb{R})} & \leq K \varepsilon^{2} e^{-\gamma \varepsilon|t|} .
\end{aligned}
$$

Therefore

$$
\begin{aligned}
\varepsilon\left(\left(A_{\#}\right)_{x x}-\lambda A_{\#}+\frac{a_{\varepsilon}}{a(\varepsilon \rho)} m Q_{c}^{m-1}(y) A_{\#}\right)_{x} \\
=\varepsilon \eta_{\varepsilon}\left(\left(A_{c}\right)_{y y}-\lambda A_{c}+m Q_{c}^{m-1}(y) A_{c}\right)_{y}+O_{H^{2}(\mathbb{R})}\left(\varepsilon^{2} e^{-\gamma \varepsilon|t|}+\varepsilon^{3}\right) .
\end{aligned}
$$

Now (A-33) follows from (A-34) and (A-35). 
We return to the global estimate on $S[\tilde{u}]$. From (A-27), (A-32), (A-33), and Lemma 4.5 we get

$$
\begin{aligned}
S[\tilde{u}] & =\varepsilon\left(F_{1}(\varepsilon t, y)-\eta_{c}(y)\left(\mathscr{L} A_{c}\right)_{y}\right)(\varepsilon t, y)+O_{H^{2}(\mathbb{R})}\left(\varepsilon^{\frac{3}{2}} e^{-\gamma \varepsilon|t|}\right) \\
& =\varepsilon\left(1-\eta_{c}(y)\right) F_{1}(\varepsilon t ; y)+O_{H^{2}(\mathbb{R})}\left(\varepsilon^{\frac{3}{2}} e^{-\gamma \varepsilon|t|}\right) .
\end{aligned}
$$

The final conclusion of this appendix is a straightforward consequence of the following fact: For every $t \in\left[-T_{\varepsilon}, T_{\varepsilon}\right]$ we have

$$
\left\|\varepsilon\left(1-\eta_{c}(y)\right) F_{1}(\varepsilon t ; y)\right\|_{H^{2}(\mathbb{R})} \leq K \varepsilon e^{-\frac{1}{\varepsilon}-\gamma \varepsilon|t|} \ll K \varepsilon^{10} .
$$

for $\varepsilon$ small enough. Indeed, note that $\operatorname{supp}\left(1-\eta_{c}(\cdot)\right) \subseteq\left(-\infty,-\frac{1}{\varepsilon}\right]$. From $(\mathrm{A}-27)$,

$$
\left|F_{1}(\varepsilon t ; y)\right| \leq K e^{-\gamma|y|-\gamma \varepsilon|t|} \text {. }
$$

Now the desired estimate follows directly.

A.4. Proof of Lemma 6.4. Our proof of the virial inequality (6-26) follows closely that of [Martel and Merle 2005, Lemma 2]. Take $t \in\left[t_{1}, T^{*}\right]$ and set $y:=x-\rho_{2}(t)$. We have

$$
\partial_{t} \int_{\mathbb{R}} z^{2} \psi_{A_{0}}(y)=2 \int_{\mathbb{R}} z z_{t} \psi_{A_{0}}(y)-\rho_{2}^{\prime}(t) \int_{\mathbb{R}} z^{2} \psi_{A_{0}}^{\prime}(y) .
$$

Substituting the value of $z_{t}$ given by (6-13), we can express the right-hand side as a sum of terms:

$$
\begin{aligned}
& 2 \int_{\mathbb{R}}\left(z \psi_{A_{0}}(y)\right)_{x}\left(z_{x x}-\lambda z+m Q_{c_{2}}^{m-1}(y) z\right) \\
& -\left(c_{2}(t)-\lambda\right) \int_{\mathbb{R}} z^{2} \psi_{A_{0}}^{\prime}(y)-2\left(c_{2}(t)-\lambda-\rho_{2}^{\prime}\right)(t) \int_{\mathbb{R}} z Q_{c_{2}}^{\prime} \psi_{A_{0}}(y) \\
& 2 \int_{\mathbb{R}}\left(z \psi_{A_{0}}(y)\right)_{x}\left[(R+z)^{m}-R^{m}-m R^{m-1} z\right] \\
& -2 c_{2}^{\prime}(t) \int_{\mathbb{R}} z \Lambda Q_{c_{2}} \psi_{A_{0}}(y)+\left(c_{2}-\lambda-\rho_{2}^{\prime}\right)(t) \int_{\mathbb{R}} z^{2} \psi_{A_{0}}^{\prime}(y) \\
& \int_{\mathbb{R}}\left(z \psi_{A_{0}}(y)\right)_{x}\left(a_{\varepsilon}-2\right)(R+z)^{m} .
\end{aligned}
$$

Following [Martel and Merle 2005] and using (6-14) and (6-15) it is easy to check that, for $A_{0}$ large enough and for some constants $\delta_{0}, \varepsilon_{0}$ small,

$$
|(\mathrm{A}-38)+(\mathrm{A}-39)| \leq \frac{\delta_{0}}{100} \int_{\mathbb{R}}\left(z_{x}^{2}+z^{2}\right)(t) e^{-\frac{1}{A_{0}}|y|} .
$$

Estimating (A-36) and (A-37) is done as for $B_{1}$ and $B_{2}$ in [Martel and Merle 2005, Appendix B]. We get

$$
(\mathrm{A}-36)+(\mathrm{A}-37) \leq-\frac{\delta_{0}}{10} \int_{\mathbb{R}}\left(z_{x}^{2}+z^{2}\right)(t) e^{-\frac{1}{A_{0}}|y|} .
$$

Finally, (A-40) can be estimated as follows. From (6-11) and (6-12) we have for $t \geq t_{1}$

$$
c_{2}(t)=c_{\infty}+O\left(\varepsilon^{1 / 2}\right), \quad \rho_{2}(t)=\left(c_{\infty}-\lambda\right) t+O\left(\varepsilon^{1 / 2}\left(t-t_{1}\right)\right),
$$


and then

$$
\frac{9}{10} c_{\infty} \leq c_{2}(t) \leq \frac{11}{10} c_{\infty} ; \quad \rho_{2}(t) \geq \frac{9}{10}\left(c_{\infty}-\lambda\right) t .
$$

On the other hand, we can write

$$
\begin{aligned}
(\mathrm{A}-40)= & \int_{\mathbb{R}}\left(z \psi_{A_{0}}\right)_{x}\left(a_{\varepsilon}-2\right)\left[(R+z)^{m}-z^{m}\right]+\int_{\mathbb{R}}\left(z \psi_{A_{0}}\right)_{x}\left(a_{\varepsilon}-2\right) z^{m} \\
= & \int_{\mathbb{R}}\left(\psi_{A_{0}}\right)_{x}\left(a_{\varepsilon}-2\right)\left[(R+z)^{m}-z^{m}\right] z+\int_{\mathbb{R}} \psi_{A_{0}}\left(a_{\varepsilon}-2\right)\left[(R+z)^{m}-z^{m}\right] z_{x} \\
& \quad+\frac{m}{m+1} \int_{\mathbb{R}}\left(\psi_{A_{0}}\right)_{x}\left(a_{\varepsilon}-2\right) z^{m+1}-\frac{\varepsilon}{m+1} \int_{\mathbb{R}} \psi_{A_{0}} a^{\prime}(\varepsilon x) z^{m+1} .
\end{aligned}
$$

Then, from (1-13), (6-25) and by using that $t \geq t_{1} \geq \frac{1}{2} T_{\varepsilon}$, we get

$$
\left|\int_{\mathbb{R}}\left(\psi_{A_{0}}\right)_{x}\left(a_{\varepsilon}-2\right)\left[(R+z)^{m}-z^{m}\right] z\right| \leq K A_{0} e^{-\varepsilon \rho_{2}(t) / A_{0}}\|z(t)\|_{H^{1}(\mathbb{R})} \leq K A_{0} e^{-\gamma \varepsilon t}\|z(t)\|_{H^{1}(\mathbb{R})}
$$

for some $\gamma=\gamma\left(A_{0}, c_{\infty}, \lambda\right)>0$ independent of $\varepsilon$ and $D_{0}$. (See (A-13) for a similar computation.) Similarly,

$$
\begin{aligned}
\left|\int_{\mathbb{R}} \psi_{A_{0}}\left(a_{\varepsilon}-2\right)\left((R+z)^{m}-z^{m}\right) z_{x}\right| & \leq K A_{0} e^{-\gamma \varepsilon t}\|z(t)\|_{H^{1}(\mathbb{R})}, \\
\left|\int_{\mathbb{R}}\left(\psi_{A_{0}}\right)_{x}\left(a_{\varepsilon}-2\right) z^{m+1}\right| & \leq K A_{0} e^{-\gamma \varepsilon t}\|z(t)\|_{H^{1}(\mathbb{R})}^{m+1} .
\end{aligned}
$$

Finally, from (6-24) and (A-41),

$$
\left|\varepsilon \int_{\mathbb{R}} \psi_{A_{0}}(y) a^{\prime}(\varepsilon x) z^{m+1}\right| \leq K A_{0} e^{-\gamma \varepsilon t}\|z(t)\|_{H^{1}(\mathbb{R})}^{m+1} .
$$

In conclusion, $(\mathrm{A}-40)=O\left(A_{0} e^{-\gamma \varepsilon t}\|z(t)\|_{H^{1}(\mathbb{R})}\right)$, for $\varepsilon$ small enough.

From (A-41) we obtain the second term in (6-26). Collecting the estimates above we conclude the proof.

A.5. Proof of Lemma 6.8. This is very similar to [Martel and Merle 2005, Lemma 3]. Recall that $\phi=\phi\left(\tilde{y}\left(x_{0}\right)\right)$, with $\tilde{y}\left(x_{0}\right)=x-\left(\rho_{2}\left(t_{0}\right)+\sigma\left(t-t_{0}\right)+x_{0}\right)$. Therefore

$$
\partial_{t} \int_{\mathbb{R}} u^{2} \phi=-\int_{\mathbb{R}}\left(3 u_{x}^{2}+(\sigma+\lambda) u^{2}-\frac{2 m a_{\varepsilon}}{m+1} u^{m+1}\right) \phi^{\prime}+\int_{\mathbb{R}} u^{2} \phi^{(3)}-\frac{2 \varepsilon}{m+1} \int_{\mathbb{R}} a^{\prime}(\varepsilon x) u^{m+1} \phi,
$$

and

$$
\begin{array}{r}
\partial_{t} \int_{\mathbb{R}}\left(u_{x}^{2}-\frac{2 a_{\varepsilon}(x)}{m+1} u^{m+1}\right) \phi=\int_{\mathbb{R}}\left(-\left(u_{x x}+a_{\varepsilon} u^{m}\right)^{2}-2 u_{x x}^{2}+2 m a_{\varepsilon} u_{x}^{2} u^{m-1}\right) \phi^{\prime}+\int_{\mathbb{R}} u_{x}^{2} \phi^{(3)} \\
-\sigma \int_{\mathbb{R}}\left(u_{x}^{2}-\frac{2 a_{\varepsilon}}{m+1} u^{m+1}\right) \phi^{\prime}-\frac{\varepsilon}{m+1} \int_{\mathbb{R}} a_{\varepsilon}^{\prime} u^{m+1} \phi^{\prime \prime}-\frac{\varepsilon^{2}}{m+1} \int_{\mathbb{R}} a_{\varepsilon}^{\prime \prime} u^{m+1} \phi^{\prime} ;
\end{array}
$$

see for example [Martel and Merle 2005, Appendix C]. The conclusion follows from the arguments in the same reference, after we estimate the single new different term. In particular, we have

$$
-\int_{\mathbb{R}}\left(3 u_{x}^{2}+(\sigma+\lambda) u^{2}-\frac{2 m a_{\varepsilon}(x)}{m+1} u^{m+1}\right) \phi^{\prime}+\int_{\mathbb{R}} u^{2} \phi^{(3)} \leq K e^{-\left(t_{0}-t\right) / 2 K_{0}} e^{-x_{0} / K_{0}} .
$$


Indeed, using that $1 / K_{0}^{2} \leq \sigma / 2$, we have (discarding the term with $\lambda$ )

$$
-\int_{\mathbb{R}}\left(3 u_{x}^{2}+\sigma u^{2}-\frac{2 m a_{\varepsilon}(x)}{m+1} u^{m+1}\right) \phi^{\prime}+\int_{\mathbb{R}} u^{2} \psi^{(3)} \leq-\int_{\mathbb{R}}\left(3 u_{x}^{2}+\frac{\sigma}{2} u^{2}-\frac{2 m a_{\varepsilon}(x)}{m+1} u^{m+1}\right) \phi^{\prime} .
$$

Now we estimate the nonlinear term. Let $R_{0}>0$, to be chosen later. Consider the region defined by $t \geq t_{1},\left|x-\rho_{2}(t)\right| \geq R_{0}$. In this region we have, from the stability and Morrey's embedding,

$$
|u(t, x)| \leq\|u(t)-R(t)\|_{L^{\infty}(\mathbb{R})}+R(t, x) \leq K \varepsilon^{1 / 2}+K e^{-\gamma R_{0}},
$$

with $\gamma>0$ a constant. Taking $0<\varepsilon \leq \varepsilon_{0}$ sufficiently small and $R_{0}$ large enough, we have $\left|m a_{\varepsilon}(x) u^{m-1}\right| \leq$ $\sigma / 4$ in the region considered. For the complementary region, $\left|x-\rho_{2}(t)\right| \leq R_{0}$, we see from (6-11) and the hypothesis $\sigma<\frac{1}{2}\left(1-\lambda_{0}\right)$ that

$$
\left|\tilde{y}\left(x_{0}\right)\right| \geq\left|\rho_{2}\left(t_{0}\right)-\rho_{2}(t)-\sigma\left(t_{0}-t\right)+x_{0}\right|-\left|x-\rho_{2}(t)\right| \geq \frac{1}{2} \sigma\left(t_{0}-t\right)+x_{0}-R_{0} .
$$

Thus $\left|\phi^{\prime}(\tilde{y})\right| \leq K e^{-\gamma\left(t_{0}-t\right) / K_{0}} e^{-x_{0} / K_{0}}$. Collecting the estimates above we obtain (A-43).

Now we claim that

$$
\left|\frac{2 \varepsilon}{m+1} \int_{\mathbb{R}} a^{\prime}(\varepsilon x) u^{m+1} \phi\right| \leq K e^{-\varepsilon \gamma T_{\varepsilon}} e^{-\varepsilon \gamma\left(t_{0}-t\right) / K_{0}} e^{-\gamma \varepsilon x_{0} / K_{0}} .
$$

Indeed, set $\tilde{x}(t):=\rho_{2}\left(t_{0}\right)+\sigma\left(t-t_{0}\right)+x_{0}$. Then from $\sigma<\frac{1}{2}\left(1-\lambda_{0}\right)$ and (6-11) we have

$$
\begin{aligned}
\tilde{x}(t) & =\rho_{2}\left(t_{0}\right)-\rho_{2}(t)-\sigma\left(t_{0}-t\right)+\left(x_{0}+\rho_{2}(t)\right) \\
& \geq \frac{1}{2} \sigma\left(t_{0}-t\right)+\rho_{2}\left(t_{0}\right)+x_{0} \geq \frac{1}{2} \sigma\left(t_{0}-t\right)+\frac{1}{2} T_{\varepsilon}+x_{0},
\end{aligned}
$$

and thus for $\varepsilon$ small,

$$
\begin{aligned}
\left|\frac{2 \varepsilon}{m+1} \int_{\mathbb{R}} a^{\prime}(\varepsilon x) u^{m+1} \phi\right| & \leq K \varepsilon \int_{-\infty}^{\tilde{x}} e^{-\varepsilon \gamma|x|} e^{(x-\tilde{x}) / K_{0}} d x+K \varepsilon \int_{\tilde{x}}^{\infty} e^{-\varepsilon \gamma x} \\
& \leq K \varepsilon e^{-\tilde{x} / K_{0}}+K e^{-\varepsilon \gamma \tilde{x}} \leq K e^{-\gamma \varepsilon T_{\varepsilon}} e^{-\gamma \varepsilon\left(t_{0}-t\right) / K_{0}} e^{-\gamma \varepsilon x_{0} / K_{0}} .
\end{aligned}
$$

This last estimate proves (A-45). Integrating between $t$ and $t_{0}$ we get (6-32).

Next, by following the same kind of calculations (see [Martel and Merle 2005]), we have

$$
\partial_{t} \int_{\mathbb{R}}\left(u_{x}^{2}+u^{2}-\frac{2 a_{\varepsilon}(x)}{m+1} u^{m+1}\right) \phi \leq K e^{-\gamma\left(t_{0}-t\right) / K_{0}} e^{-x_{0} / K_{0}}+K e^{-\gamma \varepsilon T_{\varepsilon}} e^{-\gamma \varepsilon\left(t_{0}-t\right) / K_{0}} e^{-\gamma \varepsilon x_{0} / K_{0}} .
$$

After integration we get (6-34).

Now we prove (6-33). The procedure is analogous to (6-32); the main differences are in (A-44) and (A-45). For the first case we have $\tilde{y}\left(-x_{0}\right)=x-\left(\rho_{2}\left(t_{0}\right)+\sigma\left(t-t_{0}\right)-x_{0}\right)$ satisfies

$$
|\tilde{y}| \geq\left|\rho_{2}(t)-\rho_{2}\left(t_{0}\right)-\sigma\left(t-t_{0}\right)+x_{0}\right|-\left|x-\rho_{2}(t)\right| \geq \frac{1}{2} \sigma\left(t-t_{0}\right)+x_{0}-R .
$$


From the hypothesis we have $\hat{x}(t):=\rho_{2}\left(t_{0}\right)+\sigma\left(t-t_{0}\right)-x_{0}>t_{1} \geq \frac{1}{2} T_{\varepsilon}$. Therefore (A-45) can be bounded as follows:

$$
\begin{aligned}
\left|\frac{2 \varepsilon}{m+1} \int_{\mathbb{R}} a^{\prime}(\varepsilon x) u^{m+1} \phi\right| & \leq K \varepsilon \int_{-\infty}^{\hat{x}} e^{-\varepsilon \gamma|x|} e^{(x-\hat{x}) / K_{0}} d x+K \varepsilon \int_{\hat{x}}^{\infty} e^{-\varepsilon \gamma x} \\
& \leq K \varepsilon e^{-\hat{x} / K_{0}}+K e^{-\varepsilon \gamma \hat{x}} k \leq K e^{-\gamma \varepsilon \rho_{2}\left(t_{0}\right)} e^{-\gamma \varepsilon\left(t-t_{0}\right) / K_{0}} e^{\gamma \varepsilon x_{0} / K_{0}} .
\end{aligned}
$$

Collecting the estimates above and integrating between $t_{0}$ and $t$, we obtain the conclusion.

A.6. Some identities related to the soliton $\boldsymbol{Q}$. The following identities can be found in [Martel and Merle 2007, Appendix C]. Recall that $Q_{c}:=c^{\frac{1}{m-1}} Q(\sqrt{c} x)$ denotes the scaled soliton $(m>1)$. Recall also that $\theta=\frac{1}{m-1}-\frac{1}{4}$.

(1) Energy.

$$
E_{1}[Q]=\frac{1}{2}\left(\lambda-\lambda_{0}\right) \int_{\mathbb{R}} Q^{2}=\left(\lambda-\lambda_{0}\right) M[Q], \quad \text { with } \lambda_{0}=\frac{5-m}{m+3}
$$

(2) Integrals.

$$
\begin{aligned}
& \int_{\mathbb{R}} Q_{c}=c^{\theta-\frac{1}{4}} \int_{\mathbb{R}} Q, \quad \int_{\mathbb{R}} Q_{c}^{2}=c^{2 \theta} \int_{\mathbb{R}} Q^{2}, \quad E_{1}\left[Q_{c}\right]=c^{2 \theta+1} E_{1}[Q], \\
& \int_{\mathbb{R}} Q_{c}^{m+1}=\frac{2(m+1) c^{2 \theta+1}}{m+3} \int_{\mathbb{R}} Q^{2}, \quad \int_{\mathbb{R}} \Lambda Q_{c} Q_{c}=\theta c^{2 \theta-1} \int_{\mathbb{R}} Q^{2} .
\end{aligned}
$$

\section{Acknowledgments}

. The author thanks Y. Martel and F. Merle for presenting this problem to him and for their continuing encouragement during the elaboration of this work. The author is also grateful of Gustavo Ponce for useful discussions.

\section{References}

[Asano 1974] N. Asano, "Wave propagation in non-uniform media", Progr. Theoret. Phys. Suppl. 55 (1974), 52-79. MR 57 $\# 18471$

[Benjamin 1972] T. B. Benjamin, "The stability of solitary waves", Proc. Roy. Soc. (London) Ser. A 328 (1972), 153-183. MR 49 \#3348

[Berestycki and Lions 1983] H. Berestycki and P.-L. Lions, "Nonlinear scalar field equations, I: Existence of a ground state", Arch. Rational Mech. Anal. 82:4 (1983), 313-345. MR 84h:35054a

[Bona et al. 1980] J. L. Bona, W. G. Pritchard, and L. R. Scott, "Solitary-wave interaction", Phys. Fluids 23 (1980), 438-441.

[Bona et al. 1987] J. L. Bona, P. E. Souganidis, and W. A. Strauss, "Stability and instability of solitary waves of Korteweg-de Vries type", Proc. Roy. Soc. London Ser. A 411:1841 (1987), 395-412. MR 88m:35128 Zbl 0648.76005

[Cazenave and Lions 1982] T. Cazenave and P.-L. Lions, "Orbital stability of standing waves for some nonlinear Schrödinger equations”, Comm. Math. Phys. 85:4 (1982), 549-561. MR 84i:81015

[Dejak and Jonsson 2006] S. I. Dejak and B. L. G. Jonsson, "Long-time dynamics of variable coefficient modified Korteweg-de Vries solitary waves", J. Math. Phys. 47:7 (2006), 072703, 16. MR 2007d:35233 Zbl 1112.35136

[Dejak and Sigal 2006] S. I. Dejak and I. M. Sigal, "Long-time dynamics of KdV solitary waves over a variable bottom", Comm. Pure Appl. Math. 59:6 (2006), 869-905. MR 2007a:35127 Zbl 1145.35096 
[Fermi et al. 1955] E. Fermi, J. Pasta, and S. Ulam, "Studies of nonlinear problems I", technical report LA1940, Los Alamos National Labs, 1955. reprinted as pp. 143-156 in Nonlinear Wave Motion, edited by A. C. Newell, Amer. Math. Soc., Providence, RI, 1974.

[Fernandez et al. 1979] J.-C. Fernandez, C. Froeschlé, and G. Reinisch, "Adiabatic perturbations of solitons and shock waves", Phys. Scripta 20:3-4 (1979), 545-551. MR 80k:76022 Zbl 1063.76530

[Fröhlich et al. 2004] J. Fröhlich, S. Gustafson, B. L. G. Jonsson, and I. M. Sigal, "Solitary wave dynamics in an external potential”, Comm. Math. Phys. 250:3 (2004), 613-642. MR 2005h:35320 Zbl 1075.35075

[Grimshaw 1979a] R. Grimshaw, "Slowly varying solitary waves. I. Korteweg - de Vries equation", Proc. Roy. Soc. London Ser. A 368:1734 (1979), 359-375. MR 80m:76023a

[Grimshaw 1979b] R. Grimshaw, "Slowly varying solitary waves. II. Nonlinear Schrödinger equation”, Proc. Roy. Soc. London Ser. A 368:1734 (1979), 377-388. MR 80m:76023b

[Grimshaw and Pudjaprasetya 2004] R. H. J. Grimshaw and S. R. Pudjaprasetya, "Generation of secondary solitary waves in the variable-coefficient Korteweg-de Vries equation”, Stud. Appl. Math. 112:3 (2004), 271-279. MR 2005a:35243 Zbl 1141.35447

[Holmer $\geq 2011]$ J. Holmer, "Dynamics of KdV solitons in the presence of a slowly varying potential", preprint. arXiv 1001.1583

[Holmer and Zworski 2008] J. Holmer and M. Zworski, "Soliton interaction with slowly varying potentials", Int. Math. Res. Not. 2008:10 (2008), Art. ID rnn026. MR 2009i:37183

[Holmer et al. 2007a] J. Holmer, J. Marzuola, and M. Zworski, "Fast soliton scattering by delta impurities", Comm. Math. Phys. 274:1 (2007), 187-216. MR 2008k:35445 Zbl 1126.35068

[Holmer et al. 2007b] J. Holmer, J. Marzuola, and M. Zworski, "Soliton splitting by external delta potentials", J. Nonlinear Sci. 17:4 (2007), 349-367. MR 2009d:35312 Zbl 1128.35384

[Jonsson et al. 2006] B. L. G. Jonsson, J. Fröhlich, S. Gustafson, and I. M. Sigal, "Long time motion of NLS solitary waves in a confining potential”, Ann. Henri Poincaré 7:4 (2006), 621-660. MR 2007f:35269 Zbl 1100.81019

[Kalisch and Bona 2000] H. Kalisch and J. L. Bona, "Models for internal waves in deep water", Discrete Contin. Dynam. Systems 6:1 (2000), 1-20. MR 2000j:76049 Zbl 1021.76006

[Karpman and Maslov 1977] V. I. Karpman and E. M. Maslov, "Perturbation theory for solitons", Ž. Èksper. Teoret. Fiz. 73:2 (1977), 281-291. In Russian; translated in Soviet Phys. JETP 46:2 (1977), 537-559. MR 58 \#29454

[Kaup and Newell 1978] D. J. . Kaup and A. C. Newell, 361 (1978), 413-446.

[Kenig et al. 1993] C. E. Kenig, G. Ponce, and L. Vega, "Well-posedness and scattering results for the generalized Korteweg-de Vries equation via the contraction principle", Comm. Pure Appl. Math. 46:4 (1993), 527-620. MR 94h:35229 Zbl 0808.35128

[Ko and Kuehl 1978] K. Ko and H. H. Kuehl, "Korteweg-de Vries soliton in a slowly varying medium”, Phys. Rev. Lett. 40:4 (1978), 233-236. MR 58 \#14579

[Korteweg and de Vries 1895] D. J. Korteweg and G. de Vries, "On the change of form of long waves advancing in a rectangular canal, and on a new type of stationary waves", Philos. Mag. Ser. 539 (1895), 422-443.

[Lochak 1984] P. Lochak, "On the adiabatic stability of solitons and the matching of conservation laws", J. Math. Phys. 25:8 (1984), 2472-2476. MR 85f:35184 Zbl 0548.35103

[Lochak and Meunier 1988] P. Lochak and C. Meunier, Multiphase averaging for classical systems, with applications to adiabatic theorems, Applied Mathematical Sciences 72, Springer, New York, 1988. MR 89k:58254 Zbl 0668.34044

[Martel 2005] Y. Martel, "Asymptotic $N$-soliton-like solutions of the subcritical and critical generalized Korteweg-de Vries equations", Amer. J. Math. 127:5 (2005), 1103-1140. MR 2007a:35128 Zbl 1090.35158

[Martel and Merle 2002a] Y. Martel and F. Merle, "Blow up in finite time and dynamics of blow up solutions for the $L^{2}$-critical generalized KdV equation", J. Amer. Math. Soc. 15:3 (2002), 617-664. MR 2003c:35142 Zbl 0996.35064

[Martel and Merle 2002b] Y. Martel and F. Merle, "Stability of blow-up profile and lower bounds for blow-up rate for the critical generalized KdV equation", Ann. of Math. (2) 155:1 (2002), 235-280. MR 2003e:35270 Zbl 1005.35081

[Martel and Merle 2005] Y. Martel and F. Merle, "Asymptotic stability of solitons of the subcritical gKdV equations revisited", Nonlinearity 18:1 (2005), 55-80. MR 2006i:35319 Zbl 1064.35171

[Martel and Merle 2007] Y. Martel and F. Merle, "Description of two soliton collision for the quartic gKdV equation", preprint, 2007. To appear in Ann. Math. arXiv 0709.2672 
[Martel and Merle 2008] Y. Martel and F. Merle, "Refined asymptotics around solitons for gKdV equations", Discrete Contin. Dyn. Syst. 20:2 (2008), 177-218. MR 2009c:35411 Zbl 1137.35062

[Martel and Merle 2009] Y. Martel and F. Merle, "Stability of two soliton collision for nonintegrable gKdV equations", Comm. Math. Phys. 286:1 (2009), 39-79. MR 2010h:35348 Zbl 1179.35291

[Martel and Merle 2010] Y. Martel and F. Merle, "Inelastic interaction of nearly equal solitons for the BBM equation", Discrete Contin. Dyn. Syst. 27:2 (2010), 487-532. MR 2011c:35516 Zbl 1188.35165

[Martel and Merle 2011] Y. Martel and F. Merle, "Inelastic interaction of nearly equal solitons for the quartic gKdV equation", Invent. Math. 183:3 (2011), 563-648. MR 2772088 Zbl 05876207

[Martel et al. 2002] Y. Martel, F. Merle, and T.-P. Tsai, "Stability and asymptotic stability in the energy space of the sum of $N$ solitons for subcritical gKdV equations", Comm. Math. Phys. 231:2 (2002), 347-373. MR 2003j:35280

[Martel et al. 2010] Y. Martel, F. Merle, and T. Mizumachi, "Description of the inelastic collision of two solitary waves for the BBM equation", Arch. Ration. Mech. Anal. 196:2 (2010), 517-574. MR 2011f:35304 Zbl 1200.35267

[Merle 2001] F. Merle, "Existence of blow-up solutions in the energy space for the critical generalized KdV equation", J. Amer. Math. Soc. 14:3 (2001), 555-578. MR 2002f:35193 Zbl 0970.35128

[Miura 1976] R. M. Miura, “The Korteweg-de Vries equation: a survey of results”, SIAM Rev. 18:3 (1976), 412-459. MR 53 \#8689 Zbl 0333.35021

[Mizumachi 2003] T. Mizumachi, "Weak interaction between solitary waves of the generalized KdV equations", SIAM J. Math. Anal. 35:4 (2003), 1042-1080. MR 2005b:35243 Zbl 1054.35083

[Muñoz 2010] C. Muñoz, "On the inelastic 2-soliton collision for gKdV equations with general nonlinearity”, Int. Math. Research Notices 2010 (2010), 1624-1719.

[Muñoz 2011] C. Muñoz, "Inelastic character of solitons of slowly varying gKdV equations", preprint, 2011. To appear in Comm. Math. Phys. arXiv 1107.5328

[Muñoz $\geq 2011$ a] C. Muñoz, "Dynamics of soliton-like solutions for slowly varying, generalized KdV equations: refraction versus reflection", SIAM J. Math. Anal. 44, 1-60.

[Muñoz $\geq 2011 b]$ C. Muñoz, "On the soliton dynamics under slowly varying medium for nonlinear Schrödinger equations", Math. Annalen. arXiv 1002.1295

[Newell 1985] A. C. Newell, Solitons in mathematics and physics, CBMS-NSF Regional Conference Series in Applied Mathematics 48, Society for Industrial and Applied Mathematics, Philadelphia, PA, 1985. MR 87h:35314 Zbl 0565.35003

[Pego and Weinstein 1994] R. L. Pego and M. I. Weinstein, "Asymptotic stability of solitary waves", Comm. Math. Phys. 164:2 (1994), 305-349. MR 95h:35209 Zbl 0805.35117

[Perelman 2009] G. Perelman, "A remark on soliton-potential interactions for nonlinear Schrödinger equations", Math. Res. Lett. 16:3 (2009), 477-486. MR 2010k:35468

[Shih 1980] L. Y. Shih, "Soliton-like interaction governed by the generalized Korteweg-de Vries equation”, Wave Motion 2:3 (1980), 197-206. MR 82e:76019

[Verhulst 2006] F. Verhulst, Nonlinear differential equations and dynamical systems, Springer, Berlin, 2006. MR 91b:34002 $\mathrm{Zbl} 0854.34002$

[Wright 1980] J. Wright, "Soliton production and solutions to perturbed Korteweg-de Vries equations", Phys. Rev. A 21:1 (1980), 335-339.

[Zabusky and Kruskal 1965] N. J. Zabusky and M. D. Kruskal, "Interaction of "solitons" in a collisionless plasma and recurrence of initial states", Phys. Rev. Lett 15 (1965), 240-243.

Received 22 Dec 2009. Revised 3 Jun 2010. Accepted 13 Jul 2010.

Claudio Muñoz C.: cmunoz@math.uchicago.edu

Departamento de Ingeniería Matemática DIM, Universidad de Chile, Blanco Encalada 2120, piso 5, Casilla 170-3, Correo 3, Santiago, Chile

Current address: Department of Mathematics, The University of Chicago, 5734 S. University Avenue, Chicago, IL 60637, United States

http://math.uchicago.edu/ cmunoz 


\section{Analysis \& PDE}

pjm.math.berkeley.edu/apde

EDITORS

EDITOR-IN-CHIEF

Maciej Zworski

University of California

Berkeley, USA

BOARD OF EDITORS

\begin{tabular}{|c|c|c|c|}
\hline Michael Aizenman & $\begin{array}{l}\text { Princeton University, USA } \\
\text { aizenman@math.princeton.edu }\end{array}$ & Nicolas Burq & $\begin{array}{l}\text { Université Paris-Sud 11, France } \\
\text { nicolas.burq@math.u-psud.fr }\end{array}$ \\
\hline Luis A. Caffarelli & $\begin{array}{l}\text { University of Texas, USA } \\
\text { caffarel@ math.utexas.edu }\end{array}$ & un-Yung Alice Chang & $\begin{array}{l}\text { Princeton University, USA } \\
\text { chang@math.princeton.edu }\end{array}$ \\
\hline Michael Christ & $\begin{array}{l}\text { University of California, Berkeley, USA } \\
\text { mchrist@math.berkeley.edu }\end{array}$ & Charles Fefferman & $\begin{array}{l}\text { Princeton University, USA } \\
\text { cf@math.princeton.edu }\end{array}$ \\
\hline Ursula Hamenstaedt & $\begin{array}{l}\text { Universität Bonn, Germany } \\
\text { ursula@math.uni-bonn.de }\end{array}$ & Nigel Higson & $\begin{array}{l}\text { Pennsylvania State Univesity, USA } \\
\text { higson@math.psu.edu }\end{array}$ \\
\hline Vaughan Jones & $\begin{array}{l}\text { University of California, Berkeley, USA } \\
\text { vfr@math.berkeley.edu }\end{array}$ & Herbert Koch & $\begin{array}{l}\text { Universität Bonn, Germany } \\
\text { koch@math.uni-bonn.de }\end{array}$ \\
\hline Izabella Laba & $\begin{array}{l}\text { University of British Columbia, Canada } \\
\text { ilaba@math.ubc.ca }\end{array}$ & Gilles Lebeau & $\begin{array}{l}\text { Université de Nice Sophia Antipolis, France } \\
\text { lebeau@unice.fr }\end{array}$ \\
\hline László Lempert & $\begin{array}{l}\text { Purdue University, USA } \\
\text { lempert@math.purdue.edu }\end{array}$ & Richard B. Melrose & $\begin{array}{l}\text { Massachussets Institute of Technology, USA } \\
\text { rbm@math.mit.edu }\end{array}$ \\
\hline Frank Merle & $\begin{array}{l}\text { Université de Cergy-Pontoise, France } \\
\text { Frank.Merle@u-cergy.fr }\end{array}$ & William Minicozzi II & $\begin{array}{l}\text { Johns Hopkins University, USA } \\
\text { minicozz@ math.jhu.edu }\end{array}$ \\
\hline Werner Müller & $\begin{array}{l}\text { Universität Bonn, Germany } \\
\text { mueller@math.uni-bonn.de }\end{array}$ & Yuval Peres & $\begin{array}{l}\text { University of California, Berkeley, USA } \\
\text { peres@stat.berkeley.edu }\end{array}$ \\
\hline Gilles Pisier & $\begin{array}{l}\text { Texas A\&M University, and Paris } 6 \\
\text { pisier@math.tamu.edu }\end{array}$ & Tristan Rivière & $\begin{array}{l}\text { ETH, Switzerland } \\
\text { riviere@ math.ethz.ch }\end{array}$ \\
\hline Igor Rodnianski & $\begin{array}{l}\text { Princeton University, USA } \\
\text { irod@math.princeton.edu }\end{array}$ & Wilhelm Schlag & $\begin{array}{l}\text { University of Chicago, USA } \\
\text { schlag@math.uchicago.edu }\end{array}$ \\
\hline Sylvia Serfaty & $\begin{array}{l}\text { New York University, USA } \\
\text { serfaty@ cims.nyu.edu }\end{array}$ & Yum-Tong Siu & $\begin{array}{l}\text { Harvard University, USA } \\
\text { siu@math.harvard.edu }\end{array}$ \\
\hline Terence Tao & $\begin{array}{l}\text { University of California, Los Angeles, USA } \\
\text { tao@math.ucla.edu }\end{array}$ & Michael E. Taylor & $\begin{array}{l}\text { Univ. of North Carolina, Chapel Hill, USA } \\
\text { met@math.unc.edu }\end{array}$ \\
\hline Gunther Uhlmann & $\begin{array}{l}\text { University of Washington, USA } \\
\text { gunther@math.washington.edu }\end{array}$ & András Vasy & $\begin{array}{l}\text { Stanford University, USA } \\
\text { andras@math.stanford.edu }\end{array}$ \\
\hline an Virgil Voiculescu & $\begin{array}{l}\text { University of California, Berkeley, USA } \\
\text { dvv@ math.berkeley.edu }\end{array}$ & Steven Zelditch & $\begin{array}{l}\text { Northwestern University, USA } \\
\text { zelditch@math.northwestern.edu }\end{array}$ \\
\hline
\end{tabular}

PRODUCTION

contact@msp.org

Silvio Levy, Scientific Editor

Sheila Newbery, Senior Production Editor

See inside back cover or pjm.math.berkeley.edu/apde for submission instructions.

The subscription price for 2011 is US \$120/year for the electronic version, and \$180/year for print and electronic. Subscriptions, requests for back issues from the last three years and changes of subscribers address should be sent to Mathematical Sciences Publishers, Department of Mathematics, University of California, Berkeley, CA 94720-3840, USA.

Analysis \& PDE, at Mathematical Sciences Publishers, Department of Mathematics, University of California, Berkeley, CA 94720-3840 is published continuously online. Periodical rate postage paid at Berkeley, CA 94704, and additional mailing offices.

APDE peer review and production are managed by EditFLOW ${ }^{\mathrm{TM}}$ from Mathematical Sciences Publishers.

PUBLISHED BY

mathematical sciences publishers

http://msp.org/

A NON-PROFIT CORPORATION

Typeset in IATEX

Copyright $(2011$ by Mathematical Sciences Publishers 


\section{ANALYSIS \& PDE}

\section{Volume $4 \quad$ No. $4 \quad 2011$}

The corona theorem for the Drury-Arveson Hardy space and other holomorphic Besov- 499 Sobolev spaces on the unit ball in $\mathbb{C}^{n}$

ŞERban Costea, Eric T. SAwyer and Brett D. Wick

Sobolev space estimates for a class of bilinear pseudodifferential operators lacking symbolic 551 calculus

FRÉDÉRIC BERNICOT and RODOLFO H. TORRES

Soliton dynamics for generalized $\mathrm{KdV}$ equations in a slowly varying medium

Claudio Muñoz C. 